\title{
Textes posthumes II : Philosophie continentale, de Nietzsche à Heidegger
}

Par ROBERT BRISART

Université Saint-Louis à Bruxelles, Université de Luxembourg

Résumé Le présent numéro reprend le texte de leçons sur la philosophie continentale que Robert Brisart (1953-2015) a présentées à l'Université du Luxembourg. L'enjeu de ces leçons est de montrer que les œuvres de Nietzsche et (comme l'a suggéré Rorty) du second Heidegger participent du même «tournant linguistique » de la philosophie contemporaine dont témoignent celles de Dewey, Wittgenstein, Quine, etc.

Mots-clefs Nietzsche, Heidegger, langage, tournant linguistique, ontologie, herméneutique.

\section{Introduction}

Nous devons à Richard Rorty la notion de «tournant linguistique », qu'il a reprise à Gustav Bergmann dans les années soixante, afin de caractériser ce qu'était devenue, selon lui, une des tendances majeures de la philosophie contemporaine. The Linguistic Turn est précisément le titre d'une anthologie qu'il publie en 1967 et où sont repris quelques textes fondamentaux du tournant amorcé par la philosophie analytique autour des années cinquante.

$\mathrm{Au}$ départ, ce que Bertrand Russell a appelé la "posture analytique » constituait une façon nouvelle de pratiquer la philosophie en affirmant que son travail conceptuel nécessitait une analyse du langage. C'est de manière logique que fut d'abord conçue cette analyse, sur la base des travaux initiateurs de Gottlob Frege et de Bertrand Russell lui-même dans ses Principia Mathematica de 1910. Dans l'esprit de Russell, cette analyse logique devait d'abord servir à débusquer les énoncés mal formés dont résultaient quantité de 
faux problèmes traditionnellement véhiculés par la philosophie, ceux portant, par exemple, sur des entités métaphysiques incongrues. Cette même analyse logique devait aussi servir à montrer les règles strictes suivant lesquelles des énoncés complexes doués de sens peuvent être construits à partir d'énoncés atomiques relatifs à des faits ou états de choses empiriques. Cette conception nouvelle de la philosophie est surtout illustrée et défendue par Ludwig Wittgenstein dans son Tractatus logico-philosophicus de 1921, où il soutient que la logique est l'essence du langage et que les problèmes philosophiques ne se posent que "parce que nous nous méprenons sur la logique de notre langage $»$.

Autour des années 50, l'analyse du langage va se tourner vers son usage courant et entraîner du même coup une remise en question de la conception logiciste de l'analyse qui avait prédominé jusqu'alors. Cette conception semblait désormais convenir davantage au langage scientifique, mais beaucoup moins au langage ordinaire. D'ailleurs, dans l'esprit de Russell ou de Carnap, les obscurités philosophiques étaient le plus souvent tributaires des confusions, des simplismes et des erreurs du langage ordinaire et c'est donc essentiellement celui-ci qu'il s'agissait d'épurer et de corriger par les moyens de la logique. Mais, aux yeux de plusieurs philosophes analytiques, c'était l'inverse qu'il s'agissait à présent de défendre : les simplismes philosophiques proviennent de la logique qui ne parvient pas à rendre compte de toutes les subtilités du langage ordinaire. Représentatifs de ce mouvement sont notamment Gilbert Ryle (1900-1976), John Austin (1911-1960), Peter Strawson (1919-2006) et Paul Grice (1913-1988). Ce mouvement est souvent appelé aussi École d'Oxford, puisque tous les noms cités sont ceux de philosophes qui y ont enseigné.

Prenons, par exemple, l'œuvre de John Austin. Pour lui, quasiment tout le travail philosophique consiste en une analyse du langage ordinaire. Autrement dit, Austin pensait qu'une enquête philosophique sur un problème quelconque, par exemple « le réel », exigeait d'abord de dresser un inventaire complet du matériau linguistique dont nous disposons dans la langue courante pour évoquer le problème en question. Il s'agissait donc de rassembler tous les mots et toutes les constructions linguistiques que l'utilisation ordinaire associe au problème, puis de vérifier avec la plus grande précision, dans quelles circonstances et avec quelles intentions nous utiliserions chacune des expressions inventoriées. Plutôt que de tenter de procurer des définitions savantes à une chose quelconque, il semblait donc à Austin beaucoup plus propice de s'en tenir à toutes les distinctions et les relations communes que notre langage ordinaire juge utiles de faire à propos de cette chose. Ces usages linguistiques sont souvent marqués par une longue histoire, au cours de 
laquelle ils n'ont fait que s'enrichir de nuances et de subtilités, et ont fait ainsi la preuve de leur utilité pratique pour cerner un problème. Il y a donc beaucoup plus à tirer de la pratique ordinaire du langage et du parcours des territoires conceptuels qu'elle a mis en œuvre que de tout ce que nous pourrions dire de quelque chose en y réfléchissant par nous-mêmes comme nous y a invité pourtant une certaine conception de la philosophie remontant à Descartes. À propos des faits de perception auxquels il s'est longuement attaché, Austin notait ainsi que

nos mots usuels sont beaucoup plus subtils dans leurs usages et marquent beaucoup plus de distinctions que les philosophes ne s'en rendent compte, et que ces faits relatifs à la perception (...) sont beaucoup plus diversifiés et compliqués qu'on n'avait l'habitude de le croire (Le langage de la perception, trad. P. Gochet, Vrin, 2007, p. 79-80).

Une des conséquences les plus directes du recours philosophique à la pratique du langage ordinaire fut de déboucher quasiment partout sur des controverses liées aux circonstances d'utilisation très différentes d'une expression linguistique. Le mot « sorcière », par exemple, désigne-t-il des femmes qui ont fait un pacte avec le diable, ou des femmes dont on pense qu'elles ont fait un pacte avec le diable, ou encore des femmes qui ont tel ou tel comportement, ou encore autre chose ? Austin était parfaitement conscient que l'analyse du langage ordinaire pouvait avoir des issues divergentes, mais c'est aussi cette situation que la philosophie devait assumer plutôt que de chercher à $<$ la $>$ dépasser. Autrement dit, la conclusion à laquelle menait tout droit la philosophie analytique du langage ordinaire est que si nos utilisations des mots ne concordent pas toujours, et s'accordent même assez rarement, c'est qu'elles sont liées à des circonstances d'utilisation très différentes et que ces différences de contexte correspondent à des schèmes conceptuels différents, c'est-à-dire à des façons différentes de classifier les choses et de les mettre en relation, finalement à des façons très différentes aussi de les voir.

Je voudrais mettre l'accent, dit Austin, sur les conséquences désastreuses qui se produisent en général lorsqu'on s'embarque dans l'explication de l'usage d'un mot sans prendre sérieusement en considération plus d'une mince fraction des contextes dans lesquels il est effectivement utilisé (Le langage de la perception, p. 171).

Ainsi, les différences dans la manière de décrire ce qui est vu proviennent-elles très souvent, non pas simplement de différences dans notre savoir, dans la finesse de nos facultés discriminantes, dans notre propension à nous exposer, 
ou dans notre intérêt pour tel ou tel aspect de la situation totale ; elles peuvent aussi provenir du fait que ce qui est vu est vu différemment, vu comme ceci plutôt que comme cela. Et parfois il n'y aura pas qu'une seule manière correcte de décrire ce qu'on voit, pour la bonne raison qu'il n'existe pas de manière correcte de le voir (Le langage de la perception, p. 191).

Ces conclusions étaient déjà aussi <celles> auxquelles avait abouti le «second» Wittgenstein dans ses Philosophical Investigations de 1953. Bien que n'appartenant pas à l'École d'Oxford, Wittgenstein s'est lui aussi détourné de l'analyse logique du langage, qu'il avait d'abord préconisée dans le Tractatus, pour amorcer, dès les années 30, le tournant vers une analyse du langage ordinaire où, selon lui, était cristallisé désormais tout ce dont doit s'occuper la philosophie. À la thèse emblématique du Tractatus selon laquelle le langage est tableau logique du monde (qui résume les deux affirmations : « le tableau logique du monde est la pensée » et «la pensée est la proposition douée de sens ») s'oppose à présent la thèse des jeux de langage :

Combien y a-t-il de sortes de phrases ? L'assertion, l'interrogation et l'ordre peut-être ? - Il y en a d'innombrables, il y a d'innombrables catégories d'emplois différents de ce que nous nommons « signes », « mots », « phrases ». et cette diversité n'est rien de fixe, rien de donné une fois pour toutes. Au contraire, de nouveaux types de langage, de nouveaux jeux de langage, pourrions-nous dire, voient le jour, tandis que d'autres vieillissent et tombent dans l'oubli... L'expression « jeu de langage » doit ici faire ressortir que parler un langage fait partie d'une activité, ou d'une forme de vie... Il est intéressant de comparer la diversité des outils du langage et de leur mode d'emploi, la diversité des catégories de mots et de phrases, à ce que les logiciens (y compris l'auteur du Tractatus logico-philosophicus) ont dit de la structure du langage (Recherches philosophiques, $\S 23$, trad. F. Dastur et alii, $<$ Paris, $>$ Gallimard, 2004, p. 39-40).

Le langage est donc désormais considéré comme une réalité indissolublement liée aux pratiques d'une communauté linguistique ou $<$ comme $>$ ce que Wittgenstein appelle une «forme de vie». Les mots, les phrases, les signes linguistiques en général, n'ont dès lors plus de statut indépendant par rapport à qui s'en sert, aux circonstances spatio-temporelles dans lesquelles ils sont utilisés, à la langue dans laquelle ils sont exprimés, etc. C'est justement tout ce que voulait mettre de côté une approche purement logique des propositions. Les signes linguistiques sont au contraire totalement relatifs à une façon de parler qui est elle-même dépendante du contexte pratique des locuteurs et de leur projet dans des circonstances données. Aussi, la seule façon de circonscrire le sens d'un mot ou d'une phrase est d'étudier les façons dont on 
les utilise dans des jeux de langage concrets. Parler de « jeux de langage » est donc désormais une façon de dire que le langage est un processus comparable à n'importe quel jeu de cartes, de balle ou d'échec : chacun a des règles que l'on peut tout aussi bien appeler sa grammaire. C'est elle qui détermine la valeur, le rôle et les attributions des constituants ou pièces du jeu, c'est elle qui détermine aussi ce que peuvent faire ou ne pas faire les joueurs, etc. Si on fait la comparaison avec le jeu d'échec, les mots ont, comme les pièces, un sens ou une valeur, mais qui n'est ni intrinsèque, ni absolue : ce sens dépend de leur relation possible aux autres pièces dans une partie donnée. La seule spécificité du langage serait que le sens des mots se réfère à quelque chose qu'ils servent à dire et que donc le sens se double de la référence. Mais les objets de référence sont eux-mêmes fonction de la grammaire d'un jeu de langage particulier, c'est lui qui, à propos des choses, prescrit de quelle sorte d'objets il s'agit, ce qu'en est l'essence, ce qu'il est possible ou pas d'en attendre, etc. Dans le Tractatus, l'intelligibilité du monde était réglée par l'architecture logique du langage ; dans les Philosophical Investigations, l'intelligibilité du monde reste certes encore relative au langage, mais le langage se diversifiant lui-même en une pluralité de jeux de langage, les ontologies se diversifient à raison des différentes façons de dire et de structurer le monde dans des pratiques et des contextes différents. La connaissance ontologique, celle qui demande de quels objets est fait le monde, devient donc une connaissance du langage ou du jeu de langage qui est utilisé pour constituer l'objectivité.

C'est en somme de tous les tenants de ce tournant linguistique de la philosophie que veut rendre compte Richard Rorty dans son anthologie de 1967, mais c'est surtout de ses aboutissants qu'il fait état dans un ouvrage célèbre paru en 1979 sous le titre Philosophy and the Mirror of Nature, traduit en français sous le titre L'homme spéculaire ${ }^{1}$. Telle qu'exposée dans cet ouvrage, la conséquence la plus directe du tournant linguistique serait l'ébranlement et même la fin des théories de la connaissance.

La gnoséologie ou la théorie de la connaissance a toujours considéré que la connaissance est miroir de la réalité et que, moins le miroir est déformant, plus la connaissance est vraie. Dans la tradition, cette conception a reçu plusieurs versions qui diffèrent seulement par les moyens mis en œuvre pour l'étayer : le recours à l'expérience chez les empiristes ou l'appel aux seules ressources de l'esprit chez les rationalistes. Selon Rorty, c'est le rêve d'une conformité au réel auquel vient mettre fin le tournant linguistique en brisant

${ }^{1}$ R. Rorty, Philosophy and the Mirror of Nature, Princeton, Princeton University Press, 1979 ; trad. fr. Th. Marchaisse, L’Homme spéculaire, Paris, Seuil, 1990. (N. de l'Éd.) 
l'image du miroir de la nature et en révoquant du même coup l'idée que le savoir puisse être reflet de la réalité ou quelque chose comme sa représentation plus ou moins adéquate. La fin des théories de la connaissance veut précisément dire cela : elle signifie la fin du rêve représentationaliste.

Mais pourquoi le tournant linguistique de la philosophie oblige-t-il précisément à se défaire du mythe représentationaliste ? Parce que l'approche nouvelle du langage - laquelle a fait suite à son analyse logique qui était encore de nature gnoséologique - a fini par montrer l'impossibilité d'une commensurabilité du savoir au réel. Nous l'avons vu, les objets ne sont rien d'autre que des productions de notre langage, des manières de procéder à l'ameublement sémantique du monde. Cela ne revient pas à nier la réalité, mais à soutenir en tout cas que nos objets, eux, ne se confondent pas avec elle; il ne sont que les référents que nous attribuons aux significations véhiculées par notre langage, dans le seul but de nous pourvoir de prises fiables dans le réel. $\mathrm{Au}$ demeurant, comme les langages sont pluriels et qu'il existe non pas un mais des jeux de langage fort différents entre eux, les systèmes d'objets le sont aussi et rien n'indique qu'il y aurait à nouveau quelque chose comme une garantie de commensurabilité entre eux. Comme le dit Rorty :

On admettra facilement que des questions comme «Est-ce que les Grecs se référaient à la "tempérance" lorsqu'ils employaient le mot sôphrosunè ? » et « Est-ce que les Nuers désignent l'âme par le terme kwoth », disparaissent dès que l'on tient compte du fait qu'il n'y a aucune raison spécifique de penser qu'à un mot donné d'une culture corresponde exactement un mot dans une tout autre culture. On admettra aussi qu'aucune paraphrase, aussi longue soit-elle, ne fasse ici l'affaire et qu'il faille se résoudre à plonger dans le bain d'un jeu de langage exotique. Mais dans le cas de la science, une telle attitude semble perverse. Dans ce cas-ci, nous sommes enclins à admettre qu'il y a bien quelque chose, là, à l'extérieur - le mouvement et ses lois par exemple -, quelque chose à quoi les gens entendaient se référer, ou à tout le moins auquel il se référaient sans en avoir clairement conscience. La recherche scientifique, croiton, est censée découvrir quelles sortes d'objets forment le monde et quelle sont leurs propriétés... (L'homme spéculaire, trad. Th. Marchaisse, Seuil, 1990, p. 297-298)

Ce que remet en question le tournant linguistique, c'est donc aussi et surtout cette forme la plus coriace du représentationalisme qui s'acharne à défendre l'idée de la vérité scientifique et, du même coup, instaure la distinction entre le discours scientifique et les autres discours plus confus. Contre quoi, Rorty reprend les arguments de Quine, qui sont aussi ceux de Kuhn et Feyerabend — philosophes que Rorty reconnaît tenir comme quelques-uns des héros de la 
philosophie issue du tournant linguistique. Selon la conception représentationaliste notamment défendue par l'empirisme logique du Cercle de Vienne, le sérieux du discours scientifique vient de ce contact avec la réalité que serait censé assurer le souci de fonder la science sur le donné d'expérience. Ce qui distinguerait alors la science d'Aristote de la science de Newton ce n'est pas de n'avoir pas eu le même référent, par exemple le mouvement, mais de lui avoir appliqué un traitement rationnel différent, plus maladroit dans le première que dans la seconde.

Cette idée repose en fait sur ce que Quine a appelé « les deux dogmes de l'empirisme ", que Rorty réinterprète comme suit. Le premier dogme repose sur l'idée qu'on peut faire la différence entre, d'une part, le référent et, d'autre part, ce que les gens en disent. Ce qui revient à prétendre qu'une même chose, comme le mouvement, dont on serait censé détenir l'essence était ce à quoi se référait Aristote en la nommant «déclivité du mouvement» et ce à quoi se référait Newton en la nommant «gravitation». Le second dogme de l'empirisme consiste, lui, à soutenir que si l'on peut ainsi traduire le langage d'Aristote dans celui de Newton, c'est que nous disposons toujours en principe d'un ensemble de descriptions faites dans un « langage d'observation neutre » permettant de cerner l'essence du référent et, sur cette base, d'examiner ensuite ce qui confirme ou infirme ce qui en est dit de part et d'autre. Mais c'est justement ce dont le tournant linguistique montre l'impossibilité : la correspondance avec le réel n'existe ni dans un cas, ni dans l'autre. Aristote et Newton se référaient à et parlaient de deux objets différents appartenant à deux schèmes conceptuels, deux jeux de langage, totalement différents. Entre eux, il n'y a pas de commensurabilité des significations et il n'y a donc pas non plus de traduction possible sur la base d'un même donné d'expérience neutre qui permettrait de se hisser au niveau d'une vérité absolue permettant de se prononcer à propos de la vérité ou de la fausseté des propositions de la science aristotélicienne et celles de la science newtonienne. La perception d'Aristote et donc ce que pouvait être à ses yeux un donné d'expérience, cela était sémantiquement structuré par son propre jeu de langage et donc aussi fort différent de ce que pouvait voir et identifier Newton. Ce qui ne veut pas dire non plus que la science de Newton ne soit pas préférable à celle de Aristote, mais, si tel est le cas, ce n'est certainement pas parce que les propositions du premier correspondent mieux à la réalité, mais simplement parce qu'elles nous permettent de mieux nous débrouiller dans l'existence.

D'où les deux conséquences de l'ébranlement des philosophies représentationalistes suscité par le tournant linguistique : le relativisme et le pragmatisme. Selon Rorty, la philosophie doit assumer de telles conséquences, pour autant qu'elle veuille renoncer à ses anciennes illusions. Le relativisme 
dit simplement le renoncement de toute prétention à une connaissance ou à une vérité objective et absolue, car il n'existe pas de connaissance ou de vérité indépendante du langage, de la culture et de la conceptualisation, bref indépendante d'un jeu de langage. Au huitième et dernier chapitre de Philosophy and the Mirror of Nature intitulé « Philosophy without Mirrors », Rorty qualifie la position qu'il défend de " philosophie édifiante » (edifying). Ce qui est simplement pour lui une façon de qualifier l'idéal d'une activité philosophique ouverte à la pluralité, aux différences, aux horizons sémantiques incommensurables, ouverture qui pourrait permettre aussi de réaménager l'horizon familier. Et il écrit à ce propos :

Pour un philosophe édifiant, l'idée même d'accéder à «toute Vérité » est absurde, parce que la notion (platonicienne) de vérité est absurde - et le reste quelle que soit la manière dont on l'entende: comme vérité du monde transcendante à toute description, ou comme vérité du monde accessible à travers une description si privilégiée qu'elle rend inutiles toutes les autres (parce qu'elles seraient toutes commensurables avec elle) (L'homme spéculaire, p. 415).

Mais ce renoncement à la Vérité n'est pas un renoncement à toute vérité. Il s'agit seulement d'écarter celle qui est objective et absolue, car, n'étant qu'un leurre fondé sur une prétendue conformité au monde, elle ne peut finalement rien nous apprendre du monde. Pour Rorty, il s'agit alors de lui préférer ces petites vérités simplement identifiées à « ce que nous avons de mieux à croire » (L'homme spéculaire, p. 342), parce que cela nous est beaucoup plus utile dans l'existence. C'est là précisément tout le sens d'une approche pragmatiste de la vérité.

Ce qui nous importe à présent est de constater que, dans Philosophy and the Mirror of Nature, Rorty mentionne les propres sources de sa conception d'une " philosophie édifiante » et il écrit à ce propos :

Les trois grands penseurs marginaux et édifiants de notre temps sont Dewey, Wittgenstein et Heidegger (L’homme spéculaire, p. 404).

La même chose se trouve déjà dite dans l'introduction :

Wittgenstein, Heidegger et Dewey, tels que je les conçois, sont des philosophes dont le but est d'édifier (L’homme spéculaire, p. 22).

Rorty se fait plus précis encore en écrivant : 
Wittgenstein, Heidegger et Dewey s'accordent à reconnaître que la notion de connaissance en tant que représentation adéquate rendue possible par certains processus mentaux et rendue intelligible par une théorie générale de la représentation, doit être abandonnée. Tous les trois rejettent la notion de " fondements de la connaissance » et la conception de la philosophie en tant qu'entreprise axée sur le problème cartésien : répondre au théoricien de la connaissance qui adopterait une position sceptique... Wittgenstein, Heidegger et Dewey nous ont fait entrer dans une période de philosophie « révolutionnaire $» . .$. en proposant de décrire l'ensemble des activités humaines à l'aide de nouvelles cartes, où ne figurent plus, tout simplement, les aspects qui paraissaient auparavant prédominants (L’homme spéculaire, p. 16-17).

Dans la postface à The Linguistic Turn, Rorty associe à nouveau les trois noms et écrit :

Les philosophes du vingtième siècle - Dewey, Heidegger et Wittgenstein avant tout, mais aussi Quine, Sellars et Davidson — nous ont montré comment nous soustraire au représentationalisme. Mais ils ne le firent pas en dissolvant de vieux problèmes, non pas en montrant qu'ils reposaient sur des « confusions conceptuelles » ou sur une "mécompréhension du langage », mais plutôt en suggérant une voie nouvelle pour décrire la connaissance et la recherche. Le seul sens où cette suggestion fut linguistique est le sens d'un changement comparable à celui qui fit passer de la cosmologie de Ptolémée et Aristote à celle de Copernic et Newton et ce fut un changement à propos du langage (The Linguistic Turn, The University of Chicago Press, 1967, p. 373).

De même, dans Philosophy and the Mirror of Nature, Rorty écrit toujours à propos des trois noms réunis :

Ils se sont acharnés à faire jouer l'argument holiste selon lequel les mots prennent leur sens au regard d'autres mots, et non en vertu de leur caractère représentatif, ainsi que son corollaire - à savoir que les discours tiennent leurs privilèges des hommes qui y ont recours et non de leur transparence au réel (L’homme spéculaire, p. 405).

Qu'afin de remonter aux sources du tournant linguistique, Rorty renvoie aux noms de Wittgenstein et de Dewey, on le comprend très aisément. En ce qui concerne Wittgenstein, nous l'avons évoqué, cela est parfaitement justifiable du fait même de l'introduction de la notion de « jeu de langage » dans les Philosophical Investigations. Et en ce qui concerne John Dewey, cela est également justifiable en regard de sa conception pragmatiste du langage. Fortement influencé par Darwin, John Dewey (1859-1952) considère l'esprit

9

Bull. anal. phén. XV 4 (2019)

https://popups.uliege.be/1782-2041/ @ 2019 ULiège BAP 
humain comme un instrument en perpétuelle évolution qui permet à l'homme de s'adapter à son milieu. Les productions de l'esprit, les pensées, et le langage qui les soutient, n'ont donc pas pour fonction la vérité comme conformité au réel, mais l'adaptation la meilleure possible de l'existence au réel. Le sens véritable de nos pensées ou de nos phrases ne se mesure ainsi qu'à l'aune de leurs conséquences pratiques relativement au contexte dans lequel nous les produisons. Autrement dit, au critère représentationaliste du sens, Dewey substitue le critère pragmatiste qui revient à affirmer qu'une pensée ou une phrase n'a de signification qu'en fonction des conséquences pratiques qu'entraîne son affirmation. Le satisfaisant se substitue ainsi au vrai et la division entre jugements satisfaisants et non satisfaisants ne peut être établie qu'en regard de ce qu'on vise à obtenir dans une situation donnée.

Par leur approche philosophique du sens et du langage, Dewey et le second Wittgenstein manifestent donc bel et bien un même souci de discréditer le mythe de la conformité au réel tel qu'il est postulé par la tradition philosophique. Dewey le fait en montrant que le critère du sens est davantage lié aux pratiques humaines qu'à une objectivité préalable. Ce que Wittgenstein transpose dans une théorie des jeux de langage où il montre que l'objectivité elle-même n'est que le résultat de la production linguistique des locuteurs et des interlocuteurs dans le contexte pratique qui est le leur. Ainsi, pour reprendre une expression de Rorty, Dewey et Wittgenstein sont assurément à la source d'une révolution en philosophie à laquelle on peut donner le nom de «tournant linguistique ». Toutefois, la question demeure de savoir ce qui autorise Rorty à associer à ces deux noms celui de Heidegger. Car si les positions philosophiques de ce dernier sont, de fait, assez proches d'une forme d'anti-représentationalisme, l'idée d'un tournant linguistique qui serait également à l'œuvre chez Heidegger est loin d'être évidente à première vue.

Le but de ce cours sera donc de montrer en quoi la thèse de Rorty est non seulement tout à fait plausible, mais qu'elle offre même un excellent angle d'attaque pour comprendre de quoi il est fondamentalement question dans la «question de l'être » ou dans la Seinsfrage qui est au cœur de toute la philosophie heideggerienne. En l'occurrence, il s'agira de montrer comment cette question a mené Heidegger à amorcer une tournant herméneutique de l'ontologie et pourquoi ce tournant herméneutique peut et même doit être compris comme un tournant linguistique. Ce qui, comme je tenterai de le montrer en guise de conclusion, ne devient véritablement explicite que chez Gadamer qui, tout en assumant l'héritage heideggerien, a forgé cette thèse dans son ouvrage majeur Wahrheit und Methode de 1960 : "L'être qui peut être compris est langage $»$. 
Cependant, comme le dit aussi Rorty, la philosophie de Heidegger est profondément post-nietzschéenne au sens fort du terme. Entendons par là qu'elle se situe dans un horizon ouvert par Nietzsche et que, en termes heideggeriens, on pourrait simplement appeler « la fin de la métaphysique ». Le thème de «la mort de Dieu » en est, dans le Zarathoustra, une allégorie bien connue, mais sa version plus sécularisée se trouve dans l'idée du « devenir fable du monde vrai ». Or pour Heidegger la fin de la métaphysique ne dit pas encore la sortie hors de la métaphysique, mais seulement sa conclusion. Et c'est pourquoi, selon Heidegger, la philosophie traditionnelle ou la métaphysique arrive à sa conclusion précisément chez Nietzsche où, du coup, elle s'y manifeste dans son essence la plus profonde. C'est aussi la raison pour laquelle la pensée de Nietzsche deviendra un des thèmes dominants de la pensée de Heidegger à l'époque qui fait suite à la publication de Sein und Zeit en 1927 et qui, vers les années 1935, verra s'amorcer ce que j'ai appelé le tournant herméneutique, lequel constitue la véritable sortie hors de la métaphysique entreprise par Heidegger.

Afin d'élucider la nature du tournant linguistique dans la pensée de Heidegger et son héritage chez Gadamer le cours se divisera donc en deux parties :

1. Nietzsche et la fin de la métaphysique

2. L'ontologie fondamentale de Heidegger et le tournant herméneutique $^{1}$

Sa conclusion portera sur le thème de l'être comme langage.

En outre, le cours tentera aussi de montrer en quoi la conception du langage propre au tournant linguistique de la "Continental Philosophy» inaugurée par Nietzsche et Heidegger est « absolutisée » sur le modèle exclusif de l'art et, de ce fait, reste fondamentalement différente de la conception «naturalisée » propre au pragmatisme d'un Quine ou d'un Davidson. Autrement dit, la question restera ouverte de savoir si l'on peut, comme tend à le faire Rorty, rapprocher l'issue de la philosophie analytique anglo-saxonne de l'issue de la Continental Philosophy sur la seule base du tournant linguistique amorcé des deux côtés.

${ }^{1}$ Le titre à la p. 51 porte «tournant linguistique » au lieu de «tournant herméneutique ». (N. de l’Éd.) 


\section{Première partie : Nietzsche et la fin de la métaphysique}

Les textes de Nietzsche seront cités d'après la traduction des Euvres philosophiques complètes parues chez Gallimard (citées OC suivi du numéro de tomaison) ${ }^{1}$.

Nous procéderons en quatre étapes. La première concernera « la métaphysique d'artiste », expression suivant laquelle Nietzsche a lui-même conçu au départ sa propre réaction et son alternative à la métaphysique traditionnelle. La seconde s'intitulera "la destruction de la métaphysique » et tentera de montrer comment Nietzsche a tenté de dépasser ses premières idées dans lesquelles il finira par déceler lui-même quelque chose comme un "relent d'hégélianisme ». La troisième étape concernera «la philosophie du Zarathoustra » où se trouvent contenues les grandes thèses de la philosophie originale de Nietzsche contre le nihilisme de la métaphysique traditionnelle. La quatrième étape sera consacrée à l'interprétation heideggérienne de Nietzsche et de son rapport à la métaphysique.

\section{La métaphysique d'artiste}

En 1873, Nietzsche publie le premier tome des Considérations inactuelles (Unzeitgemässe Betrachtungen). Il est intitulé David Strauss, l'adepte et l'écrivain (Der Bekenner und der Schriftsteller).

Qui est David Strauss ? Celui-ci est bien connu pour avoir publié, en 1835, une Vie de Jésus (Das Leben Jesu), ouvrage qui fit scandale à l'époque de sa parution. Strauss y montre que la doctrine de Hegel concernant le rapport entre philosophie et religion se heurte à une objection essentielle. Comment Hegel conçoit-il, en effet, ce rapport ? Réponse : en terme d'assimilation. Il suffit pour s'en convaincre de se rapporter au fameux texte Die Vernunft in der Geschichte (La raison dans l'histoire) qui est le contenu d'un cours de 1830.

\footnotetext{
${ }^{1}$ F. Nietzsche, Euvres philosophiques complètes, Paris, Gallimard, 1976 suiv. Les volumes cités par l'auteur sont les suivants : vol. I/1 : La Naissance de la tragédie suivi de Fragments posthumes (Automne 1869 - Printemps 1872) ; vol. I/2 : Écrits posthumes (1870-1873) ; vol. II/1 : Considérations inactuelles I et II suivi de Fragments posthumes (Été 1872 - Hiver 1873-1874) ; vol. II/2 : Considérations inactuelles III et IV suivi de Fragments posthumes (Début 1874 - Printemps 1876); vol. III/1: Humain, trop humain. Un livre pour esprits libres suivi de Fragments posthumes (1876-1878) ; vol. VI : Ainsi parlait Zarathoustra ; vol. VIII : Le Cas Wagner, Crépuscule des idoles, L'Antéchrist, Ecce homo, Nietzsche contre Wagner. (N. de l'Éd.)
} 
Dans la seconde partie de son introduction intitulée « La réalisation de l'Esprit dans l'histoire », Hegel termine en disant ceci :

La représentation la plus concrète de la Raison est Dieu... Dieu gouverne le monde et le contenu de son gouvernement, l'accomplissement de son plan, est l'histoire universelle. Saisir ce plan, voilà la tâche de la philosophie de l'histoire... La raison est l'intellection de l'œuvre divine... C'est dans la Raison que réside le Divin. Le contenu de la Raison est l'Idée divine. Considérée comme histoire universelle, la Raison n'est pas la volonté subjective, mais l'action de Dieu (La Raison dans l'histoire, trad. K. Papaioannou, <UGE, Paris, 1965,> p. 100-101).

La thèse de Hegel est donc que la réalisation de la raison humaine à travers l'histoire n'est rien d'autre que la réalisation du plan de Dieu ou de ce que Hegel nomme encore « l'Idée divine ». Celle-ci n'est pas extérieure à l'histoire où l'humanité se réalise elle-même, mais elle est au contraire immanente à cette histoire. Voilà ce que, selon Hegel, la philosophie a pour tâche de comprendre, et ce qu'elle expose alors conceptuellement est le même contenu que celui que la religion chrétienne expose métaphoriquement.

Strauss refuse précisément d'admettre cette assimilation du contenu de la philosophie à celui de la religion, et inversement. Selon lui, de réduire le contenu de la religion à des concepts philosophiques et donc de transposer ce contenu dans le registre de la rationalité, on ne fait qu'altérer la vérité même de la doctrine chrétienne. En effet, le contenu de cette religion, est fait d'une réalité historique que relatent les récits bibliques et évangéliques. Si l'on réduit le contenu de la religion à une simple métaphore, on perd de vue sa réalité historique pour la confondre avec quelque chose de purement symbolique.

Dans un premier temps, l'analyse de Strauss est donc de montrer qu'à force de vouloir mesurer le contenu de la foi chrétienne par rapport à des concepts dont il ne serait qu'un exposé métaphorique, on perd tout simplement l'authenticité de la religion. Contrairement à ce qu'enseigne Hegel, philosophie et religion ne sont donc pas de même essence : la philosophie est d'ordre conceptuel, tandis que la religion, du moins dans sa version chrétienne, est censée relater un événement réel ou historique.

Pourtant, les propos de Strauss sont trompeurs, car son but n'est pas simplement de rendre à la religion son autonomie par rapport à la philosophie. En fait, s'il les sépare l'une de l'autre, c'est afin de soumettre la religion à $<$ un> examen rationnel qui demande cette fois : qu'en est-il de la vérité de la religion, qu'en est-il de sa réalité et de sa prétendue historicité ? La question posée est donc de savoir si le contenu de la religion est historiquement avéré, ou si, en d'autres mots, l'historicité du Christ peut être considérée comme une 
vérité. Afin de répondre à ces questions, Strauss se propose de dégager des Évangiles la vraie figure du Christ et il aboutit à la conclusion que les Évangiles ne sont pas une biographie, mais la projection de conceptions messianiques héritées du judaïsme sur une personne, ayant du reste peut-être réellement existé. Le Christ historique, explique donc Strauss, n'est pas celui que l'on croit : il s'agit d'un homme ayant eu un destin tout ce qu'il y a de plus humain, mais dont le personnage historique a été transformé en mythe par les Évangiles. Ainsi, en montrant que l'authentique évaluation de la doctrine chrétienne revient à un examen historique, Strauss fait apparaître que cette doctrine n'est qu'un mythe qui exige qu'on passe cette fois à la philosophie pour atteindre la vérité ou le sens profond du mythe en question. Optant ainsi pour la philosophie par-delà la foi, Strauss montre enfin que ce que les Évangiles attribuent au Christ revient en fait à l'humanité tout entière : c'est elle qui est le fils unique de Dieu et c'est l'historicité humaine qui, seule, procure une image complète de Dieu.

On le voit, le livre de Strauss est surprenant à plus d'un égard. Tout d'abord, il semble porter une atteinte assez redoutable à la philosophie de Hegel, puisqu'il rompt l'identité entre la religion et la philosophie pour soutenir la thèse inverse : chacune a sa vérité propre et la vérité rationnelle de l'une et la vérité historique de l'autre ne sont pas la même chose, comme voudrait le faire croire la philosophie spéculative de Hegel. Mais, en voulant ainsi rendre sa propre vérité à la religion chrétienne, Strauss ne cherche en réalité qu'à l'ébranler en montrant que si le contenu de la religion est de nature historique, alors il faut la soumettre à une analyse philosophique. De celle-ci, il ressort que la religion n'est qu'un mythe dont la vérité revient en fin de compte à la philosophie, mais, cette fois, à une philosophie qui ne cherche plus à dire la même chose que la religion, mais qui veut en être le tribunal.

De la même façon, la position de Strauss vis-à-vis de Hegel apparaît tout aussi ambiguë. En effet, d'un côté, il rejette la thèse hégélienne de l'identité entre le contenu de la philosophie et le contenu de la religion, tandis que, d'un autre côté, il maintient cette identité dans la mesure où le jugement rendu par l'examen philosophique de la religion chrétienne est qu'elle n'est qu'un mythe qui cherche à refléter la vérité de l'humanité. Cette vérité est le sens de l'histoire humaine qui, à travers le progrès, ne cherche qu'à atteindre un seul but : la réalisation du ciel sur terre. L'idée, on le voit, reste très hégélienne, mais, cependant, avec une différence importante. Hegel considère, en effet, que la réalisation du ciel sur terre est accomplie par le savoir absolu réalisé dans sa propre philosophie, puisque, selon ses propres termes, le savoir absolu est la compréhension que son propre contenu équivaut à l'Idée ou au plan de Dieu. En d'autres termes, la philosophie de l'histoire de Hegel est une théorie 
eschatologique pour laquelle il n'y a pas de place pour un futur, puisque la raison peut se dire être parvenue à sa pleine réalisation dans le présent et donc prétendre à la fin de l'histoire.

La conception de l'histoire défendue par Strauss est radicalement opposée à cette forme d'eschatologie. Contre elle, Strauss affirme sa foi dans le futur et le progrès de l'histoire humaine plutôt que d'admettre que le contenu de la foi s'éclaire dans le présent de la philosophie. Il est intéressant de noter que cette position ambiguë à l'égard de la philosophie hégélienne de l'histoire est partagée par d'autres penseurs de l'époque qui appartiennent au mouvement dit des jeunes hégéliens et sur lesquels les idées de Strauss ont certainement eu une influence assez forte. Dans ce mouvement, les philosophes reconnaissent en général que le grand mérite de Hegel est d'avoir dégagé du passé les lois du développement historique. Son tort, en revanche, est d'avoir limité l'application de ces lois à l'explication du passé. Comme le dit Cieszkowski qui publie en 1838 à Berlin un petit ouvrage intitulé Prolegomena zur Historiosophie :

Dans son œuvre, Hegel n'a fait aucunement mention du futur estimant que la philosophie ne pouvait s'appliquer qu'à l'étude du passé et qu'elle devait laisser l'avenir complètement en dehors de sa spéculation. En ce qui nous concerne, nous prétendons au contraire que, sans la connaissance de l'avenir conçu comme partie intégrante de l'histoire et constituant la réalisation de la destinée humaine, il est impossible de parvenir à la connaissance de l'histoire universelle dans sa totalité spirituelle et dans son développement organique rationnel. Par conséquent, la mise en évidence d'une connaissance possible du futur est une condition préalable absolument indispensable si l'on veut donner au développement de l'histoire un caractère organique (trad. G. Debord, $<$ Prolégomènes à l'historiosophie, $>$ p. 15 ; modifiée d'après A. Cornu, $<$ Karl $>$ Marx< et Friedrich Engels. Leur vie et leur ouvre>, t. 1<: Les années d'enfance et de jeunesse. La gauche hégélienne (1818/1820-1844), Paris, Puf, 1955>, p. 142) ${ }^{1}$.

Le projet de Cieszkowski est donc clairement de refaire une lecture de l'histoire qui, tout en s'inspirant de Hegel, entend dépasser ses perspectives pour rendre au futur une place que ne peut lui reconnaître une philosophie qui, comme celle de Hegel, fait du présent un moment d'accomplissement et donc d'achèvement. Or, pour Cieszkowski, la connaissance possible du futur est en quelque sorte prescrite par la philosophie de Hegel. Celle-ci est en effet une

\footnotetext{
${ }^{1}$ La traduction française est due en réalité à Michel Jacob (Prolégomènes à l'historiosophie, Paris, Champ Libre, 1973). (N. d. l’Éd.)
} 
philosophie du concept et, comme telle, son mode est celui de la pure possibilité en attente d'une réalisation. Cette réalisation ne peut appartenir qu'au futur dont le mode est lui celui de l'actualisation ou de l'effectuation du concept, c'est-à-dire le devenir monde de la philosophie. Cette idée est éclairée par Cieszkowski, dès les premières lignes de son ouvrage :

L'humanité est enfin parvenue au stade de la conscience de soi... En revanche, ce qui touche à sa réalisation et à son achèvement reste encore à étudier, ainsi que l'élaboration du contenu intégral et la solution complète du problème dans son ensemble (p. 9-10 ; cf. également p. 112-113 et 116-117).

On l'entend bien, le projet est de restituer toute sa rigueur au thème hégélien de la raison agissant dans l'histoire, c'est-à-dire à la dialectique de Hegel, plutôt que de l'écarter. Il s'agit donc bien de dépasser le système de Hegel plutôt que de le rejeter. Quant à ce dépassement, il consiste à reprendre l'articulation hégélienne de l'histoire, mais en y intégrant le moment du moderne de façon à ne plus le considérer comme un point d'aboutissement, mais plutôt comme la transition nécessaire vers le futur. Bref, le présent de modernité cesse d'apparaître désormais comme une fin, mais doit se manifester comme la médiation entre le passé et le futur. Le présent est seulement l'achèvement du passé à travers la philosophie de Hegel. Celle-ci est le moment du savoir absolu où l'esprit se reconnaît comme le lieu d'unification de la raison et du réel. Mais cette unification n'appartient encore qu'à la pensée, elle est entièrement intériorisée par l'esprit, mais sans extériorisation hors du conceptuel. C'est donc dire que le présent n'est pas encore la pleine réalisation ou la concrétisation de l'esprit absolu. Celle-ci ne peut appartenir qu'à une troisième période qui s'ouvre devant nous, à savoir le futur. Quelle en sera l'essence ? Pour le jeunes hégéliens et notamment pour Cieszkowski, l'essence du futur est déjà connue : le futur ne pourra être que la synthèse concrète de la rationalité et de la réalité par-delà la synthèse seulement réalisée en esprit. Bref, la synthèse que le futur doit logiquement mener au jour ne sera plus seulement du ressort de la pensée mais de l'action. Aussi le futur ressemblera-t-il à cet âge où la tâche logiquement impartie à l'homme sera de transformer le monde de telle façon que la raison se réalise concrètement en lui.

Lorsqu'en 1872, près donc d'une quarantaine d'années après sa Vie de Jésus, David Strauss publie un ouvrage intitulé Der alte und der neue Glaube (L'ancienne et la nouvelle foi), c'est quelque chose d'assez proche de l'hégélianisme d'un Cieszkowski que l'on retrouve, mais agrémenté cette fois d'une 
idée nouvelle : la transformation du monde ou la concrétisation en lui de la raison, c'est la culture scientifique qui est en train de la réaliser.

La première partie de l'ouvrage a pour titre «Sommes-nous encore chrétiens ? » et la réponse est non. Reprenant la thèse essentielle de 1835 , Strauss écrit :

Le Jésus de l'histoire, de la science est un problème, et un problème ne peut être l'objet de la foi, l'idéal de la vie $<$ (D. Strauss, Der alte und der neue Glaube, Leipzig, Hirzel, 1872, p. 79)>.

Mais si la perspective chrétienne de la vie éternelle et toutes les autres consolations qui l'accompagnent sont donc aujourd'hui irrémédiablement perdues, cela signifie-t-il que nous ayons renoncé à la religion ? L'essence de la religion, répond Strauss, est à chercher dans le désir profondément humain d'être ce qu'il n'est pas et d'avoir ce qu'il n'a pas. Et c'est ce qu'il demande à son dieu : de le délivrer du joug de la nature et de sauvegarder face à elle sa liberté. Mais aujourd'hui, l'homme a découvert pour cela une voie nouvelle : celle générée par les découvertes de la science et les technologies. Ce sont les avancées de la connaissance rendues possibles par des savants comme Newton, Laplace ou Darwin, qui ont rendu cela possible. Certes, ce mouvement rencontre encore beaucoup d'obstacles qui en sont autant de limites. Mais c'est la preuve, dit Strauss, qu'il existe encore une place pour des souhaits et des prières. Ce n'est plus à d'anciennes chimères qu'elles sont aujourd'hui adressées, mais à une civilisation marquée par la culture scientifique et, par là, porteuse de la promesse d'un avenir toujours meilleur, d'un accroissement de l'équité sociale, de la garantie d'une paix mondiale, et d'une amélioration graduelle de la morale.

Toute la suite de l'ouvrage est alors consacrée à justifier cette idée à travers une examen de la façon dont cette culture nouvelle fondée sur la nouvelle science se manifeste dans notre façon de comprendre aujourd'hui le monde et dans la façon dont nous avons appris à ordonner notre propre vie en société. Par tout cela, Strauss entend montrer comment le nouveau croyant est finalement celui qui a décidé de faire de la terre son nouveau paradis.

Dans la première de ses Considérations inactuelles, Nietzsche n'a pas de mots assez durs pour critiquer la nouvelle religion proclamée par Strauss. La profession de foi faite par ce dernier n'est, selon les propres termes de Nietzsche, que l'œuvre d'un « philistin de la culture » (OC II/1, p. 24). D'après l'usage commun du terme, le philistin signifie l'homme inculte et le philistin qui se veut cultivé est précisément celui qui croit l'être sans l'être du tout. En effet, pour Nietzsche, Strauss confond partout la culture avec le culte de 
l'optimisme, il associe la culture à la confiance dans un progrès dont il croit deviner les signes rassurants dans la façon actuelle de vivre ou de penser, comme aussi dans toutes institutions du temps présent. Au fond, explique Nietzsche, il y a en cela une façon très bourgeoise de se féliciter de ce qu'on est et de comment on vit, tout en opinant du bonnet face aux productions du temps présent à la manière des Chinois qui ne cessent de courber l'échine. Mais, en raison même de cela, Strauss n'est au juste qu'un avatar de ce que Nietzsche appelle « la dégénérescence de $<$ la $>$ culture allemande ».

À propos de cette dégénérescence, Nietzsche s'est déjà expliqué dans une série de cinq conférences qu'il avait faites, un an auparavant, en 1872, devant la Société académique de Bâle. Rappelons que, à l'époque, Nietzsche a vingt-huit ans et qu'il occupe depuis trois ans la chaire de langue et de littérature grecque dans l'Université de la même ville. Dans cette série de conférences qui avaient pour titre : L'avenir de nos établissements d'enseignement, Nietzsche s'emploie à dénoncer le fait que, tel qu'il est conçu à cette époque, l'enseignement n'a plus d'autre finalité que de former les jeunes à devenir les serviteurs du présent et, dans ce but, à les imprégner de toutes les valeurs proposées par la modernité. Comme Nietzsche l'explique encore, ce culte du moderne n'est en fait que le culte du bonheur et de la réussite, et c'est pourquoi l'enseignement est seulement conçu dans le but que les jeunes gens puissent trouver leur place dans le présent, c'est-à-dire y trouver une profession et donc un travail.

« Nos établissements d'enseignement, dit Nietzsche... ne promettent que de former des fonctionnaires, des négociants, des officiers, des marchands, des agronomes, des médecins ou des techniciens ». À quoi il ajoute :

Toute éducation qui laisse apercevoir au terme de son itinéraire un poste de fonctionnaire ou un gagne-pain quelconque n'est pas une éducation pour la culture comme nous la comprenons (OC I/2, p. 132 ).

$\mathrm{Au}$ contraire, telle que l'entend Nietzsche, la culture ne doit servir qu'à contrarier, c'est-à-dire à forger un esprit critique qui permettra à ceux qui l'acquièrent d'être des esprits libres, capables de prendre leur existence en main. La vraie culture est celle qui doit permettre de faire face aux grands défis de l'existence, d'assumer tout ce que la vie possède de grandiose mais aussi de périlleux au lieu de chercher à l'éviter en préférant des valeurs qui allègent le poids de l'existence et procurent l'illusion d'une tranquillité facile. C'est pourquoi, conclut Nietzsche, 
la tâche suprême est d'abord de délivrer l'homme moderne de la malédiction du moderne... de la culture de ce temps présent (OC I/2, p. 131).

Il est assez évident que c'est la même idée d'une dégénérescence de la culture qui se trouve en filigrane de la critique acerbe que Nietzsche adresse à la «nouvelle religion » proposée par Strauss, puisqu'elle reflète tous les travers de la modernité et n'est au fond rien d'autre que «le catéchisme des idées modernes » $(O C \mathrm{II} / 1$, p. 33).. Mais, surtout, la nouvelle religion de Strauss trahit par ses relents le mal véritable qui se trouve être la cause de la dégénérescence de la culture. À propos du livre de Strauss, Nietzsche écrit :

Qu'une chose écrite en 1872, sente déjà le moisi en 1872, voilà qui me donne à penser $(O C \mathrm{II} / 1$, p. 37).

Sans doute, ne peut-on comprendre le sens de cette sentence si l'on ne fait pas le lien entre "l'odeur de moisi» que Nietzsche dit renifler chez Strauss et l'odeur cadavérique qu'il dira par ailleurs sentir s'exhaler de la philosophie de Hegel. En effet, même si Strauss ne cite pas Hegel à l'appui de sa nouvelle foi, Nietzsche voit, quant à lui, partout l'ombre de Hegel se profiler à travers le texte de Strauss. Il écrit à ce propos :

On voit qu'il a balbutié le hégélien, dans sa jeunesse, il lui est resté de ce temps une déformation (OC II/1, p. 77)

Et il ajoute par ailleurs :

Quand on a été une fois malade de hégélerie... on n'en guérit jamais complètement (OC II/1, p. 46).

Qu'est-ce donc que cette "la maladie hégélienne» que Nietzsche appelle encore l'« hégélerie »? Pour le dire d'un mot : il s'agit du culte de l'histoire. C'est ce culte que Nietzsche dit retrouver aux sources de la «nouvelle religion » de Strauss et c'est ce même culte qui, pour Nietzsche, se trouve aussi être la cause de la dégénérescence de la culture. Autrement dit, la culture dégénérée trouve ses racines dans la culture historique et celle-ci a précisément ses racines dans la philosophie de Hegel.

C'est ce que Nietzsche se propose de montrer dans la seconde des Considérations inactuelles qui paraît quelques mois après la première, en 1874. Elle a précisément pour titre «De l'utilité et des inconvénients des études historiques », et c'est ici que la critique de Hegel et des hégéliens devient radicale, comme le suggère le passage suivant : 
Je crois qu'il n'y a pas eu en ce siècle un seul tournant, un seul moment de faiblesse de la culture allemande qui n'ait été rendu plus dangereux encore par l'influence formidable et toujours vivace de cette philosophie, je veux dire de la philosophie hégélienne (OC II/1, p. 146).

Mais qu'est-ce qui justifie un jugement aussi sévère ? Nietzsche s'en explique à la suite de ces lignes. Selon lui, la philosophie de Hegel a fait de l'homme moderne un vieillard dont les occupations sont celles propres aux dernières heures de la vie : regarder en arrière, se souvenir, passer en revue toutes les choses du passé, bref s'adonner au culte de l'histoire. Pour Hegel, de toutes les heures de l'humanité, c'est précisément la dernière heure, celle de la vieillesse, qui est la plus importante. C'est à cet âge, en effet, que le regard peut enfin se tourner sur ce qui a été, sur tout le passé, et c'est donc aussi le moment où la conscience parvient à cet état d'achèvement où le sens de l'existence et de sa propre histoire se laisse enfin comprendre. Or, selon Hegel, cela est vrai aussi de l'humanité en général et de la vie de son esprit : son dernier moment, celui de son achèvement, est celui qui correspond à la compréhension du sens de toute l'histoire et c'est pourquoi ce moment est celui de la science en un sens absolu. Autrement dit, le savoir absolu n'est possible que lorsque, parvenant à son terme, la raison se comprend elle-même en comprenant que toute son histoire n'a été que le long processus menant au moment d'accomplissement où s'éclaire que ce processus n'a été que la marche progressive de la raison sur terre.

Mais, pour Nietzsche, il y a quelque chose de profondément désespéré en un tel savoir, puisque, se nourrissant uniquement du passé et donc entièrement inscrit dans la mémoire, il n'est concerné par aucun futur. Comme le dit Nietzsche, ce savoir est profondément stérile : la vie ne peut plus être devant lui, mais tout en arrière de lui. Elle appartient à une histoire passée et qui désormais se termine parce qu'elle est pleinement comprise. Aussi ce savoir ne peut-il servir à engendrer du nouveau ; il ne peut servir la vie.

Pourtant, bien que le savoir absolu ne soit en fait que «stérilité savante ", il affiche une suffisance et un orgueil sans limite, dans la mesure où il peut toujours se dire qu'il est l'accomplissement de l'histoire, comme si chaque marche qui a jalonné sa longue odyssée ne pouvait mener qu'à un but qui n'est autre que ce savoir absolu lui-même. Avec celui-ci se produit le moment où l'on peut se dire que le monde n'est plus à faire ou à inventer, mais qu'il n'y a pas lieu pour autant de s'en attrister, car le but est atteint par le présent du savoir. Selon Nietzsche, c'est aussi la raison pour laquelle la culture moderne qui se nourrit exclusivement du culte de l'histoire est une culture fondamentalement marquée par l'optimisme. Certes, des épigones comme 
Strauss et les Jeunes hégéliens veulent laisser les portes ouvertes à un futur que la philosophie de Hegel ne peut envisager. Mais, faisant cela, ils ne perçoivent le futur que comme la réalisation concrète du contenu encore abstrait de la science hégélienne. Pour eux, l'avenir n'a de sens que s'il se conforme à la rationalité hégélienne. Ce qui veut dire que l'avenir est déjà fixé par le présent de la philosophie et c'est pourquoi il ne saurait nous surprendre. À dire vrai, nous n'attendons rien d'autre du futur que ce que nous savons déjà de par sa configuration conceptuelle. C'est aussi pourquoi nous ne pouvons être qu'optimistes et, comme Strauss, ne considérer l'avenir que comme une extension de la raison à tous les domaines de la vie réelle, que ce soit sur le plan économique, social, politique, culturel et moral.

Ce que Nietzsche entend dénoncer, ce n'est pas seulement cet optimisme, mais c'est surtout le culte de l'histoire qui l'a généré. Il écrit :

Inactuelle, cette considération l'est encore parce que je cherche à comprendre comme un mal, un dommage, une carence, quelque chose dont l'époque se glorifie à juste titre, à savoir sa culture historique, parce que je pense même que nous sommes tous rongés de fièvre historienne et que nous devrions tout au moins nous en rendre compte (OC II/1, p. 94).

Comme l'explique Nietzsche, l'homme a certes besoin de l'histoire, car, contrairement aux animaux, il ne peut vivre « attaché au piquet du moment». Sa mémoire est d'abord une façon de se déjouer du néant qui s'attache à chaque moment qui, sitôt survenu, s'envole comme une page arrachée. C'est aussi de sa mémoire que l'homme détient le pouvoir de s'inspirer du passé, de soigner les blessures et les désillusions laissées par lui, de remplacer ce qui est perdu, et de refaire si possible en mieux ce qui s'est brisé. Bref c'est la mémoire qui permet à l'homme de transformer le passé en le mettant au service de la vie. Mais de trop s'en remettre au passé, les souvenirs deviennent écrasants. La mémoire finit alors par engourdir la puissance créatrice de l'homme, lui faisant perdre la force d'aspirer à quelque chose de nouveau. La mémoire doit donc aussi pouvoir faire place à la faculté d'oublier, car, dit Nietzsche, « toute action exige l'oubli » (OC II/1, p. 97). La vie a besoin de l'histoire, car c'est de cette manière qu'elle apprend à vouloir l'avenir avec toujours plus d'ardeur. Mais, pour passer du désir à l'acte, la vie a besoin aussi d'être anhistorique afin, cette fois, de n'avoir plus d'autre occupation que cet avenir lui-même. Tel est justement ce qu'on perd de vue lorsque l'histoire cesse d'être au service de la vie, pour ne plus se mettre qu'au service de la connaissance pure.

Ces constatations conduisent Nietzsche à envisager ainsi trois attitudes possibles à l'égard de l'histoire : l'attitude monumentale, l'attitude critique et 
l'attitude antiquaire. L'attitude monumentale est la façon dont l'homme se rapporte au passé, lorsqu'il est essentiellement tourné vers l'avenir et qu'il nourrit de grands projets. Cet homme actif a besoin de modèles, d'archétypes et d'exemples, qu'il cherche dans l'histoire et dans ce qui lui semble y constituer quelques rares moments sublimes et grandioses dont il fait autant de monuments. Toutefois, quand ce rapport monumental à l'histoire devient exclusif, la tentation est grande d'idéaliser certains âges d'or de l'humanité et de se dire que ce qui est grand a déjà existé et que cela a été tellement grand et inégalable qu'il n'est pas possible que cela se reproduise à nouveau. L'attitude monumentale finit alors par susciter le contraire de ses aspirations premières : elle cherchait de grands modèles pour l'avenir et elle sombre désormais dans la nostalgie d'âges révolus. L'idéalisation du passé finit alors par rendre l'homme actif purement réactif.

Pour que cette réactivité ne l'emporte pas, l'homme doit donc aussi être capable de briser les images anciennes. Contrairement à l'attitude monumentale, il ne s'agit plus ici de vouloir imiter, mais de traduire le passé devant le tribunal du présent et $<$ de $>$ le juger pour ses manques comme pour ses promesses non tenues. À cette condition seulement, l'histoire se met au service de la volonté d'avenir en révélant les aspects du passé susceptibles d'ouvrir des perspectives pour l'avenir. Telle est l'attitude critique, puisque sa relation au passé consiste en un examen critique seulement soucieux de créer un futur.

C'est bien sûr ce rapport à la vie et à l'avenir que méconnaît totalement l'attitude antiquaire. Ici, il n'est question que de se tourner vers le passé et d'en compiler tous les événements, car chacun d'eux semble éclairer l'homme sur sa propre situation présente. Il pourrait certes y avoir dans cette attitude une façon de mettre l'histoire au service de la vie, s'il ne s'agissait que de préserver un patrimoine pour le transmettre à ceux qui viendront. Mais très vite le souci de conservation prévaut ici sur le souci de transmission. "Ne rien oublier» devient la devise de cette attitude antiquaire pour laquelle l'homme semble n'avoir plus de chez soi que dans le passé. Le culte et la vénération de l'histoire n'est dès lors plus qu'une soif de collection et d'accumulation des vestiges du passé. L'action de l'homme n'est plus du tout motivée par la volonté d'engendrer du neuf, mais seulement motivée par le désir de conserver et de déambuler dans les musées qu'il s'est lui-même construits. La seule satisfaction qu'il puisse encore tirer de cela est seulement d'en être le conservateur.

La vie qui intéresse l'attitude antiquaire est une vie éteinte et tâcher de la conserver revient à immortaliser des momies. Comme le dit Nietzsche, « ici, l'homme s'enfouit dans la moisissure » (OC II/1, p. 112). Son savoir de l'histoire ne sert absolument plus la vie, il sert seulement la connaissance du 
passé, mais justement une connaissance qui, ayant perdu toute relation à la vie, n'a plus que les allures de « la savante misère » (OC II/1, p. 147). Wissendes Elend ou, ce qui revient au même " stérilité savante ", tels sont, nous l'avons $\mathrm{vu}$, les mots pour décrire la philosophie de Hegel qui, aux yeux de Nietzsche, représente très certainement la quintessence même de l'attitude antiquaire. Ce que cette philosophie nous propose n'est rien d'autre qu'une intériorisation du passé sous la forme de la remémoration. Pour dire cela, Hegel se sert d'un mot, dans la Phénoménologie de l'esprit : l'Er-innerung. Comme c'est en elle que consiste le savoir absolu, celui-ci n'est plus qu'un savoir purement encyclopédique qui a définitivement opté pour l'intériorité de la mémoire et qui, par là, refoule délibérément l'extériorité, c'est-à-dire au fond la vie concrète, au profit de la seule vie abstraite et représentationnelle de la pensée. Comme le dit Nietzsche :

Ce n'est plus la vie qui gouverne seule et tient en bride la connaissance du passé : toutes les bornes sont arrachées et l'homme est submergé par le flot de tout ce qui a jamais été. Toutes les perspectives sont prolongées à l'infini, aussi loin qu'il y eut un devenir. Nulle espèce n'a encore jamais vu se déployer à perte de vue un spectacle comparable à celui que nous présente l'histoire, cette science du devenir universel ; il est vrai qu'elle démontre en cela la dangereuse audace de sa devise : fiat veritas, pereat vita. Représentons-nous maintenant le processus spirituel qui se trouve ainsi déclenché dans l'âme de l'homme moderne. Le savoir historique, alimenté par des sources intarissables, l'inonde et l'envahit toujours davantage ; il est assailli de faits inconnus et incohérents, la mémoire ouvre toutes ses portes et n'est pas encore assez grande ouverte... L'homme moderne finit par avoir l'estomac chargé d'une masse énorme de connaissances indigestes qui se heurtent et s'entrechoquent dans son ventre. Ce bruit révèle la caractéristique la plus intime de cet homme moderne: la remarquable opposition entre une intériorité à laquelle ne correspond aucune extériorité et une extériorité à laquelle ne correspond aucune intériorité. Le savoir dont on se gave sans, le plus souvent, en éprouver la faim, parfois même malgré un besoin contraire, n'agit plus comme une force transformatrice orientée vers le dehors, il reste dissimulé dans une certaine intériorité chaotique, que l'homme moderne désigne avec une singulière fierté comme sa « profondeur » spécifique (Innerlichkeit)... L'intériorité éprouve peut-être alors cette sensation du serpent qui après avoir englouti quelques lapins vivants, s'étend tranquillement au soleil en évitant de bouger plus que nécessaire. Quiconque vient à passer par là ne désire qu'une chose, qu'une telle culture ne meure pas d'indigestion (OC II/1, p. 115-116).

Le renoncement à la conception d'une histoire ou à une connaissance du passé qui soit au service de la vie, la consécration d'une vie intérieure et d'un monde 
de représentation que plus rien ne relie à l'extériorité naturelle, l'affaiblissement d'une véritable volonté d'engendrement, l'exténuation de tout instinct créateur et novateur mais qui cache sa résignation sous les masques de l'autosatisfaction savante, de l'optimisme ou de la « nouvelle foi », voilà donc ce que sont, selon Nietzsche, les traits les plus saillants de la culture historique moderne qui justifient de parler de sa dégénérescence. Cet extrait peut sans doute servir à illustrer les conclusions de Nietzsche concernant la modernité :

L'homme moderne se tient fièrement au sommet de la pyramide du processus universel : en plaçant là-haut la clef de voûte de sa connaissance, il semble crier à la nature attentive : "Nous touchons au but, nous sommes le but, nous sommes la nature parvenue à son accomplissement ». Présomptueux Européen, trop orgueilleux, du dix-neuvième siècle, tu perds la tête! Ton savoir ne parachève pas la nature, il ne fait que tuer ta nature propre (OC II/1, p. 151).

On ne s'étonnera donc pas que la troisième partie des Considérations inactuelles, qui paraît en octobre 1874 - soit moins d'une dizaine de mois après la seconde -, soit consacrée à Arthur Schopenhauer et ait pour titre Schopenhauer l'éducateur (Schopenhauer der Erzieher). En effet, tout ce que Nietzsche considère comme les caractères essentiels de la culture de son temps se trouve justement combattu par la philosophie de Schopenhauer. Aux antipodes de Strauss, cette philosophie prône un pessimisme radical et, aux antipodes de Hegel, elle défend une conception anti-représentationaliste de la pensée visant à la rapprocher de la vie.

Schopenhauer (1788-1860) est l'auteur d'un ouvrage célèbre paru en 1818 sous le titre Le monde comme volonté et comme représentation. Dans une certaine mesure, l'ouvrage se présente comme un retour à Kant puisqu'il renoue avec la distinction radicale entre le réel et sa représentation, soit la distinction entre les choses telles qu'elles sont en elles-mêmes et les choses telles qu'elles nous apparaissent. Mais cette reprise de la dichotomie kantienne contre l'idéalisme hégélien n'a pourtant plus rien de kantien dans la façon dont Schopenhauer entend l'exploiter. Ce que montre, en effet, Schopenhauer, c'est que le monde de la représentation n'a aucune consistance. Il n'est qu'une illusion servant à cacher le monde réel à nous-mêmes; il <est $>$ un voile jeté sur la réalité afin de camoufler son insupportable vérité. En effet, le monde réel ou le monde de la vie ne ressemble pas aux représentations que nous nous en faisons. Ces représentations cherchent à le structurer en lui imprimant un ordre rationnel et sensé, alors que le monde réel est au contraire profondément sauvage et abscons. Mais qu'est ce qui autorise à attribuer à la réalité ces caractères qui la rendent incompatible avec le monde de notre représentation ? 
Le titre de l'ouvrage de 1818 suggère assez clairement la réponse : la réalité dans son en-soi est essentiellement marquée par la volonté. Mais de quelle volonté s'agit-il ? Elle n'est justement pas ce que nous en dit la représentation. Pour celle-ci, la volonté est toujours individuée, elle <est> un phénomène subjectif dirigé vers un objet. Parce qu'on se représente ainsi la volonté comme quelque chose de finalisé par un but qui est son objet, on peut également se la représenter comme ce qu'il peut y avoir de meilleur en l'homme, suivant précisément la spécificité de sa finalité, exactement comme le fait Kant. Mais, selon Schopenhauer, la volonté ne ressemble pas à sa représentation conceptuelle. Au lieu d'être individuée en un sujet, la volonté s'irradie partout de la même façon dans le monde en diluant ainsi les catégories d'espace et de temps. Partout et de tout temps, la volonté est l'éternel recommencement d'un même drame qui se produit sous des costumes différents. Pour Schopenhauer, cette volonté traduit simplement la vie. Elle n'est autre que le vouloir-vivre qui caractérise indistinctement tout étant et qui, en dépit des différenciations conceptuelles de notre représentation, fusionne tout en un seul et même moule. Ainsi la vie ressemble davantage à un tout dont les parties sont indistinctes dans la mesure où toutes sont marquées par la même volonté de vivre qui, comme telle, n'a ni sens, ni logique. Cette volonté est au contraire dévorante, désordonnée, pulsionnelle, aveugle et sans loi. C'est sous ces traits qu'elle caractérise la vie ou l'être en général comme ce qui, outre lui-même, n'a aucune finalité, ni donc aucune fin non plus, mais n'interrompt sa course éperdue qu'avec la survenue du non-être ou de la mort.

Aussi, à suivre Schopenhauer, faut-il bien admettre que la souffrance est l'essence de toute vie, puisqu'il n'existe aucun accomplissement, aucun assouvissement possible de la volonté. Par sa conscience, l'homme peut seulement déplorer la puissance volitive qui le dévore comme elle dévore tout ce qui est vivant, mais, au fond, il ne peut que très peu contre elle. Aussi s'efforcet-il d'engourdir et d'inhiber la volonté, et le monde de la représentation ne sert précisément qu'à cela. Ce monde illusoire est fait d'ordre, de classification, de raison et de logique et, comme tel, il est comparable à ce que la religion hindouiste appelle le « voile de Maya », car c'est lui que nous jetons sur la vie pour nous dérober, en même temps qu'elle, une tout <autre> vérité que celle que voudrait faire valoir notre représentation du monde. Mais, même sous les formes les plus sérieuses de la science rationnelle, cette représentation n'est encore qu'une illusion et, qui plus est, la manifestation la plus absurde de la vie elle-même, celle qui consiste à vouloir se dérober à soi-même.

Toujours selon Schopenhauer, seul l'art peut faire mieux que toutes les autres productions de l'homme. Certes l'art demeure, lui aussi, prisonnier de la volonté, puisqu'il est d'abord volonté d'œuvre et de beauté. Mais l'œuvre 
d'art, et principalement la musique, n'est pas seulement une illusion. Certes la musique est encore représentation, mais c'est précisément la vie qu'elle représente. Aussi, l'art musical nous rend-il pour la première fois spectateur de notre propre vie et de notre propre souffrance. Mettant la vie en scène, la musique nous met à distance de nous-mêmes et, nous détachant ainsi de notre propre existence, pour quelques moments elle nous la donne aussi à comprendre. C'est pourquoi, dans son langage universel, la musique nous charme et nous attriste : elle nous enchante car elle nous montre l'ardeur de la vie, mais nous attriste aussi car elle nous en fait percevoir la profonde souffrance. L'art musical, plus que tout autre, est donc la seule phénoménologie possible de la vie. Par là, cet art creuse un écart minimal entre nous-même et notre souffrance, mais qui suffit à produire une espèce de détachement ou de sublimation temporaire. Cette sublimation est donc aléatoire et précaire par rapport à ce que serait une sublimation complète que Schopenhauer finit par attribuer à une espèce d'état extatique auquel parviendrait seul celui qui, voulant ne plus rien vouloir, atteindrait ainsi au bien-être.

Considérons à présent les raisons pour lesquelles Nietzsche ne pouvait que se sentir attiré par la philosophie de Schopenhauer. La réponse est très certainement que cette philosophie recelait tous les racines de ce que pourrait être l'alternative à la culture moderne. Schopenhauer tente de montrer le caractère chimérique du monde de la représentation conceptuelle et, du même coup, dénonce les mensonges de l'idéalisme. Les soi-disant puissances de la rationalité sont confrontées à leur impuissance fondamentale à saisir la vie et, par là, l'esprit se trouve libéré pour endurer la vérité de son être et de l'être en tant que telle. En d'autres mots, il est amené à la contemplation tragique de la vie. Comme le dit Nietzsche :

L'homme de Schopenhauer prend sur lui la souffrance volontaire de la véracité (OC II/2, p. 47).

Mais, comme Schopenhauer le montre également, la vérité ne peut apparaître que lorsque nos yeux sont tournés vers la vie sans espérance, et c'est très exactement ce qui se passe avec l'art musical. Sa phénoménologie de la vie ne s'accompagne d'aucune tentative de la transcender. Autrement dit, s'il existe une seule vraie métaphysique, ce ne peut être qu'une «métaphysique d'artiste ».

C'est très certainement pour cette raison que Nietzsche considère Schopenhauer comme un "éducateur». Mais, cela en fait-il un disciple pour 
autant? Manifestement non. Car si Nietzsche a subi incontestablement l'influence de Schopenhauer, il s'en écarte aussi, comme le montre déjà son premier écrit important, La Naissance de la tragédie, écrit en 1872.

La thèse générale de cet ouvrage semble très proche des idées de Schopenhauer : le tragique est l'essence de la vie et ce n'est pas par la philosophie, mais par l'art, que l'homme est capable d'en saisir le sens. Plus spécifiquement, c'est dans l'art tragique ou dans la tragédie grecque que Nietzsche discerne l'expression la plus vive de la vie en ce qu'elle a de propre. Le jeu tragique est tramé de la même façon que la vie : il met en scène et manifeste le propre jeu ou le même combat que celui qui détermine la vie. Mais quelle est la nature de ce combat?

Pour Nietzsche, ce sont les deux composants essentiels de la tragédie qui, par leur opposition constante, servent à exprimer le sens de ce combat. Ces deux composants de la tragédie sont l'élément apollinien et l'élément dionysiaque.

Dans le panthéon olympien d'Homère, Apollon est le dieu de la lumière et de la beauté. Tout ce qui dans le monde est forme, fixité, finition, harmonie, mesure, cohérence et équilibre est une expression des attributs apolliniens. Mais Apollon est aussi le dieu du rêve, son œuvre enchante le monde, elle l'idéalise sous la forme d'un cosmos, mais cet enchantement n'est qu'une apparence, au double sens de ce qui apparaît mais n'est aussi qu'une illusion. Au contraire, Dionysos est une divinité pré-olympienne : il tire ses origines de l'ancien panthéon, du monde des Titans. Tout à l'inverse d'Apollon, c'est le dieu de l'obscurité et de la nuit, de la démesure, de l'incohérence et du chaos. Si Apollon est davantage rattaché aux œuvres plastiques et donc aux arts de la vison, Dionysos l'est au contraire à la musique, non pas à la musique qui ressemblerait à une belle architecture de sons, mais à celle qui, au contraire, incite à la danse, à l'excitation et à l'exubérance et à l'ivresse.

L'antagonisme nietzschéen entre l'apollinien et le dionysiaque fait immanquablement penser à l'antagonisme entre les deux mondes de Schopenhauer. Et de fait, l'élément apollinien circonscrit le domaine des formes individuées ou des phénomènes : ce domaine est donc celui de la représentation et, comme chez Schopenhauer, ce domaine est associé à celui du rêve et de l'illusion. Autrement dit, l'apollinien traduit le monde des apparences dans le double sens du terme. En revanche, le dionysiaque caractérise le domaine de ce que les Grecs appelaient l'apeiron, c'est-à-dire là où tout se confond dans un même fond chaotique, où tout ce qui était singularisé telle une forme et clairement séparé du reste, se trouve ramené à un même courant unique, à un même flux aveugle qui est le grand courant, le grand élan, de la vie. 
Pourtant, il existe sur ce point une différence assez importante entre Nietzsche et Schopenhauer. Elle tient au fait que, là où Schopenhauer ne voit qu'une opposition, Nietzsche voit au contraire une complémentarité. Pour Nietzsche, en effet, la vie n'est pas seulement réductible à l'élément dionysiaque. La vie est faite, au contraire, du combat fratricide que cette puissance mène constamment avec la puissance apollinienne. Vivant de ce combat, Apollon et Dionysos ne peuvent être l'un sans l'autre. Il y a dans leur discorde une profonde concorde qui les unit l'un à l'autre, comme le sont ceux qui s'affrontent dans un combat.

Comment comprendre le sens de cette complémentarité et comment comprendre qu'elle soit l'expression même de la vie ? Le dionysiaque, le fond obscur de la vie sans repos ne peut se révéler qu'en suscitant son propre contraire : l'apparence belle qui possède la stabilité et l'harmonie de la forme. Mais si la vie suscite les belles apparences, ce n'est jamais que pour en montrer l'illusion. Aussi est-ce en ébranlant les belles formes et en les rappelant à son fond obscur, que la vie se manifeste en sa propre démesure vertigineuse et terrifiante. Son propre fond dionysiaque, la vie ne le divulgue qu'à la faveur des belles apparences qu'elle finit toujours par faire vaciller. Inversement, concrétisation parfaite de la belle apparence apollinienne, le héros tragique ne sert qu'à laisser transparaître l'essence qui bat secrètement en lui. Cette essence est purement dionysiaque et, comme telle, elle finit toujours par happer le héros, le rappelant au fond de la vie comme la vague qui l'entraîne vers les abysses.

Pour Nietzsche, c'est précisément cela qu'est parvenue à exprimer la tragédie grecque et seulement elle. Couplant l'image à la musique, le rêve à l'ivresse, la forme au chaos, la lumière à la nuit, l'apparence à l'en-soi, en un mot, couplant l'apollinien au dionysiaque, la tragédie grecque fait apparaître le jeu qui préside à la vie même du monde.

Mais, à la faveur de sa réflexion sur la tragédie grecque, l'opposition de Nietzsche à Schopenhauer ne s'arrête pas au fait d'articuler le monde des apparences au monde en soi, au lieu de les séparer. Dans la mouvance, l'opposition de Nietzsche concerne aussi la fonction de l'art. Pour Schopenhauer, l'art, et plus spécifiquement l'art musical, était conçu comme un inhibiteur de la vie, une façon de s'en détacher en mettant en scène son fond de souffrance. Pour Nietzsche, l'art, et plus fondamentalement l'art tragique, n'est pas un dérivatif, mais une affirmation de la vie. En effet, malgré son fond de souffrance, la vie demeure attrayante précisément parce qu'elle ne peut se passer de formes apolliniennes, même si ce n'est que pour révéler, à travers elles, son fond obscur et tumultueux. Le vrai fond de la vie est certes dionysiaque, mais que serait-il sans les apparences apolliniennes qui, seules, 
lui permettent de se montrer? Aussi, le sentiment tragique est-il toujours lié à une exaltation de la vie que l'apollinisme rend non seulement supportable mais aussi enviable.

Schopenhauer en tant qu' « éducateur » n'aura dès lors servi qu'à administrer une leçon de pessimisme contre une forme d'optimisme uniquement fondée sur le monde de la représentation. Mais, une fois que ce monde de la représentation est réinterprété dans son rapport véritable à la vie, une nouvelle forme d'optimisme renaît à mesure que se précise l'opportunité du retour à une forme de culture en accordance avec la vie.

Comme on peut à présent le comprendre, la culture moderne à l'égard de laquelle Nietzsche est tellement critique, car il y voit un déni de la vie, est au fond une culture de l'apollinisme seul, soit une culture de la représentation qui, séparée de tout élément dionysiaque, ignore que la représentation n'est qu'un rêve, une illusion ou une apparence. Dans les deux premières Considérations inactuelles, Nietzsche nous a montré la culmination de cette culture purement apollinienne dans l'hégélianisme. Mais, dès La Naissance de la tragédie, Nietzsche avait déjà entrepris de montrer l'origine de cette culture appauvrie de son élément vital, et, selon lui cette origine est à chercher chez Socrate.

La thèse générale de Nietzsche est que l'avènement du socratisme marque la fin de l'esprit tragique grec. Plus précisément, c'est l'empreinte du socratisme sur Euripide, le dernier auteur tragique, qui signe justement le déclin de l'esprit qui animait l'œuvre de ses deux grands prédécesseurs, Eschyle et Sophocle. Dans les tragédies d'Euripide, on assiste, en effet, à la disparition de l'élément dionysiaque. Ce qui s'y trouve mis en scène n'est plus la vie elle-même, ce n'est plus ce combat permanent de l'ordre et du désordre, du juste et de l'injuste, de la raison et $<$ de $>$ la déraison, de la mesure et de la démesure. La raison cherche désormais à dépasser cette unité duelle qui lie les contraires. La raison veut la dominer, la dompter du regard et en tirer une morale. En ce qui concerne la conception de la tragédie, cela implique une exposition plus claire et plus didactique du drame. Le dialogue doit dès lors prévaloir sur la musique, tandis que la tension et l'incertitude sont atténuées par l'introduction du « prologue », sorte d'introduction au sujet qui résume et explique l'action qui va se dérouler. Quant à cette action tragique, elle ne sert plus qu'à offrir au spectateur une représentation plus nette et plus claire de luimême ; elle est conçue pour lui faire apparaître sa propre psychologie. Tel est justement le changement qui se produit dans les tragédies d'Euripide où la composition réaliste privilégie partout l'apollinisme.

Mais, montre Nietzsche, la tragédie elle-même ne peut survivre sans son élément dionysiaque, et, sans le dionysiaque, c'est l'élément apollinien qui 
perd la fonction qu'il remplissait dans la tragédie et prend un tout autre visage. Le plaisir des belles apparences fait désormais place à la rigueur d'une exposition réaliste des passions humaines. Or, selon Nietzsche, le suicide de la tragédie par injection de réalisme trouve sa véritable explication dans le fait qu'Euripide s'est soumis aux exigences d'un spectateur unique et idéal, à savoir Socrate.

À suivre Nietzsche, en effet, le socratisme n'est rien d'autre que l'avènement, dans la culture grecque, d'une mentalité nouvelle essentiellement marquée par la theoria, c'est-à-dire par l'aspiration à une vision rationnelle et logique du monde et des événements humains. Cette aspiration théorique n'est possible qu'en fonction du postulat qu'il existe une structure rationnelle de l'univers et que c'est à sa compréhension que l'esprit doit désormais appliquer tous ses efforts. Il est bien sûr, selon Nietzsche, qu'une telle idée était absolument incompatible avec la tension, l'ambiguité et l'incertitude que la tragédie considérait comme le fond même de la vie. Ainsi l'idéal de la philosophie grecque classique ne pouvait que rendre complètement obsolète l'esprit tragique. Tout ce qui, en dehors de l'élément apollinien, appartenait aussi à l'essence de la tragédie: l'exaltation, l'instinct, le pulsionnel, les affects et la sensualité, ne pouvait qu'être refoulé au nom de ce que la philosophie commençante tenait de son côté pour essentiel : la rigueur, la réflexion mûrie, l'esprit critique, le raisonnement, l'entendement, la représentation vraie, en un mot : la science. Plus qu'Euripide lui-même, ce sont Socrate et Platon qui, aux yeux de Nietzsche, ont signé l'arrêt de mort de l'esprit tragique. Mais, ce faisant, la philosophie classique est aussi née d'une négation de la vie. Socrate et Platon ont donc été les premiers nihilistes et tout ce qui a résulté de la tradition inaugurée par eux ne pouvait que porter les marques de la dégénérescence d'une culture qui n'était plus portée par la vie. Le propre idéal de cette culture exaltant «l'homme théorique », était lui-même marqué par la perte du goût pour la vie.

Dès lors, au $\S 17$ de La Naissance de la tragédie, Nietzsche pose le problème suivant :

La question qui nous occupe ici est de savoir si la puissance contre laquelle la tragédie s'est brisée a suffisamment de forces pour interdire à tout jamais dans l'art le retour de la tragédie et de la conception tragique du monde. Si ce qui a dévoyé l'ancienne tragédie, c'est l'impulsion dialectique au savoir et à l'optimisme scientifique, on pourrait conclure qu'il existe un éternel combat entre conception théorique et conception tragique du monde et qu'il ne sera possible d'espérer une renaissance de la tragédie que du jour où l'esprit scientifique aura atteint ses limites et, où la preuve en étant administrée, sa prétention à une validité universelle sera anéantie (La Naissance de la tragédie, OC I/1, p. 117). 
Or, selon Nietzsche, la démonstration des limites de la conception scientifique héritée du socratisme a d'abord été faite par Kant, et ensuite surtout par Schopenhauer. Le premier a montré l'impossibilité où se trouvait la représentation théorique de prétendre atteindre le monde tel qu'il est en luimême; le second a poussé les choses encore plus loin en montrant que le monde de la représentation n'était qu'un tissu d'illusions seulement conçues par l'homme pour se voiler à lui-même le fond tragique du monde et de la vie. Schopenhauer a dès lors introduit une conception nouvelle de l'art, et de la musique en particulier, qui, au lieu de se soumettre aux critères théoriques de l'esthétisme platonicien, a tenté de penser l'activité artistique en relation au monde de la vie. Comme le dit Nietzsche, il y a donc bien dans la philosophie allemande quelque chose comme une «sagesse dionysiaque mise en concepts » (OC I/1, p. 131).

Mais l'important est que, la preuve étant ainsi faite des impasses du modèle socratique de "l'homme théorique », une aurore nouvelle pouvait se lever pour la culture tragique. Celle-ci, estime Nietzsche, peut désormais renaître et le signe avant-coureur de cette renaissance est l'œuvre de Richard Wagner. Le remède à la dégénérescence de la culture moderne ne peut donc être autre que le renouveau de la culture tragique, et ce renouveau Nietzsche le voit émerger chez Wagner. D'où la topique de la quatrième des Considérations inactuelles qui parait en 1876 sous le titre « Richard Wagner à Bayreuth ». Allusion y <est> clairement faite ici au projet wagnérien du Festspielhaus que Nietzsche nomme alors le «théâtre de l'avenir », parce qu'il considère que l'opéra, tel que Wagner en conçoit alors la réalisation, constituera un art nouveau qui, renouant avec le mythe, dissociera d'abord le langage de son usage conceptuel pour lui rendre sa dimension poétique, et ensuite, réconciliant le mythe et la musique, réactivera l'union de l'apollinien et du dionysiaque pour mettre à nouveau l'art et la culture au service de la vie.

Peu importe ici la conception que Nietzsche se fait de l'opéra wagnérien. Cette conception a manifestement quelque chose de surfait et les espoirs que Nietzsche nourrit à l'égard de cet opéra seront du reste très vite déçus. Il est bien plus bien important de constater que si, à cette époque, Nietzsche place tant d'espoir dans le projet artistique de Wagner, c'est qu'il croit encore en une rédemption de la culture par l'art. Et, si tel est le cas, cela signifie que Nietzsche pense encore que la fonction de l'art tragique est d'exprimer ce qu'est véritablement la vie ou l'être en lui-même ? En d'autres mots, Nietzsche oppose l'art au langage conceptuel de la métaphysique classique, mais il ne dément pas pour autant la métaphysique comme telle. Au contraire, il croit encore en la possibilité pour un langage ou une expression humaine de s'élever jusqu'aux cimes de la vérité. Simplement, il conteste à la 
tradition socratique que la métaphysique soit l'apanage de l'homme théorique, mais il ne doute absolument pas qu'elle est celui de l'artiste. Aussi peut-on considérer qu'il reste, chez le jeune Nietzsche, quelque chose comme un élément socratique dans l'aspiration à une «métaphysique d'artiste ». C'est l'abandon de cette aspiration qui va bientôt le mener à une critique beaucoup plus radicale de toute prétention à se représenter le monde tel qu'il est. Cette critique touchera donc aussi la thèse que la vérité se tiendrait plutôt du côté de l'art, comme si, seul, ce dernier devait détenir le fin mot en ce qui concerne l'être, la vie ou le monde.

\section{La destruction de la métaphysique}

Penchons-nous à présent sur ces années qui font suite aux Considérations inactuelles au cours desquelles Nietzsche va entreprendre la destruction de toute métaphysique, en brisant du même coup son propre rêve d'une «métaphysique d'artiste ».

La déception profonde de Nietzsche à l'égard de Wagner ne tient qu'accessoirement à l'échec que fut la première représentation de $L$ 'Or $d u$ Rhin à Bayreuth en août 1876. Nietzsche, qui y assista, remarquera plus tard que, non seulement, le spectacle ne répondait pas à ses propres attentes philosophiques concernant une « culture de l'avenir », mais aussi que le public ne suivait pas, comme si, de toute façon, l'intérêt pour l'art avait désormais disparu. C'est surtout ce second point qui va nourrir sa réflexion durant les années qui suivent la publication des Considérations inactuelles. Elles le mèneront très vite à l'idée d'une insuffisance de la solution artistique au problème de la décadence culturelle moderne. Au fond, remarquera Nietzsche, l'art est inexorablement atteint d'une immaturité historique par rapport à la situation de l'homme moderne et c'est pourquoi l'art est aussi impuissant à guérir cet homme de son mal.

Telles sont les idées qui vont alimenter l'ouvrage que Nietzsche publie aux premiers jours de mai 1878 sous le titre Humain trop humain. La seconde partie de l'ouvrage paraîtra l'année suivante. L'orientation majeure en est que la métaphysique d'artiste relève encore d'une ultime illusion et qu'elle doit céder le pas à une science nouvelle, celle-là même que Nietzsche appellera un peu plus tard, en 1882, la Gaya Scienza, Le Gai savoir. Ce qui indique assez clairement un déplacement d'intérêt de l'art vers la science. Comment le comprendre? 
Un des leitmotive d'Humain trop humain est très certainement constitué par le thème de « l'art nécromant ». Cette idée apparaît au $§ 147$ de l'ouvrage où Nietzsche écrit ceci :

L'art assume accessoirement la tâche de conserver, et aussi de raviver çà et là certaines idées éteintes, décolorées ; il tresse, quand il s'acquitte de cette tâche, un lien enserrant diverses époques et il en fait revenir les esprits. Certes, c'est tout au plus une apparence de vie, comme au-dessus de tombeaux, qui s'élève ainsi ou, comme en rêve le retour des morts chéris, mais pour quelques instants au moins le sentiment ancien se ranime et le cœur se remet à battre à un rythme oublié le reste du temps (OC III/1, p. 120).

La thèse de Nietzsche sur l'art est donc désormais que, pour avoir encore aujourd'hui de l'effet sur l'esprit des gens, l'art ne peut que raviver des formes de l'esprit qui appartiennent définitivement au passé, parce qu'elles datent d'une époque où ces formes remplissaient les conditions pour que l'art soit vivant et efficace. Mais ces formes de l'esprit et ces conditions ont aujourd'hui disparu. Du même coup se sont aussi éclipsés toutes les idées et les sentiments dont l'art était porteur. Tout ce que l'artiste peut encore faire par son œuvre, c'est raviver des sentiments anciens, rappeler des idées révolues, mais à la façon dont on fait revenir les morts pour le plaisir éphémère de les revoir en rêve. C'est pourquoi, dit Nietzsche :

De même que, vieillard, on se rappelle sa jeunesse et célèbre les fêtes du souvenir, l'art ne sera plus bientôt pour l'humanité qu'un souvenir émouvant des joies de sa jeunesse. Jamais auparavant l'art n'a été compris peut-être avec autant de profondeur et d'âme que de nos jours où il semble que la magie de la mort le baigne de son halo (OC III/1, p. 156).

Autant dire que l'action de l'art est aujourd'hui rétrograde. L'art servait naguère à exprimer des vérités dans un monde où l'esprit était fait pour les entendre. Dans ce monde primaient les mythes et les croyances, tandis que la science n'y avait aucune place. Mais ce monde est révolu, car l'esprit de l'homme théorique l'a définitivement désenchanté. Parce que l'art ne peut plus provoquer autre chose que la nostalgie de temps révolus qui remontent à l'enfance de l'humanité, il ne reste dès lors qu'à en constater les limites et à admettre qu'il est devenu un remède inapproprié contre la culture moderne exaltant l'homme théorique. L'idée de Nietzsche est que, au lieu d'opposer au moderne des figures spectrales qui jamais n'auront la force de revivre car les conditions mêmes de leur existence ne sont plus, il vaut mieux combattre le moderne sur son propre terrain. Ce qui, plus concrètement, veut dire que le 
remède contre la culture moderne, n'est pas à chercher ailleurs que dans l'esprit moderne lui-même, c'est-à-dire dans la rationalité. Telle va donc être la thèse de Nietzsche au même moment où sa rupture avec le wagnérisme sera complètement consommée.

Il n'est pas inutile de rappeler que le thème de la « mort de l'art» que Nietzsche semble ici reprendre à son compte est en fait un thème hégélien et qui constitue même une charnière essentielle de la philosophie hégélienne de l'histoire. Or cela veut-il dire que ce thème ait le même sens chez Nietzsche et chez Hegel?

Nous le savons, Hegel considère la science, du moins telle que l'incarne sa propre philosophie rationnelle, comme la phase la plus achevée de l'histoire humaine. Ce qui, toujours selon Hegel, a nécessité le dépassement successif des formes plus anciennes de l'esprit, à commencer par celle de ses premiers âges, celui où l'art en était l'expression par excellence. Tels sont le sens et la nécessité de la «mort de l'art» dans la philosophie hégélienne de l'histoire. Mais telle n'est pas la philosophie de l'histoire de Nietzsche et c'est aussi pourquoi le thème <de $>$ la «mort de l'art » n'y a pas la même portée, ni le même sens, que chez Hegel.

Examinons brièvement ce point. La philosophie de l'histoire de Nietzsche lui a été fortement inspirée par Jacob Burckhardt qui occupait la chaire d'histoire et d'histoire de l'art à l'université de Bâle, à l'époque où Nietzsche y enseignait la philologie grecque. Burckhardt est essentiellement connu pour ses fameuses fresques des grandes civilisations dont celle consacrée à l'Histoire de la civilisation grecque et surtout celle consacrée à la Civilisation de la Renaissance en Italie (1860). L'approche de Burckhardt n'y a rien de comparable avec une théorie spéculative qui, à la façon de Hegel, chercherait à dégager une loi organique de l'histoire où chaque civilisation apparaîtrait comme une marche du progrès qui mènerait au présent ou à la modernité. Se refusant de considérer l'histoire par rapport au présent, Burckhardt tente au contraire de considérer chaque civilisation pour ellemême, dans l'unité de sa vie propre. Ce qui se dégage de telles considérations, c'est alors la prédominance d'un élément invariable : ce ne sont pas des principes rationnels de croissance - quelque chose comme une nécessité dialectique interne à la dynamique de l'histoire — qui détermine l'émergence d'une civilisation, mais c'est plus simplement l'inventivité d'un peuple dont les créations culturelles et institutionnelles - l'art, la religion, l'économie et la politique - ont convergé avec bonheur au lieu de s'affronter.

Cette conception de l'histoire sera systématisée par Burckhardt dans un cours donné à Bâle vers 1872 et qui sera édité à titre posthume sous le titre Considérations sur l'histoire universelle (1905). Nietzsche avait assisté à ce 
cours et en fut manifestement fort marqué. Au lieu de manifester une évolution de l'esprit qui avérerait quelque chose comme une raison agissant dans l'histoire, Burckhardt soutient que l'histoire à travers les grandes civilisations qu'elle a connues, manifeste au contraire un tout autre principe organisateur fondé sur la constance. Partout dans l'histoire, se retrouve un même principe invariable qui, de civilisation en civilisation, ne fait que se répéter. Cet invariant est simplement la nature humaine, avec ses passions, ses ambitions et ses œuvres. Mais si l'histoire est ainsi accordée au diapason de la nature humaine, celle-ci est elle-même accordée au diapason de la nature tout court. Ainsi, au lieu de faire la preuve d'un arrachement progressif de la raison à la nature, l'histoire fait la preuve inverse : toute entreprise humaine bat au même rythme que celui de la nature. C'est la dynamique naturelle qui scande la vie historique des hommes. Cette dynamique n'est pas linéaire mais cyclique, car, comme tous les cycles naturels, elle est faite d'une éclosion, d'une apogée et d'un déclin. C'est ce mouvement, on ne peut plus propre à tout ce qui est vivant, qui sans cesse se répète lui-même, de telle façon que l'histoire continue comme continue la vie ne général.

Dans la mesure où Nietzsche s'est inspiré de la conception de l'histoire de Burckhardt, on devine sans peine que, pour lui, les concepts de « mort de l'art», tout comme celui de "l'âge de la science», ne pouvaient avoir la signification que leur prête Hegel. En d'autres mots, il n'est pas question pour Nietzsche de considérer la science comme un progrès de l'esprit par rapport à l'âge où c'était plutôt l'art qui lui servait d'expression. Mais il est vrai par ailleurs que la science moderne ouvre, aux yeux de Nietzsche, une voie d'inspiration pour entreprendre la critique d'une certaine conception de la science, comme aussi la critique d'une certaine conception de l'art.

Cette voie d'inspiration, Nietzsche dit la trouver dans la méthode analytique qui prévaut dans les sciences de la nature et dans la chimie en particulier. Dès le premier aphorisme d'Humain trop humain, il écrit :

Tout ce dont nous avons besoin, et que nous ne saurions tenir que du niveau actuel de nos sciences, c'est une chimie des représentations et sentiments moraux, religieux, esthétiques, ainsi que de toutes ces émotions que nous ressentons en relation avec les grands et des petits courants de notre civilisation et de notre société (OC III/1, p. 23).

Or dans le même premier aphorisme, et dans plusieurs autres ensuite, Nietzsche met lui-même cette chimie en relation avec la méthode à adopter en histoire qu'il comprend dès lors comme une généalogie de la pensée et des œuvres. Que faut-il donc entendre par ce que Nietzsche nomme encore 
«l'histoire de la genèse de la pensée » (OC III/1, p. 35) ? Manifestement, parce qu'elle s'apparente à la méthode de décantation utilisée en chimie, la genèse dont il est ici question n'a pas pour but d'exposer les origines de l'histoire dans quelque chose qui serait de l'ordre d'un " grand commencement », c'est-à-dire une espèce de donnée de départ absolue qui aurait décidé de tout le destin de l'histoire humaine et qui resterait comme la mesure de son devenir. Une histoire dont l'origine serait de ce genre ressemble précisément à la conception hégélienne de histoire où la genèse s'apparente au triomphe initial de la raison sur la déraison, de la logique sur l'illogique, du bien sur le mal, de la vérité sur les apparences, de la liberté sur la nécessité, etc. À partir de cette origine, tout ce qui suit devient alors lisible comme une implémentation ou une mise en œuvre historiques de la raison, de la logique, du bien, de la vérité et de la liberté s'arrachant toujours un peu plus à leur contraire, et scandant de la sorte le progrès de l'histoire.

Mais, pour Nietzsche, la genèse historique ainsi conçue n'est qu'une chimère métaphysique. La vraie généalogie de l'histoire, la wirkliche Historie, ne consiste pas en une construction métaphysique de l'origine par pure représentation d'un grand commencement dont procéderait tout le progrès. Beaucoup plus proche de l'idée de Burckhardt, la thèse de Nietzsche est que la vraie généalogie de l'histoire doit être connaissance des élévations et des effondrements, des essors et des déclins, dont est faite toute vie en général, y compris donc la vie historique des hommes. En ce qui concerne celle-ci, la généalogie montre que tout ce que la représentation métaphysique tient comme opposé est profondément lié et que ce sont ces liens qui tissent la toile identique d'une histoire qui se répète différemment en mêlant toujours raison et déraison, logique et illogique, bien et mal, vrai et faux, liberté et nécessité. Voilà, explique Nietzsche, les « petites vérités discrètes » $(O C$ III/1, p. 25) que le généalogiste doit mettre au jour en adoptant pour cela la méthode du chimiste qui décante, analyse et déconstruit.

On peut donc aisément s'en rendre compte, l'esprit de la science, auquel Nietzsche se rattache désormais, est aux antipodes de ce $<$ que $>$ Hegel entendait par là. Au lieu d'être une œuvre de synthèse dans l'apothéose du savoir absolu, la science est pour Nietzsche une œuvre de décomposition et d'analyse des représentations humaines dans ce qu'elles prétendent avoir de plus grandiose, comme la religion, l'art, la moralité et la rationalité. Suivant la méthode du chimiste qui parvient à montrer que bien souvent les choses aux couleurs les plus magnifiques ne résultent que du mélange des matières les plus ternes, la déconstruction montre que, derrière ces moments de splendeur spirituelle que sont l'art, la religion, la morale et la philosophie, il n'y a que 
des choses « très humaines, trop humaines ». S'éclaire ainsi le titre de l'ouvrage de 1878. Sa thèse générale est donc simplement la suivante : les entités transcendantes - le vrai, le beau, le bon — dont la métaphysique prétend nous parler, ne sont au fait que des illusions très humaines qui, beaucoup plus modestement, procèdent d'un besoin tout aussi humain de transcender la vie, de s'illusionner à propos de tout ce que, en réalité, elle ne contient pas. Art, religion, morale et philosophie ne sont donc que des fictions qui procèdent d'un besoin d'enchanter la vie et, partant, de la faire passer pour ce qu'elle n'est pas. La métaphysique est un mensonge, mais, au fond, ce mensonge répond à un besoin vital de sublimation. La métaphysique est un mensonge vital.

Revenons donc au thème de «la mort de l'art» chez Nietzsche. De nouveau, il s'oppose assez manifestement à ce qui ressemble à la même idée chez Hegel. Pour celui-ci, l'art avait dans le passé une fonction métaphysique qui a été reprise, mais en même temps dépassée de façon plus rationnelle, par la science au sens philosophique et hégélien du terme. Pour Nietzsche, l'art est mort, car tout ce qui relève d'une ambition métaphysique est devenu désormais impossible. La «métaphysique d'artiste » n'était donc qu'un rêve dont les enchantements ne résistent plus à la déconstruction qui fait apparaître que l'art est lui aussi, comme toute expression métaphysique, un travestissement de la réalité qui répond au besoin très naturel de l'homme d'enchanter le monde pour sublimer la condition de son existence.

Avec Humain trop humain et les écrits qui vont suivre, Nietzsche entame sa destruction de la métaphysique et de toutes les valeurs qu'elle promeut. L'art ne fait pas exception. Comme la religion, la morale ou la philosophie idéaliste elle-même, l'art prétend nous porter jusqu'à l'en-soi du monde et nous en révéler l'essence profonde. Mais il ne s'agit que d'un leurre. Tout cela n'appartient qu'à la représentation et aucune représentation ne saurait nous porter plus loin qu'elle-même. Dans ces conditions, comment pourrions-nous prendre au sérieux la fonction que Schopenhauer attribuait à l'art? Pour que l'art puisse servir à nous faire voir un en-soi du monde, encore faudrait-il supposer qu'il y en ait un par-delà les apparences de notre monde visible. Mais ce postulat est faux ou, plus exactement, il relève des mirages et des fictions que produisent nos représentations.

Par rapport à métaphysique de Schopenhauer auquel il adhérait antérieurement, Nietzsche soutient désormais l'inverse : la vérité, si elle existe, se tient du côté de la représentation et certainement pas du côté du monde en-soi. Celui-ci n'est qu'une projection de la représentation et la prétendue vérité de cet en-soi plonge ces racines dans les illusions de nos représentations. C'est du 
côté des illusions engendrées par la représentation que semble donc se tenir la seule vérité. Comme le dit Nietzsche à l'aphorisme 29 d'Humain trop humain :

Qui nous dévoilerait l'essence du monde, nous infligerait à tous la plus pénible désillusion. Ce n'est pas le monde comme chose en soi, mais le monde comme représentation (comme erreur) qui est si riche de sens, profond, prodigieux, si gros d'heur et de malheur (OC III/1, p. 45).

Plutôt que de sonder les chimères métaphysiques, il s'agit donc pour Nietzsche de comprendre comment les hommes en viennent à imaginer de telles chimères. En d'autres mots, quels besoins les poussent à projeter des mondes transcendants par-delà le monde des phénomènes qui leur est pourtant le plus proche. C'est ce sur quoi doit justement servir à faire l'éclairage une psychologie fondée sur la méthode généalogique d'analyse et de décomposition telle qu'on l'utilise dans certaines sciences positives comme la chimie. Cette psychologie montre que toutes les fictions métaphysiques répondent à un besoin instinctuel de l'homme. Elles lui sont tout simplement nécessaires et indispensables pour vivre, comme si, pour vivre, l'homme avait besoin de croire en toutes ses valeurs qu'il projette dans un au-delà de sa propre vie en évoquant un bien en soi, un beau en soi, un vrai en soi. Il y a donc, comme le dit Nietzsche, une nécessité tellement vitale de l'illusion que nous perdons le plus souvent de vue que ce ne sont que des illusions. Au fond, le souci de vérité n'est pas inscrit au fond de la nature humaine, car ce souci déforce le besoin de fantasmer d'autres mondes qui eux-mêmes ne sont que les réponses au besoin de trouver du plaisir malgré le déplaisir de l'existence. Pour Nietzsche, l'art fait désormais partie de la panoplie dont se sert notre imaginaire pour répondre à ce besoin, et c'est pourquoi l'art ne pourrait échapper lui non plus à l'examen généalogique. Apparaît alors que, lui aussi, $<$ il $>$ ne concourt qu'à nous présenter la réalité autrement qu'elle n'est, en l'embellissant et en nous rendant les choses beaucoup plus attrayantes qu'elles ne sont. Qui plus est, la puissance illusionniste de l'art est de parvenir à travestir notre propre vie en nous la faisant voir, elle aussi, comme une œuvre d'art.

L'art rend supportable le spectacle de la vie en la recouvrant d'un voile de pensée trouble (OC III/1, p. 122).

La déconstruction des chimères métaphysiques dans Humain trop humain vise bien à un désenchantement du monde. Cette déconstruction a la forme d'une sorte de dévoilement du monde de façon à disperser les brumes et les nuages dont l'esprit, sur son mode idéaliste, l'a délibérément auréolé. Mais, pour Nietzsche, cette déconstruction est aussi la promesse d'une aurore nouvelle de 
la pensée que, au dernier aphorisme de Humain trop Humain, il nomme la «philosophie d'avant-midi» (OC III/1, p. 306). C'est son idée qui sera au centre des deux ouvrages que Nietzsche publiera par la suite: Aurore (Morgenröte) en 1881 et Le Gai savoir (Die fröhliche Wissenschaft) en 1882.

Le désenchantement du monde, qui équivaut à l'affranchissement par rapport à toute métaphysique, dit donc aussi l'avènement du «libre esprit ». Cet esprit, pour Nietzsche, est celui qui a enfin appris à reconnaître que le surhumain qu'il vénérait n'est qu'une production de l'humain. La déconstruction n'a dès lors pour seul effet que de ramener l'homme à lui-même et de lui apprendre à vivre dans la proximité de soi. Cette nouvelle conscience de soi est conscience que le surhumain est profondément humain. Peut-être que ce savoir n'a pas encore l'ambition d'aller plus loin, mais en abrogeant toute solennité, il rend l'esprit léger en le libérant du poids de la transcendance. La Gaya scienza ne veut exprimer que cela : la science rend le cœur de l'homme léger car, en balayant son ciel des nuées idéalistes, elle dissipe toutes les illusions des puissances surhumaines et laisse l'homme à lui-même sous un ciel serein. De ce ciel, il sait désormais qu'il n'a rien à attendre. Toutefois, ce désenchantement ne conduit pas au scepticisme. L'abrogation des anciennes vérités, le dévoilement de la transcendance comme simple apparence, bref la sécularisation du monde, est au contraire porteuse d'un nouvel enthousiasme face à la nouvelle image que l'homme découvre ainsi de lui-même. Cette image n'est plus celle de l'homme se prosternant devant les puissances surhumaines, ce n'est plus l'ancienne image de l'homme qui se soumettait à la loi morale et cherchait à atteindre par l'art ou la philosophie ce qu'il y aurait derrière les apparences. La nouvelle image est celle de l'homme qui comprend que le surhumain n'est que la dimension cachée de lui-même, et cette image nouvelle le conforte à se sentir lui-même comme un homme absolument libre. Le saint, l'artiste et le sage étaient les images produites naguère par l'homme pour se représenter sa propre grandeur, mais cet homme était totalement aliéné par ces propres productions dont précisément il oubliait être l'auteur. L'esprit libre, au contraire, ramène le saint, l'artiste et le sage à la conscience de soi, et, par celle-ci, l'homme se débarrasse lui-même de l'image qu'il avait de sa grandeur dans l'image du saint, de l'artiste et du sage. Il peut se voir désormais tel qu'il est et découvre qu'il est le créateur des valeurs, tout en sachant qu'il peut aussi en créer de nouvelles.

La fin de l'idéalisme corrobore l'avènement de la Gaya scienza et celleci n'est que la conscience de soi de la liberté de l'homme. Mais, somme toute, cette liberté n'est encore comprise par Nietzsche que comme une autoaffirmation de l'homme en tant que créateur. Rien n'est dit, par contre, concernant la création de l'homme lui-même, sur ce qu'il cherche et ce qu'il 
veut en créant. L'esprit libre ne s'est affirmé jusqu'ici qu'en se libérant de l'idéalisme et des valeurs métaphysiques. Il s'est affirmé par la négation de ce qui l'aliénait, mais, par là, nous ne trouvons pas encore l'affirmation de ce qu'il veut. Après la «philosophie d'avant-midi », l'explicitation de la volonté créatrice de l'homme sera le principal objet de la «philosophie de midi» de Nietzsche. Elle se trouve exposée dans Also sprach Zarathustra dont les deux premières parties paraissent un an après Le Gai savoir en 1883, et les deux parties suivantes en 1884 et 1885 . On peut considérer cet ouvrage comme la plus grande œuvre de Nietzsche, celle où se trouve sa philosophie définitive. Les écrits ultérieurs ne seront qu'autant de développements des thèses essentielles exprimées, plutôt qu'exposées, dans le Zarathoustra.

\section{La philosophie du Zarathoustra}

L'ouvrage se présente sous la forme d'un long poème en prose relatant les prophéties de Zarathoustra qui, en langue perse ancienne, est le nom de Zoroastre, fondateur de la religion qui porte son nom. Pourquoi avoir choisi ce personnage ? Nietzsche y répondra lui-même, dans son ouvrage autobiographique de 1888 Ecce Homo, en disant que, puisque Zarathoustra passe pour être le premier à avoir enseigné la morale en distinguant le bien du mal, il fallait aussi lui rendre symboliquement la parole, pour qu'il abolisse sa propre doctrine et aboutisse à son contraire, c'est-à-dire, dit Nietzsche, « à moi ».

La première partie de Zarathoustra commence en quelque sorte par faire état de la situation à laquelle a conduit la philosophie d'avant-midi. Le premier discours de Zarathoustra est en effet intitulé « Des trois métamorphoses »:

De l'esprit, c'est trois métamorphoses que je vous nomme : comment l'esprit devient chameau, et lion le chameau et, pour finir, enfant le lion (OC VI, p. 37).

L'allégorie n'est pas bien difficile à comprendre. Le chameau, c'est l'esprit de vénération; il correspond à l'homme qui porte sur son dos le fardeau des transcendances et qui a appris à se prosterner devant elles. Bref c'est l'homme de l'idéalisme, celui qui ne veut pas se contenter de la vie quotidienne qu'il juge sans pesanteur, mais qui veut au contraire assumer des tâches dans lesquelles il croit se grandir lui-même : obéir à des dieux, se soumettre aux valeurs éthiques, esthétiques et philosophiques. Le chameau est l'esprit qui a fait du saint, de l'artiste et du sage, ses modèles d'existence. Mais sitôt a-t-il pris le chemin du désert, le chameau s'aperçoit que sa marche serait autrement plus légère et plus facile sans ces fardeaux qui pèsent sur son dos et dont il est 
devenu l'esclave. Prenant conscience ainsi de son aliénation, il décide de s'en débarrasser. C'est le moment où il se transforme en lion et prend conscience de sa liberté.

Cette liberté, le lion l'affirme en opposition à tout ce qui l'aliénait avant sa métamorphose. Il clame à présent un «je veux» et, en un rugissement, l'oppose au « tu dois » qui était la règle du chameau. Mais ce « je veux » ne semble exprimer encore que l'obstination de l'auto-affirmation : la volonté ne veut ici qu'elle-même en niant toute forme d'aliénation. En d'autres mots, ce qui manque encore au lion, c'est l'expression positive de ce qu'il veut. Prenant dès lors conscience des limites de sa propre volonté, le lion comprend désormais que l'objet de sa volonté est essentiellement la création. Et, pensant de la sorte, il se métamorphose en enfant. Pourquoi en enfant? Parce que, dit Nietzsche, la création est un jeu qui ne demande d'autre disposition d'esprit que l'innocence et l'oubli, et cet état d'esprit est bien celui de l'enfant qui crée en jouant et qui joue en créant. Il crée son propre monde et cette création est ludique car elle est totalement libre, autant qu'elle est innocente car sans arrière-pensées.

La première métamorphose, qui du chameau a fait passer au lion, coïncide avec ce que Nietzsche appelle « la mort de Dieu », c'est-à-dire la fin de toutes les idéalités qui auraient la forme d'un au-delà de l'homme, d'une transcendance qui dominerait et illuminerait l'existence humaine. Mais, avec la mort de Dieu, la tendance idéaliste ne disparaît pas nécessairement. Il se pourrait qu'elle subsiste sous la forme de la conscience d'être l'auteur des idéaux qui étaient naguère confinés dans un au-delà de l'homme. C'est l'attitude que Nietzsche appelle celle du « dernier homme » (OC VI, p. 27) qui se sait créer les valeurs auxquelles il continue de se soumettre. À l'ignominie du « dernier homme », Nietzsche oppose l'attitude du « surhomme». Celui-ci sait la mort de dieu mais il a refusé aussi de vouloir lui substituer les nouveaux dieux nés de son esprit. Le surhomme sait que toute idéalité n'est que la chimère d'un ciel utopique et c'est vers la terre qu'il se tourne désormais. Sa liberté, il veut l'enraciner dans la terre et, comme tout ce qui naît de la terre, il veut une liberté fidèle à la terre.

Que signifie cette injonction de « la fidélité à la terre » (OC VI, p. 24) introduite dès le prologue du Zarathoustra ? La terre ici en question signifie assez clairement le sein d'où naît toute vie qui s'étend dans l'espace et le temps. La terre n'est donc pas à comprendre comme une masse matérielle ou comme la somme de tout ce qui est. Elle est le terreau d'où surgit et où se développe tout ce qui vit. La terre est en ce sens puissance créatrice, elle est poièsis. Son seul et unique principe est de laisser être, de favoriser la vie, et tout ce qu'elle engendre participe de ce principe. Tout est vouloir vivre, 
aspiration à croître, à s'épanouir, à s'enrichir, à devenir toujours plus fort et plus puissant. C'est le même élan vital, la même pulsion de vie, qui, malgré les dangers, les périls, les limites, la fragilité, la corruptibilité et la finitude, caractérise tout ce que la terre laisse surgir. Aussi, le «surhomme» qui n'advient qu'après « la mort de Dieu » est celui qui prend conscience de la nature de sa propre liberté et saisit qu'elle ne diffère pas de cet élan qui caractérise tout ce que porte la terre. En d'autres mots, le «surhomme» comprend que sa liberté est inséparable du vouloir vivre ou, selon les propres termes de Nietzsche, inséparable de la "volonté de puissance ». Si donc la «mort de dieu » était le thème principal de la première partie du Zarathoustra, il n'est pas étonnant que « la volonté de puissance » soit le thème principal de sa deuxième partie. Bien sûr, la volonté de puissance qui importe ici est plus précisément celle qui concerne cet homme, le "surhomme », qui a retrouvé l'esprit d'un enfant et qui, comme l'enfant, est essentiellement créateur. À l'égard des choses et du monde en général, cet homme a une attitude « originaire » ou " primaire», car il crée de nouvelles mesures et donne une forme nouvelle à tout ce qui l'entoure. Pour cet homme, le monde n'est pas un donné, mais il est à faire. C'est aussi pourquoi, avec lui, le monde est toujours à venir. Comme le dira Nietzsche dans Le Crépuscule des idoles, un texte plus tardif publié en 1889: "Le monde vrai est une fable». Cette phrase est clairement ambiguë. D'un côté, elle entend rappeler que la vérité du monde dont la métaphysique veut nous parler n'est qu'une fable. Mais, d'un autre côté, que le monde vrai soit devenu une fable, signifie également qu'il n'est rien d'autre que le monde produit par les fictions de l'activité humaine. En d'autres termes, la vérité du monde coïncide seulement avec les apparences que le « surhomme » invente en créant le monde du même coup.

Par sa propre création ou son activité de fiction, le « surhomme » se sait intimement lié à la force créatrice de la terre et c'est aussi la raison pour laquelle cet homme doit prendre le temps au sérieux. Comment comprendre cela ? La métaphysique a toujours exclu le temps de l'ordre ontologique, considérant les caractéristiques du temps comme opposées aux caractéristiques de l'être, de la même façon que ce qui est éphémère et donc voué au néant est opposé à ce qui est pérenne, immuable, et qui possède le caractère absolu des idéalités. Au contraire, l'homme créateur ou le « surhomme » sait désormais qu'il doit toujours compter avec le temps. En effet, sa volonté de puissance n'est soumise à rien, si ce n'est justement au temps et à son propre rythme qui partout, de l'aurore au déclin, impose le changement continu. Aussi le surhomme qui se sait appartenir à la terre se sait aussi être dans le temps. Il sait que, comme tout ce qui procède de la vie, il est profondément temporel. Par là, il est conscient du fait que sa propre vie créatrice est marquée par la 
finitude, que ses créations seront toujours éphémères, que ce qu'il construit sera détruit et qu'il lui faudra donc reconstruire. Il sait ainsi qu'il aura toujours à se dépasser sans cesse, à briser ce qu'il a été en cherchant à être ce qu'il n'est pas encore. Bref, il sait que sa volonté de puissance fait de sa propre existence un projet continu et incessant car inabouti, donc un projet qui ne pourra jamais se satisfaire d'aucune réalisation, car toujours il voudra dépasser ce qu'il a atteint.

La vraie question devient dès lors la suivante : ce dépassement incessant, qui fait de la volonté de puissance un mouvement ininterrompu sans aucune perspective d'un repos possible, ne finit-il pas par avoir un effet paralysant sur la volonté de puissance elle-même ? L'idée que tout projet est voué à une reprise sans fin n'engendre-t-il pas un sentiment de découragement et, avec celui-ci, l'extinction progressive de l'ardeur créatrice face à ce qui ressemble au non-sens d'une vie enchaînée à la course du temps ? Faut-il alors conclure que la volonté de puissance est simplement vouée à s'éteindre ellemême dans un sentiment d'impuissance, et que, avec ce sentiment, la vie devient un fardeau si lourd que la souffrance finit par l'emporter sur le plaisir de la création? Comme nous l'avons vu, c'est justement ce qu'a pensé Schopenhauer à propos du monde comme volonté. Mais telle n'est certainement pas la position de Nietzsche dans le Zarathoustra.

Le dernier chapitre de la deuxième partie de l'ouvrage a pour titre «L'heure la plus silencieuse » et c'est précisément le moment où le temps luimême apparaît en sa propre essence. Comme nous l'avons vu, le temps ressemble à une suite ininterrompue de moments immédiatement absorbés par le passé tandis que le futur recule sans cesse devant la volonté de puissance qui se dépasse elle-même en de nouveaux projets sans fin. Mais ne serait-il pas possible de parvenir à une autre compréhension du temps qui permettrait aussi de penser de façon plus profonde sa relation intime à la volonté de puissance ? Le moment de cette révélation doit être sans doute «l'heure la plus silencieuse », car elle exige tout simplement de mettre en sourdine l'agitation et l'effervescence de la vie, d'en réduire le bruit, afin de laisser entendre le cours silencieux du temps en sa véritable énigme. C'est cette idée qui va faire le thème central de la troisième partie du Zarathoustra.

Cette troisième partie est précisément consacrée à la doctrine de « l'éternel retour » qui, au dire de Nietzsche dans Ecce Homo, doit être considérée comme le noyau de toute l'œuvre. L'idée de l'éternel retour peut sans doute être résumée par ces lignes :

Toujours menteuse est la ligne droite... Courbe est toute vérité, le temps luimême est un cercle (OC VI, p. 177). 
Mais surgit aussitôt la question de savoir pourquoi l'infinité du temps devrait être conçue plutôt comme un cercle que comme une ligne continue et irréversible orientée vers le futur? Pour le comprendre, il suffit de considérer plus avant ce qu'est le futur. On admettra de le caractériser comme ce qui arrive et qui signe son advenue par son entrée dans le présent et, tout aussitôt, son enfouissement dans le passé. En ce sens, le futur est donc ce qui continuellement génère du passé, tandis que, inversement, le passé ne résulte de rien d'autre que de la disparition et de l'engloutissement du futur. Or la conception linéaire du temps ne permet donc pas de voir ainsi que le passé est la substance du futur et le que le futur est la substance du passé. Le temps ressemble donc beaucoup plus à un cercle où s'abolit toute division du passé et du futur, puisque le passé a les traits de l'avenir et l'avenir les traits du passé. En d'autres mots, tout ce qui a été devra revenir et, de la même façon, tout ce qui doit arriver ressemble déjà à ce qui a été. C'est simplement cette répétition du temps que cherche à exprimer l'idée de l'« éternel retour ».

Pourrait-on dire, cependant, que cette conception du temps est plus encourageante que la conception linéaire selon laquelle la volonté de puissance s'épuise dans ses incessants projets? Il se pourrait, en effet, que l'idée de l'« éternel retour» reste tout aussi oppressante, puisque, si tout est appelé à revenir et à recommencer, le sort de l'activité créatrice du surhomme devient comparable au sort de Sisyphe. Dans le Zarathoustra, l'image utilisée pour exprimer l' «éternel retour » est celle du serpent qui se mord la queue et la même image du serpent s'introduisant dans la gorge du « surhomme » sert à exprimer le sentiment d'oppression et d'étouffement qui s'empare de l'homme créatif. Mais, d'après Zarathoustra, il était pour ainsi dire nécessaire que le serpent s'introduise dans la bouche de cet homme créatif pour que celui-ci puisse ainsi lui mordre la tête. Cette allégorie du Zarathoustra veut simplement exprimer la rédemption de l'idée de l' « éternel retour » qui, d'abord, paraissait oppressante et qui, à présent, est devenue bien plus légère en procurant un sentiment d'exubérance débordante. Que veut dire, en effet, mordre la tête du serpent et pourquoi cela procure-t-il un tel sentiment de légèreté ? Mordre la tête du serpent signifie clairement tirer le meilleur parti de la vie ou, comme le dit encore l'expression française, mordre la vie à pleines dents. Et c'est justement cela qui s'accompagne du dépassement de l'angoisse d'abord ressentie à l'égard de l'«éternel retour ». Ce dépassement provient simplement de la reconnaissance de la structure dionysiaque du temps ou de la vie qui est de faire croître, mais aussi de détruire chaque chose en un jeu incessant. Dès lors, pour l'homme créatif, affirmer sa propre volonté face à la répétition du temps est la seule façon de dire « oui » à la vie et d'accepter son continuel 
va-et-vient entre floraison, fanaison et finalement disparition. Cette acceptation ne réalise rien d'autre que la conciliation de la liberté et de la nécessité, l'union de la création et de la répétition, comme seule règle de la vie. La volonté de puissance de l'homme ne peut que vouloir le retour éternel du même, car c'est au fond de ce dernier que la puissance créatrice de l'homme tire sa propre puissance. De savoir l'éternel retour, et de l'assumer pleinement, l'existence s'engage totalement dans le grand jeu du monde. La liberté de sa création choisit de s'unir au jeu du temps lui-même.

Chez Schopenhauer, douleur, souffrance et donc pessimisme étaient la conséquence de ne voir que la répétition du temps sans aucune rédemption possible. À cela, Nietzsche oppose la joie et l'optimisme qui proviennent d'une vision plus profonde : en reconnaissant l'éternel retour, cette vision reconnaît aussi une éternité dans l'essence du temps. Comme Chronos dans l'ancienne mythologie grecque, le temps dévore ses enfants, il anéantit tout et rien ne lui résiste. Mais la joie résulte d'une vue moins courte qui sait reconnaître que la destruction n'est que la contrepartie du jeu inépuisable du temps ou de la vie. La joie est justement ressentie lorsque nous parvenons à voir de l'éternité, certainement pas au sens transcendant ou métaphysique, mais une éternité qui est celle du monde lui-même.

Aussi, la quatrième et dernière partie du Zarathoustra nous le montre animé de cette joie face à ceux qui, désorientés par la mort de Dieu, en sont restés au sentiment de vide et de rien. À ces hommes, l'unique et dernière leçon de Zarathoustra est d'abandonner toute nostalgie pour le rire, car le rire est l'extériorisation de la joie et il ne peut y avoir que joie face à la découverte que l'éternité est inscrite en toute chose, car toute chose est liée à la vie et à son éternel renouveau. La joie vient de vouloir ainsi que tout recommence et cette joie n'est pas diminuée par la douleur et la mort, car la douleur et la mort font aussi partie de tout ce qui est lié à la vie.

\section{Le nihilisme accompli}

Le dépassement de la métaphysique est sans aucun doute un thème axial de toute la pensée de Nietzsche. Il est omniprésent depuis la critique du nihilisme socratique et platonicien dans La Naissance de la tragédie, jusqu'aux thèmes de «l'éternel retour» et de la "volonté de puissance » dans le Zarathoustra. Soutenir en effet que «l'essence du monde est volonté de puissance» (Pardelà bien et mal, 1886, § 186) ou bien encore que « l'essence la plus intime de l'être est volonté de puissance » (La Généalogie de la morale, 1887, § 12), c'est soutenir une thèse ontologique qui veut surmonter le déni de la vie dans 
lequel Nietzsche voit la marque la plus caractéristique de la métaphysique traditionnelle. La volonté de puissance est sans aucun doute un autre nom pour Dionysos, et c'est bien le principe dionysiaque de la vie que Nietzsche veut restaurer contre l'apollinisme philosophique, c'est-à-dire contre le règne illusoire des représentations conceptuelles. De même, soutenir la thèse de l'éternel retour, c'est redonner au devenir la signification qu'il perd lorsqu'il est seulement considéré comme procès de néantisation du temps. De nouveau, cette conception est celle de la métaphysique traditionnelle qui, au temps et au devenir, oppose l'absoluité de la présence, celle de l'idea chez Platon, celle de l'ousia chez Aristote, celle assumée par le cogito chez Descartes ou par la raison chez Hegel, etc. La thèse de " l'éternel retour » est donc, elle aussi, radicalement anti-métaphysique, car, à travers elle, Nietzsche ne cherche qu'à défendre une conception du temps qui porte la marque même de l'être ou de la vie: « Dire que tout revient, c'est rapprocher un monde du devenir de celui de l'être » (La Volonté de puissance, § 617).

Mais peut-on dire que, malgré ses intentions, Nietzsche parvient à sortir de la métaphysique pour autant? Parmi les plus grands commentateurs de Nietzsche figure très certainement Heidegger qui, dans le copieux ouvrage en deux volumes qu'il lui consacrera en 1961, montrera que ce n'est pas le cas. Pour Heidegger, l'œuvre de Nietzsche marque bien la fin de la métaphysique, mais elle n'est pas encore son dépassement, ni donc la sortie hors de la métaphysique. C'est pourquoi, selon la lecture de Heidegger, il s'agit plutôt de comprendre comment la métaphysique traditionnelle arrive à sa conclusion chez Nietzsche et s'y manifeste aussi dans son essence nihiliste la plus profonde.

Le nihilisme de la tradition philosophique occidentale est compris par Heidegger comme l'oubli de l'être (Seinsvergessenheit). À propos de cette notion, Heidegger s'explique assez clairement dans un texte repris dans les Holzwege de 1950 et qui a précisément pour titre « Le mot de Nietzsche "Dieu est mort" ». Voici ce qu'il dit :

Oublieuse de l'être et de sa propre vérité, la pensée occidentale pense, dès son début, constamment l'étant en tant que tel. Depuis, elle n'a pensé l'être qu'en pareille vérité, de sorte qu'elle a porté ce nom à la parole que fort maladroitement... Cette pensée oublieuse de l'être même, tel est l'événement simple et fondamental, et pour cela énigmatique et inéprouvé de l'Histoire occidentale, qui entre-temps est sur le point de s'élargir en Histoire mondiale. Au bout du compte, l'être est tombé, dans la Métaphysique, au rang d'une valeur. Ceci nous est témoignage de ce que l'être en tant qu'être n'est pas reçu (Chemins qui ne mènent nulle part, <trad. fr. W. Brokmeier, Paris, Gallimard, 1962,> p. 212213). 
Ce texte pourrait être mis en rapport avec ce que Heidegger dit de la pensée présocratique, et plus particulièrement celle de Parménide. Dans un texte de 1954 intitulé Was heisst Denken ?, Heidegger soutient que la thèse originaire de la pensée grecque était, selon le mot du sixième fragment du poème de Parménide, de « dire et penser l'être à propos de ce qui est ». Ce qui voulait seulement dire que ce qui requiert avant tout la pensée, c'est l'être. Tel est bien ce que, d'après Heidegger, devait signifier le fameux fragment VIII qui dit : to gar auto estin noein te kai einai. Il indique que, originairement, était reconnue et admise la co-appartenance ou l'étroite proximité de la pensée (noein) et de l'être (einai).

Toutefois, cette première approche concernant la tâche de la pensée est perdue dans ce que recouvre le titre de métaphysique, lorsqu'il est, pour la première fois, défini par Aristote comme question portant sur ce qui est en général (ti to on). Dans le poème de Parménide, la question était originairement dirigée vers la vérité de l'être et, depuis les débuts de la métaphysique, elle est détournée en direction de la vérité de l'étant (to on). Autrement dit, l'essence de la métaphysique repose dans une question directrice qui est celle de la vérité de l'étant et donc dans l'abandon ou l'oubli d'une question qui, elle, ne s'y trouve jamais posée : la question de l'être. Comme l'exprime déjà Heidegger dès les années vingt, donc à l'époque de Sein und Zeit, la métaphysique traditionnelle a de la sorte toujours été oublieuse de la "différence ontologique », car jamais elle n'a pris en compte la différence qui fait que l'être n'est pas un étant. L'étant nous est bien connu, c'est l'ensemble des choses autour de nous. Mais l'être dont nous utilisons le nom pour dire les choses en général et qui, lui, ne se trouve manifestement pas parmi les choses, est tout simplement laissé pour compte et négligé. Plus exactement, la métaphysique a seulement pensé l'être à partir de l'étant, et, partant, elle a dévoyé le sens de la question qu'elle aurait dû se poser en tant qu'ontologie. Plutôt que de porter sa question en direction de l'être comme tel, la métaphysique l'a portée en direction des choses en général pour se demander ce qu'était leur fondement, ce qu'était le sol ou le principe à partir duquel elles peuvent être. Comme l'exprimera encore Heidegger, la métaphysique a ainsi fait de l'étantité (Seiendheit) de l'étant sa question essentielle, sans considérer que la question de l'être n'était pas du même genre.

À plusieurs endroits de son Nietzsche de 1961, et notamment au chapitre $\mathrm{V}$ qui a précisément pour titre Le nihilisme européen, Heidegger fournit une longue fresque assez détaillée de cet oubli de la question de la vérité l'être et de sa confusion avec la question de la vérité ou du principe fondamental de l'étant. Il montre ainsi comment, dès Platon et Aristote, l'être a été compris en termes d'idea ou d'ousia pour dire le fondement permanent, la substance ou 
l'essence identique, qui assure aux choses leur possibilité d'être présentes et de se tenir sous notre regard. Avec la philosophie chrétienne au Moyen Âge, le fondement de l'étant sera attribué à un étant particulier, à savoir <à > l'étant suprême ou $<\mathrm{au}>$ Dieu créateur de la foi chrétienne, par rapport auquel tous les autres étants sont le produit et sont donc aussi redevables de leur propre existence ou de leur actualitas. À l'époque moderne, le fondement de tout ce qui est deviendra enfin le sujet au sens de l'homme pensant, le cogito ou la raison, dont la représentation assure la constitution de toutes les choses en tant que simples objets.

Or, selon Heidegger, pour quelle raison la philosophie de Nietzsche ferait-elle partie de cette tradition métaphysique de l'oubli de l'être et en quel sens en serait-elle même la conclusion ? Heidegger montre tout d'abord que Nietzsche ne se désolidarise pas de la conception traditionnelle de l'être comme principe fondamental et permanent de l'étant en général. Mais le point le plus important est que ce principe est désormais conçu par Nietzsche comme « volonté de puissance ». Dans les fragments posthumes de Nietzsche on peut lire ce texte qui résume bien sa position ontologique :

La vie qui est pour nous la forme la plus connue de l'être est spécifiquement une volonté d'accumuler de la force : tous les processus de la vie ont ici leur levier... La vie tend à la sensation d'un maximum de puissance; elle est essentiellement une tension, une aspiration vers plus de puissance ; la tension n'est rien d'autre que la tension vers la puissance ; cette volonté demeure ce qu'il y a de plus profond et de plus intime (La Volonté de puissance, frag. 689).

On peut donc dire que, avec la philosophie de Nietzsche, la dernière époque de la métaphysique est atteinte, lorsque l'être de l'étant en son ensemble équivaut au règne de la volonté de puissance ou encore au règne de la vie qui a pour seul principe la pure extension de sa propre puissance et pour loi de rendre toujours plus effective cette puissance. Comme le commente Heidegger :

La puissance ne s'exerce que dans la mesure où elle est maîtresse, où elle règne au-dessus du degré de puissance chaque fois atteint. La puissance n'est puissance et ne le reste qu'aussi longtemps que demeure accroissement de puissance et se commande le surplus (das Mehr) dans la puissance... Il appartient à l'essence de la puissance de se surpasser. Ce surpassement provient de la puissance même du fait qu'elle est commandement et qu'elle se donne elle-même le pouvoir, en tant que commandement, de surpasser le degré de puissance chaque fois atteint. Ainsi la puissance est-elle constamment en chemin vers elle-même, non seulement vers un degré de puissance supérieur, 
mais surtout vers la prise de pouvoir de sa pure essence (Nietzsche $<$, tome $>I I$, $<$ trad. fr. P. Klossowski, Paris, Gallimard, 1971,> p. 214).

Mais, dans ces conditions, qu'advient-il de ce qui est ou de l'étant en général, si le principe permanent qui le sous-tend est désormais la volonté de puissance et sa perpétuelle tension vers toujours plus de puissance, plutôt que ce que voulaient dire les notions traditionnelles d'idea, d'ousia ou de subjectum ? À cette question, voici la réponse de Nietzsche : l'étant en général n'est plus que le moyen dont se sert la volonté de puissance pour assurer sa perpétuelle ascension vers un surcroît de puissance. Dit autrement, l'étant en général ne se présente plus autrement que comme l'ensemble des valeurs que promeut la volonté de puissance. La volonté de puissance est institution des valeurs par lesquelles elle peut être puissance et ainsi se réaliser elle-même. Comme le dit Heidegger à propos de la thèse de Nietzsche :

L'institution des valeurs, c'est le processus fondamental de la vie, c'est la manière dont la vie accomplit et réalise son essence (Nietzsche $<$, tome $>I$, $<$ trad. fr. P. Klossowski, Paris, Gallimard, 1971,> p. 544).

Cela veut dire également qu'il n'existe pas de valeurs déjà établies ou de valeurs « en soi » qui précéderaient la volonté de puissance et sous l'autorité desquelles celle-ci aurait à se ranger. C'est précisément en ce sens que Nietzsche a entrepris sa critique des valeurs suprêmes que faisait valoir la métaphysique depuis Platon. De ce fait, la tradition lui apparaît comme cet état maladif où la vie, au lieu de jouir de l'absoluité de sa puissance, s'aliène et se nie elle-même face à des puissances supérieures illusoires. Pour Nietzsche, au contraire, les valeurs sont partout relatives à l'exercice de la volonté de puissance : elles sont les conditions de cet exercice. Mais, en même temps, ces conditions sont elles-mêmes conditionnées par l'exercice de la volonté de puissance, puisque les valeurs n'existent que par et pour cette puissance.

Dès lors, ce que Nietzsche appelle la «transvaluation de toutes les valeurs » ne peut être que la conscience de ce que sont les sources et les fonctions essentielles des valeurs que la vie, s'invente afin seulement d'affirmer sa propre puissance. Cette prise de conscience est reconnaissance que toutes les valeurs sont instituées par l'homme et qu'elles sont donc dépendantes de sa propre volonté de puissance. C'est de cette façon que l'homme devient le " surhomme » et vit enfin sa propre existence au rythme de l'être ou de la vie en général.

Or considérons à présent la conséquence la plus immédiate de cette transvaluation de toutes les valeurs. Manifestement, cette conséquence 
concerne l'accès que nous avons aux choses ou à ce qui est en général. Dans la tradition, cet accès était régi par l'idéal de vérité, et la vérité désignait l'objectivité de la connaissance en tant qu'elle atteint effectivement ce qui est. C'est cette conception de la vérité qu'abroge la volonté de puissance. $\mathrm{Ne}$ cherchant que sa propre satisfaction, la volonté de puissance ne se rapporte plus aux étants que dans ce but, et c'est toujours dans ce but qu'elle établit les conditions de sa propre représentation. Ce qui veut dire que tout acte de représentation, quel que soit son objet, est avant tout une appréciation ou une évaluation. Cette évaluation revient à accorder à l'étant la valeur qu'il a pour la volonté de puissance. Mais si tout ce qui est en général n'a de valeur et de sens que pour autant qu'il serve aux desseins de la volonté de puissance, si l'étant ne peut exister qu'à la condition que la volonté en tienne compte pour ses propres fins, cela signifie aussi que tenir quelque chose pour vrai, ou plus simplement encore le considérer comme étant, revient à le tenir pour appréciable ou évaluable.

Or, selon Nietzsche, tenir quelque chose pour vrai ou le considérer comme étant n'est qu'une falsification que nous faisons sous la pression des besoins vitaux de la volonté de puissance. Il s'ensuit que tout notre système cognitif n'est qu'un appareil de falsification. Déjà, la perception est inévitablement fausse et falsificatrice, car elle naît d'un besoin de figer les choses alors qu'elles sont en continuel mouvement. Mais, bien qu'elles soient fausses, nos représentations stabilisantes n'en sont pas moins nécessaires. Cette nécessité est à nouveau prescrite pas les besoins de la vie, puisque l'exercice de la volonté de puissance a souvent besoin d'instaurer autour d'elle l'illusion de la stabilité et de la permanence. Comme l'écrit Nietzsche dans ses Fragments posthumes :

La volonté de vérité est la volonté de rendre fixe, de rendre vrai, durable, d'escamoter ce caractère faux, de le transformer en lui donnant le sens de l'étant. La "vérité » n'est donc pas quelque chose qui serait déjà là et qu'il s'agirait de trouver, de découvrir, mais quelque chose qu'il faut produire et qui fournit un nom pour un processus indéfini ; mieux un nom pour une volonté qui veut indéfiniment régner en maître... La volonté de vérité, c'est bien plutôt l'un des noms pour la « volonté de puissance » (La Volonté de puissance, XVI, frag. 552).

Si la vérité n'est qu'un mensonge nécessaire, c'est seulement parce qu'elle appartient aux valeurs établies par la volonté de puissance pour son propre accroissement. Aussi la vérité ne figure-t-elle nulle part ailleurs que dans le registre des valeurs que Nietzsche appelle encore les « valeurs d'art », puisque, d'après lui, l'art est finalement $\langle\ldots>$ Nous l'avons vu, les dernières 
méditation $<\mathrm{S}>$ nietzschéenne $<\mathrm{s}>$ sur 1 'art $<\ldots>$ est la grande condition de possibilité de la vie... le grand stimulant de la vie » (La Volonté de puissance, frag. 853) ${ }^{1}$.

Par conséquent, lorsque Nietzsche dit que « nous avons l'art pour ne pas périr de la vérité » (La Volonté de puissance, frag. 822), il entend bien nous dire que l'art traduit au mieux le jeu fictionnel de la volonté et nous sert à nous sauver des illusions de la vérité instituée par la métaphysique. Mais, d'un autre côté, comme tous les phénomènes que la conscience représentative s'invente, la vérité est, elle aussi, de l'art. Les deux phénomènes, art et vérité, relèvent donc d'un seul et unique domaine, le domaine des apparences suscité par la volonté de puissance. Pour Nietzsche, c'est dire aussi que, par là, se trouve abolie toute distinction entre le monde vrai et le monde apparent. Le monde dévoilé par l'art n'est pas lui-même un monde vrai par opposition aux mondes des apparences. Inversement sortir du monde vrai c'est identiquement sortir du monde des apparences car les deux disent le même : ce ne sont que des valeurs établies par la volonté de puissance. C'est ce que Nietzsche indique d'une façon dont il faut bien comprendre le sens, dans le Crépuscule des idoles publié en 1889 :

Nous avons supprimé le monde vrai : quel monde reste-t-il alors? Le monde apparent peut-être? Mais non! Avec le monde vrai, nous avons aussi supprimé le monde des apparences! (OC VIII, p. 81).

On le voit, d'un côté, l'œuvre de Nietzsche ne s'affranchit certainement pas de la pensée métaphysique. La tâche y est toujours de penser la vérité de ce qui est en général et donc de référer le problème ontologique à la question de l'étantité de ce qui <est>. Dans l'optique de Heidegger, l'être continue ainsi d'être considéré par Nietzsche du point de vue seulement de l'étant. Autant dire que la question de l'être reste désertée par la pensée de Nietzsche, tout autant qu'elle a été désertée par toute la tradition. C'est ce que veut dire, pour Heidegger, que la pensée de Nietzsche participe donc encore au nihilisme européen. Mais, d'un autre côté, le nihilisme de Nietzsche devient absolu, car, à propos de la vérité de l'étant, il n'est désormais plus rien à dire qui excéderait le concept de valeur. Parce que la volonté de puissance ne connaît plus rien d'autre au-delà d'elle-même, s'éclipsent tous les leitmotive de la métaphysique traditionnelle comme les notions de vérité, de fondement et de présence absolue. Toutes ces notions sont désormais assujetties à la volonté de puissance où elles se liquéfient et sont finalement réduites à néant. Selon

${ }^{1}$ Mots manquants dans le texte original. (N. d. l'Éd.) 
Heidegger, la métaphysique arrive donc ici à son terme, puisque Nietzsche mène le nihilisme jusqu'à sa plus extrême conclusion par la négation de l'être au sens de la vérité de l'étant. Mais, toujours selon Heidegger, sans doute fallait-il que cette conclusion soit atteinte pour que puisse être atteinte en même temps l'essence véritable de la métaphysique dans ce nihilisme que Nietzsche ne fait que porter jusqu'à ses plus extrêmes aboutissants. Dans son Nietzsche, Heidegger écrit :

Pensé à partir de l'essence du nihilisme, le nihilisme surmonté dans le sens de Nietzsche n'est rien d'autre que le nihilisme accompli. Dans cet accomplissement, plus qu'en toute autre position fondamentale de la métaphysique, se révèle à nous la pleine essence du nihilisme (Nietzsche II, trad., p. 289).

Pour Heidegger, toutefois, si le stade du nihilisme accompli constitue le moment de cette extrême détresse de la pensée où toutes les illusions de la métaphysique sont perdues, ce stade est aussi celui où se dresse pour la pensée la possibilité d'accomplir le pas en arrière (Schritt zurück) de la métaphysique et d'en revenir à son oubli de l'être. Pour Heidegger, ce n'est pas encore la philosophie de Nietzsche qui accomplit ce pas en arrière, car son propre projet reste d'essence métaphysique bien qu'il mène la métaphysique à sa propre fin. Pour Heidegger, c'est plutôt à sa propre philosophie que revient d'avoir tenté ce pas en arrière de la métaphysique de façon à penser l'oubli de l'être qui est constitutif de la métaphysique elle-même et pour, ensuite, sortir de cet oubli, en posant enfin la question laissée pour compte par toute la métaphysique : qu'en est-il à proprement parler de l'être comme tel ? La seconde partie de ce cours sera donc employée à examiner comment Heidegger lui-même a tenté de répondre à cette question.

\section{Deuxième partie : L'ontologie fondamentale de Heidegger et le tournant linguistique}

Sans aucun doute possible, la question de l'être, ou la Seinsfrage, constitue la question essentielle de toute l'œuvre de Heidegger depuis Sein und Zeit en 1927 jusqu'aux derniers séminaires au Thor en Provence à la fin des années 60 et à Zähringen, près de Freiburg, en 1973.

Néanmoins, beaucoup de commentateurs de Heidegger ont souvent affirmé que Sein und Zeit et les premiers travaux de Heidegger se situaient dans une optique qui, sous le titre d'ontologie fondamentale, visait à accomplir le projet de la métaphysique plutôt qu'à le dépasser. Selon cette lecture, le 
dépassement de la métaphysique ne serait intervenu qu'ultérieurement, à l'époque dite du tournant ou de la Kehre et qui est dès lors souvent interprétée comme le lieu d'un tournant dans la pensée de Heidegger lui-même. Cette époque se situerait vers le milieu des années 30 et plus précisément peut-être dans l'essai sur L'Origine de l'œuvre d'art de 1935.

Il n'en reste pas moins que Heidegger lui-même s'est à plusieurs reprises opposé à cette interprétation de son propre itinéraire philosophique. Même à l'époque où le projet d'une ontologie fondamentale aura fait place à une méditation sur l'être, même donc à cette époque qu'il est convenu d'appeler le tournant, les quelques passages où Heidegger se livrera à une interprétation rétrospective de son propre chemin de pensée tendent tous à confirmer ce qu'il écrivait un jour à William Richardson :

La pensée du tournant provient de ce que je suis demeuré fidèle à la question qui était à penser dans Sein und Zeit (Cf. W. Richardson, Heidegger. Through Phenomenology to Thought, The Hague, <Nijhoff,> 1974, p. XVII ; trad. française de C. Roëls in Questions IV, <Paris, Gallimard, 1976,> p. 185).

Certes, on pourrait objecter que rester fidèle à une question ne veut pas dire nécessairement qu'on maintienne pour autant les thèses que l'on a défendues naguère en guise de réponses à la question. Mais, en ce qui concerne Heidegger, on peut également rétorquer que la continuité qu'il défend dans sa réponse à Richardson dit bien plus que le simple attachement à une même question, en l'occurrence la Seinsfrage. En effet, nulle part dans la suite de l'œuvre de Heidegger, on ne trouve d'éléments qui autoriseraient à dire qu'il ait été amené à revoir sa première réponse à la question de l'être. Autrement dit, jamais Heidegger ne remettra en cause les thèses essentielles de Sein und Zeit. Au contraire, il soutiendra toujours que l'ouvrage de 1927 représente déjà une rupture radicale d'avec la tradition métaphysique.

À propos de cette rupture, Heidegger sera très clair, par exemple, dans le dernier séminaire du Thor en 1969. Voici son explication. La métaphysique, dit-il, a toujours été une pensée de l'être en général et du temps, parce qu'il n'est pas possible de penser le premier sans penser le second. Mais, justement, la conception métaphysique du temps a toujours aussi été court-circuitée, parce que cette conception ne s'est jamais départie du privilège ontologique de la présence, comme si la seule dimension du temps qui prévalait pour penser l'être était la présence, et comme si être devait forcément vouloir dire être présent. À quoi Heidegger ajoute que c'est justement ce privilège de la présence qui est aboli dans Sein und Zeit, puisque l'analyse de l'existence de l'homme dont, dans cet ouvrage, Heidegger fait le fondement de la question 
de l'être, finit par déployer une temporalité sur le fondement de laquelle le sens de l'être devient compréhensible « en un sens non métaphysique », entendons autrement que comme présence (Questions IV, p. 273).

S'il arrive donc quelquefois à Heidegger de regretter que Sein und Zeit ait été écrit dans une langue qui était fort proche de la métaphysique dans la mesure où elle recourrait encore à des concepts comme celui de fondement, pour lui, cela n'affecte absolument pas l'éclaircie essentielle qui se produit dans l'ouvrage. À interpréter les propos de Heidegger lors du séminaire du Thor, on peut dire que l'éclaircie ici en question concerne l'analyse de la temporalité humaine, parce que, si elle doit servir de fondement à la question de l'être, ce fondement n'est plus une subjectivité qui assurerait la présence de tout ce qui est, mais c'est au contraire une existence radicalement finie sur le fondement de laquelle l'être ne se laisse plus dire en termes de présence.

Pour Heidegger, Sein und Zeit a donc été le premier pas dans la voie d'un dépassement de la métaphysique, et donc aussi le premier pas qui devait mener la pensée à s'ouvrir à un sens et à une vérité de l'être radicalement autres que celles qui ont prévalu dans toute la tradition métaphysique. En ce sens nouveau de l'être, venait à parler quelque chose comme une absence, et en ce sens nouveau de la vérité commençait à poindre quelque chose comme une retenue ou un retrait avec lequel apparaissait que la vérité de l'être ne se laisse pas réduire à la vérité de ce qui est présent. C'est bien la raison pour laquelle, au début des années 40, Heidegger pourra dire de Sein und Zeit qu' « il n'y a plus là aucune trace de métaphysique, mais un tout autre commencement » (Schelling $<$. Le traité de 1809 sur l'essence de la liberté humaine, trad. fr. J.F. Courtine, Paris, Gallimard, 1993,> p. 322).

Évidemment, cela ne veut pas dire que l'essence de l'être comme absence ou comme retrait soit déjà pensée dans Sein und Zeit. Cette pensée n'adviendra que huit ans plus tard, à partir sans doute du texte sur L'Origine de l'œuvre d'art. Mais, à suivre Heidegger lui-même, cette nouvelle tournure de la pensée ontologique ne représente pas une rupture, mais simplement un prolongement de l'ontologie fondamentale élaborée dans Sein und Zeit. Ce qu'il s'agira donc plus précisément de montrer ici, c'est que ce prolongement peut être interprété, ainsi que le suggérait Richard Rorty, comme une radicalisation linguistique de la thèse ontologique initiée par Heidegger en 1927. Ce qui nous oblige bien sûr à commencer par cette thèse ontologique en reprenant le projet d'ensemble de l'ontologie fondamentale de Sein und Zeit. 


\section{L'ontologie fondamentale de Sein und Zeit}

Si l'on se rappelle les indications de Heidegger lors du séminaire du Thor en 1969, la conquête essentielle de Sein und Zeit tiendrait dans le dévoilement de la temporalité de l'existence humaine. Selon Heidegger, c'est à partir de ce fondement qu'un pas décisif a été accompli qui devait permettre de comprendre le sens de l'être de façon non métaphysique. Aussi, tâchons d'éclairer cela en nous demandant d'abord de quoi il est essentiellement question dans Sein und Zeit. L'ouvrage de 1927 se présente lui-même comme un traité d'ontologie fondamentale. Ce qui veut dire deux choses.

Tout d'abord, comme traité d'ontologie, sa question essentielle et ultime concerne le sens de l'être. Elle peut être simplement formulée comme suit : qu'est-ce que l'être ? Selon Heidegger, répondre immédiatement à la question serait déjà court-circuiter le questionnement et c'est ce qu'a toujours fait l'ontologie traditionnelle qui, dès le départ, a préjugé de l'être en le confondant avec la présence. Plutôt que de procéder de la sorte, Heidegger propose donc de laisser la question ouverte et de faire de l'être l'objet d'une véritable question. Dans ce cas, tout ce qu'on peut en dire tient alors dans l'énigme suivante. Les choses sont tout autour de nous et, à tout moment, nous nous servons du terme « être » pour dire qu'elle sont ainsi présentes. Mais l'être luimême qui nous sert à dire la présence des choses n'est manifestement pas présent parmi les choses. À l'inverse du concept le plus général que nous avons des choses que nous nommons toutes des "étants », notre concept de l'être reste vide. Ce vide traduit simplement que l'être n'est pas quelque chose qui est parmi les choses qui sont, qu'il n'est pas non plus la somme de tous les étants ou quelque chose qui ressemblerait au monde présent qui nous fait face. L'être est tout simplement autre que ce qui est; il est autre que l'étant en général. Au départ, la question de l'être se justifie donc en raison de son énigme et cette énigme mérite d'être appelée celle de la différence ontologique. Voilà pour l'ontologie, c'est-à-dire pour la question essentielle et ultime de Sein und Zeit.

Mais, ensuite, cet ouvrage se présente également comme un traité d'ontologie fondamentale. En ce sens, sa question première doit être celle du fondement sur la base duquel il devient possible d'élucider la question essentielle et finale relative au sens de l'être. Or Heidegger indique très clairement que ce fondement ou ce sol est l'homme lui-même. Pourquoi l'être humain? Parce que celui-ci est cet étant qui se distingue de tous les autres étants du fait de sa compréhension. Parce qu'il comprend, l'être humain est donc assez naturellement le lieu de l'articulation du sens, non seulement du sens des étants et en particulier de l'étant qu'il est, mais aussi et principalement 
du sens de l'être lui-même en général. En d'autres termes, l'homme est le lieu où le sens de l'être doit pouvoir se laisser recueillir et saisir. Aussi, l'être humain est-il l'étant ontologique par excellence : il est le «là » de l'être, et c'est pourquoi Heidegger choisit de le nommer l'«être-là » ou le Dasein. Si c'est donc le Dasein humain qu'il s'agit d'interroger en premier lieu, cela veut dire que la question initiale de l'ontologie sera « qu'est-ce que l'homme ? » ou plus exactement « qu'est-ce que le Dasein ? », ou encore « que signifie l'être humain en tant qu'être-là ? ».

Devient donc assez compréhensible pourquoi Sein und Zeit consiste essentiellement en une analytique du Dasein. Par là, le but n'est bien sûr pas de proposer une anthropologie, mais de questionner le mode d'être spécifique de l'homme. Ce mode d'être, Heidegger le nomme « existence» et il précise ensuite le sens de l'existence en parlant d'elle en termes d' " être-au-monde » ou d'In-der-Welt-Sein. L'analyse du Dasein se présente par conséquent comme une analytique existentiale de l'homme visant à déterminer son mode d'être propre, c'est-à-dire à élucider le sens authentique et originaire de son être-au-monde. Ce qui est peut-être moins clair, ce sont les raisons pour lesquelles Heidegger commence cette analytique existentiale par la quotidienneté de l'homme ou par une description de son Alltäglichkeit. La question vaut d'autant plus d'être soulevée que ce point de départ a fait l'objet d'interprétations malencontreuses et, même, totalement erronées. Je pense en particulier à l'interprétation de Merleau-Ponty qui dans l'Avant-propos à sa Phénoménologie de la perception (1945) écrivait que :

Tout Sein und Zeit est sorti d'une indication de Husserl et n'est en somme qu'une explicitation du natürlicher Weltbegriff ou du Lebenswelt que Husserl, à la fin de sa vie, donnait pour thème premier de la phénoménologie (Phénoménologie de la perception, <Paris, Gallimard, 1945,> p. I).

À suivre une telle lecture, il semblerait que, si l'analytique existentiale du Dasein commence par emprunter les voies de la quotidienneté, ce ne peut être qu'en écho à l'idée husserlienne concernant la nécessité d'un retour au monde de la vie dans le but de saisir l'existence humaine comme telle, plutôt qu'à travers les concepts de la représentation scientifique ou métaphysique. Ainsi les philosophies de Heidegger et $<$ de $>$ Husserl auraient en commun un même souci qui caractériserait la démarche phénoménologique, à savoir en revenir à la vie ordinaire de telle façon que les concepts philosophiques puissent provenir d'un tel sol. Merleau-Ponty, exprimait encore une telle idée par exemple dans ce passage : 
La phénoménologie, c’est aussi une... philosophie qui replace les essences dans l'existence et ne pense pas qu'on puisse comprendre l'homme et le monde autrement qu'à partir de leur facticité... C'est aussi une philosophie pour laquelle le monde est toujours déjà là, avant la réflexion, comme une présence inaliénable, et dont tout l'effort est de retrouver ce contact naïf avec le monde pour lui donner enfin un statut philosophique (Phénoménologie de la perception, p. I).

Or il me semble que cette lecture est tout simplement fausse. En tout cas, elle ne permet pas une compréhension satisfaisante de l'analytique existentiale de Heidegger. Comme nous aurons l'occasion de le voir plus loin, en fait, le monde dont il est question dans le concept heideggérien d' " être-au-monde » ou d'In-der-Welt-sein n'a rien à voir avec le concept de Lebenswelt ou de « monde de la vie » chez Husserl. Les confondre ne peut donc mener qu'à des erreurs. À dire vrai, comme je voudrais à présent le montrer, les raisons pour lesquelles Heidegger commence par emprunter les voies de la quotidienneté sont diamétralement opposées à celles qui mènent Husserl à prôner un retour au monde de la vie, parce que, pour Heidegger, l'analyse du comportement ordinaire et quotidien de l'homme ne vise qu'à mettre en lumière une tendance contre laquelle il s'agit justement de prémunir la réflexion ontologique proprement dite. Expliquons-nous.

Comme nous l'avons vu, le projet ontologique de Heidegger est de tenter de se réapproprier la question essentielle de la métaphysique traditionnelle, tout en reconnaissant que, depuis le départ, la métaphysique a elle-même court-circuité la question concernant le sens de l'être. Selon Heidegger, ce court-circuitage relève en fait d'un préjugé ou d'un parti pris qui, pour lui, peut se résumer en un mot: la présence ou, en allemand, la Vorhandenheit. Toujours selon Heidegger, le préjugé en faveur de la Vorhandenheit se retrouve partout à la source des concepts fondamentaux de la métaphysique traditionnelle, ce qui montre comment celle-ci a toujours réglé la question de l'être avant même que de la poser. Aussi, dans son projet de réappropriation de la question de l'être, l'ontologie heideggérienne se présente elle-même comme une lutte contre la Vorhandenheit

Or d'où provient ce préjugé en faveur de la Vorhandenheit? Sur quel terrain commence-t-il toujours par s'imposer comme ce qui va de soi ? Selon Heidegger, ce terrain n'est pas d'abord celui de la philosophie, mais celui de la quotidienneté et c'est ce que Heidegger entreprend de montrer à travers une analyse des origines grecques du privilège de la Vorhandenheit. Pour Heidegger, tous les concepts essentiels de la philosophie antique sont profondément enracinés dans le champ de la vie quotidienne et, à ses yeux, la preuve 
la plus simple se trouve dans la conception courante que les Grecs avaient des choses en général, avant même l'apparition de leur conceptualisation philosophique. Comme nous le rappelle Heidegger dans Sein und Zeit, pour parler des choses en général, les grecs avaient un terme particulier: pragmata qui littéralement signifie ce à quoi l'on a affaire dans les occupations pratiques relevant de la praxis $\left(S Z\right.$, p. 68) ${ }^{1}$. Ce qui indique assez clairement que les Grecs comprenaient avant tout les choses non pas tellement comme de simples objets que l'on peut voir, mais plutôt comme ce dont on peut faire usage dans les activités les plus familières et les plus journalières. Ces activités consistent la plupart du temps d'abord à faire usage des choses disponibles et prêtes à l'emploi tout autour de nous ou à s'en servir afin d'en mener d'autres à la disponibilité moyennant des actes de production ou de fabrication.

Conformément à ce genre de comportement à l'égard des choses, la compréhension que nous en avons est donc avant tout une affaire de main. Son paradigme est celui de la manufacture, car nous comprenons les choses simplement telles qu'elles nous apparaissent lorsque nous les manipulons, nous en servons ou les façonnons. C'est très exactement de cette façon que les Grecs appréhendaient les choses en général. Aussi, à leurs yeux, les choses étaient d'abord telles qu'elles apparaissent comme du disponible, de l'utilisable ou du manipulable, bref comme ce qui est toujours, d'une manière ou d'une autre, à portée de la main ou sous la main. Selon une expression tout à fait courante en allemand, les choses étaient donc saisies par eux comme du vorhanden, c'està-dire comme ce qui est présent de telle façon que c'est disponible et qu'on peut donc en disposer ou tout simplement $<$ ' $>$ avoir. Ainsi, quand l'ontologie grecque aura à conceptualiser ce qui est ou l'étant au sens le plus large, elle aura assez naturellement recours à la manière quotidienne et ordinaire de concevoir les choses comme ce qui est sous la main.

Les Problèmes fondamentaux de la phénoménologie (Die Grundprobleme der Phänomenologie) est le titre d'un cours donné par Heidegger à Marbourg au semestre d'été 1927, donc l'année même de la parution de Sein und Zeit. Dans ce cours, Heidegger montre en détail comment tous les concepts fondamentaux de l'ontologie grecque découlent directement de la manière quotidienne de comprendre les choses en termes de disponibilité pour l'usage ou pour la fabrication. Parmi ces concepts, le plus important sans doute est

${ }^{1}$ La pagination indiquée est celle du texte original (Sein und Zeit, Tübingen, Niemeyer, 1927). L'auteur a traduit lui-même les citations en s'appuyant, dans certains cas, sur les traductions existantes (A. De Waelhens \& R Boehm, Paris, Gallimard, 1964 (partielle) ; E. Martineau, Authentica, 1985 ; F. Vezin, Paris, Gallimard, 1986). (N. d. l'Éd.) 
celui d'ousia, puisqu'il finira par s'imposer comme essentiel pour la conceptualisation philosophique de l'étant en général. Or, selon Heidegger, le terme ousia avait un sens originaire avant son usage théorique en philosophie mais que celui-ci va garder intact. À l'origine, en effet, ousia voulait dire la propriété, ce dont on dispose au sens des biens immobiliers comme la ferme et les terres. Identiquement il signifiait tout ce qui est là et dont $<$ on $>$ dispose à titre de propriété foncière ou de bien-fonds (Anwesen, en allemand, $<$ Die Grundprobleme der Phänomenologie (Sommersemester 1927),> GA XXIV, p. 153) ${ }^{1}$. Aussi, la généalogie du concept d'ousia, comme de tous les autres concepts ontologiques qui lui sont liés, montre que les philosophes grecs pensaient à la présence sous la main au sens de la disponibilité pour l'usage, lorsqu'ils tentèrent de conceptualiser la principale caractéristique de ce qui est au sens le plus général

Ainsi, on le voit, une conception de l'être formée sur ce mode spécifique qui, pour les choses en général, veut dire être sous la main ou être vorhanden dans le champ du comportement pratique de l'homme, a commencé de s'imposer en réduisant dès lors le sens général de l'être à la Vorhandenheit. Heidegger soutient ensuite que c'est cette conception qui se maintiendra dans toute la tradition philosophique, même quand, par la suite, toute référence explicite à la pratique quotidienne aura disparue. Ainsi, que ce soit au sens de l'existentia ou de l'actualitas médiévale ou que ce soit au sens moderne de la Wirklichkeit ou du Sein, le mot « être » continuera de signifier être-sous-lamain ou être présent, fût-ce sur ce mode affadi qui veut simplement dire : se trouver là comme quelque chose d'effectif et, comme tel, produire de l'effet sur notre réceptivité perceptive.

Telle apparaît donc la thèse centrale de la lecture heideggérienne des Grecs à l'époque de Sein und Zeit: en amont de toute la tradition philosophique, la pensée grecque montre, par l'origine de ses propres concepts, le sol d'où est née est la tendance à comprendre l'être comme présence ou comme Vorhandenheit. Ce sol est celui de la vie active quotidienne et c'est le sillage de la quotidienneté qu'a toujours suivi la théorie philosophique, ne faisant simplement que prolonger la façon de voir du comportement ordinaire, même si c'est sans en avoir eu une conscience explicite.

Or quelle est précisément la façon de penser et de voir de l'attitude quotidienne qui est aussi implicitement la conception de la métaphysique traditionnelle en tant que métaphysique de la présence ou de la Vorhandenheit ? Selon les descriptions très détaillées de Heidegger, cette attitude est celle

${ }^{1}$ Le sigle « GA » renvoie à M. Heidegger, Gesamtausgabe, Frankfurt a. M., Klostermann, 1975 suiv. (N. d. l'Éd.) 
qui, à tout moment de son activité journalière, tient le regard de l'homme rivé sur les objets de sa préoccupation et le rend ainsi entièrement absorbé par tout ce à quoi il a constamment affaire : non seulement les réalités du monde, mais, d'une façon plus large, le monde lui-même. Ce monde qui concerne notre vie quotidienne, Heidegger l'appelle l'environnement ou le monde environnant, l'Umwelt. Il constitue la proximité tout autour de nous où, en relation les unes avec les autres, les choses font immédiatement sens pour qui agit sur elles et est occupé par elles. Toujours solidaire des mains, le regard du comportement quotidien n'a d'yeux que pour ce monde sur lequel l'homme focalise constamment son attention en chacune de ses intentions ou en chacun de ses projets. Ce regard propre à l'attitude quotidienne est parfois nommé par Heidegger la circonspection pratique ou la praktische Umsicht. De celle-ci se dégage un sentiment d'attachement profond au monde, comme s'il était le lieu d'irréfutables évidences qui régulent constamment le bon déroulement de nos expériences et justifient donc qu'on accorde au monde une confiance sans limite. Aussi est-ce dans ce monde que l'homme reconnaît avoir son chez-soi.

Comprenons bien dès lors tout ce qu'implique cet attachement au monde qui est une caractéristique fondamentale de l'attitude quotidienne de l'homme et qui détermine la compréhension la plus immédiate qu'il a de luimême aussi bien que de tout ce qu'il n'est pas. Les choses et les autres hommes sont immédiatement compris quotidiennement comme ce à proximité de quoi et avec qui nous séjournons dans le monde. C'est pourquoi nous vivons habituellement avec la conviction ou la ferme croyance d'être les habitants d'un monde qui est familier et où notre principale préoccupation est de construire et de parfaire les multiples formes de notre habitat parmi les choses et avec les autres. Le monde environnant ou l'Umwelt prend dès lors les apparences d'une présence en fonction de laquelle non seulement nous comprenons le sens des choses et des autres, mais comprenons aussi le sens de notre propre existence. Tout commerce avec les choses, toute communication avec les autres et donc aussi toute communion avec soi-même présuppose, comme sa condition de possibilité, l'adhésion originaire à cet horizon de présence qui procure son sens à tout ce qui est, y compris nous-mêmes, et nous tient toujours dans cette évidence. Aussi ce qu'on nomme l'évidence naturelle, la natürliche Selbstverständlichkeit, ne veut rien dire d'autre que la croyance et la confiance premières en cette commune présence qu'est le monde et où semble être fixé le «là » de toute réalité, aussi bien que le «là » de notre propre être-là. De cette façon, toute existence, la nôtre y compris, semble confondue dans une symbiose mondaine unique et indifférenciée. D'où apparaît également que ce monde, qui nous tient lieu de certitude absolue, est essentiellement un monde commun. Dit autrement, c'est un monde dans le champ duquel nous ne faisons 
nous-mêmes que comparaître parmi les autres étants. De cette façon, aussi bien pour cet étant que nous sommes que pour tous les étants que nous ne sommes pas, être ou exister veut essentiellement dire prendre part, de la même façon, à la vie du tout. C'est aussi la raison pour laquelle, les affirmations relatives au je suis prennent bien souvent la forme du on est et que le on passe ainsi pour le véritable sujet des phrases quotidiennes. C'est là, somme toute, le signe que chacune des expériences quotidiennes se produit avec la conscience profonde de notre participation à la communauté du monde. Ce genre de conscience pourrait très bien servir à expliciter ce qu'on appelle le « sens commun ».

Or, pour Heidegger, c'est justement parce que l'homme s'en tient au sens commun qu'il n'a pas de compréhension réellement adéquate de sa propre existence. Au contraire, le sens commun tend toujours à diluer la signification de l'existence humaine dans la commune présence qui fait le monde. Aussi, telle que l'homme la comprend dans la quotidienneté, son existence signifie être au monde. Mais, ici, être au monde signifie seulement habiter le monde, séjourner en lui parmi les choses et avec les autres. Quotidiennement parlant, se comprendre soi-même, c'est ressentir son être comme être présent dans le monde.

Aussi Heidegger soutient-il que ce mode habituel de l'autocompréhension revient à ne faire aucune différence entre son propre mode d'être et le mode d'être de chaque chose à laquelle nous avons affaire dans notre comportement quotidien. En d'autres termes, la compréhension quotidienne de soi promeut l'indifférence ontologique en réduisant tout mode d'être au seul mode d'être de la présence et donc en nous dérobant la plupart du temps à nous-mêmes la spécificité de notre propre existence. C'est pourquoi Heidegger qualifie d'inauthentique l'auto-compréhension quotidienne. Dans son esprit, cela ne signifie pas que la compréhension quotidienne n'atteindrait pas encore notre existence comme telle. Au contraire, l'inauthenticité est un mode d'être réel de notre existence qui, au quotidien, consiste à se détourner de soimême pour comprendre notre être-là à partir de la présence mondaine. L'inauthenticité doit donc être considérée comme une dimension réelle de notre existence : elle est quelque chose qui appartient à l'histoire de l'homme. En d'autres termes, elle est un existential, une structure fondamentale de son être-là, et, plus précisément encore, cet existential qui doit être appelé la déchéance ou le Verfall. Par ce mot, nous voici donc fixés sur le véritable statut de la quotidienneté dans l'ontologie fondamentale de Heidegger: la vie quotidienne est ce mode d'être qui, pour notre être-là, consiste à décliner sa propre spécificité, à se fuir soi-même pour un monde où il commence toujours par abandonner le véritable sens de son existence. Comme Heidegger le dit 
dans un cours du semestre d'été 1925 , la déchéance qui caractérise la quotidienneté équivaut à un véritable phénomène d'apostasie (Abfall) existentiale ( $<$ Prolegomena zur Geschichte des Zeitbegriffs (Sommersemester 1925), > GA XX, p. 390-391) ou de renonciation à soi-même.

Les raisons pour lesquelles l'analytique existentiale de Sein und Zeit commence par le comportement quotidien sont donc à présent suffisamment claires et ne sauraient plus longtemps nous abuser. Bien loin de faire écho au projet phénoménologique husserlien d'un retour à la Lebenswelt, comme on les a souvent présentées à la suite des indications erronées de Merleau-Ponty, les analyses que Heidegger consacre au comportement ordinaire ne visent qu'à porter au jour la prégnance d'une tendance contre laquelle il s'agit précisément de prémunir la méditation ontologique proprement dite. Cette tendance inhérente à la compréhension quotidienne est celle que le concept de déchéance veut signifier : en raison de la pression constante des choses dans la vie de tous les jours et de la fascination pour le monde qui en résulte, cette tendance consiste à se comprendre habituellement soi-même à partir de ce qu'on n'est pas et à accorder ainsi la préséance ontologique à un mode d'être qui ne saurait nous appartenir authentiquement.

Comme Heidegger le soutient aussi, cette tendance est si profondément enracinée dans les habitudes les plus naturelles de l'homme qu'elle a tôt fait d'orienter la conceptualisation théorique de l'être et de déterminer ainsi toute l'histoire de la philosophie occidentale. Comme nous l'avons vu, on peut en trouver la preuve dans la manière dont la présence sous la main ou la Vorhandenheit a prévalu, depuis le départ, dans l'ontologie grecque. Cela montre clairement que la question ontologique n'a jamais été traitée autrement que d'après le mode inauthentique de la compréhension quotidienne.

Certes, la philosophie se doit d'être réflexive, au sens où elle doit chercher à appréhender et à concevoir l'être, en référence à cet étant qui, de façon générale, comprend et qui est l'homme en tant que Dasein ou être-là. Aussi, l'ontologie grecque a cherché à concevoir l'être en général par réflexion sur le logos ou la psychè qui étaient, pour elle, autant de façons d'exprimer le Dasein humain. Mais parce que cette nécessaire référence à l'homme n'a précisément jamais dépassé le niveau de son auto-compréhension quotidienne en tant que simple habitant ou être-là dans le monde, l'ontologie grecque n'at-elle jamais fait que cautionner le sens de l'être qui ainsi prévalait. Pour Heidegger, telle est la naïveté dont s'est dès le départ rendue coupable l'ontologie grecque en maintenant la réflexion philosophique dans les ornières de la vie quotidienne et donc sous la tutelle du mode déchéant de la compréhension de soi. Dans le cours du semestre d'été 1927, Heidegger écrit : 
L'ontologie n'est pas naïve au sens où elle ne se tourne pas vers le Dasein et n'est donc pas réfléchie - cela est exclu —, mais dans la mesure où cette nécessaire rétro-spection en direction du Dasein ne dépasse pas une conception vulgaire du Dasein et de ses attitudes, et par conséquent ne le fait pas véritablement ressortir, dans la mesure où précisément elle appartient à la quotidienneté du Dasein en général. Ainsi la réflexion reste-t-elle prise dans les voies de la connaissance pré-philosophique ( $<$ Die Grundprobleme der Phänomenologie (Sommersemester 1927),> GA XXIV, p. 155-156).

Or, comme Heidegger le montre également, il serait vain de vouloir trouver dans la philosophie moderne quelque amendement à la naïveté de l'ontologie ancienne. Nonobstant sa ferme intention de fonder l'ontologie par retour à la spécificité de l'ego, le prétendu renouveau de la philosophie moderne trahit une naïveté non moins grande lorsque, afin d'identifier cet ego, elle recourt, par exemple, à la notion de res cogitans par opposition à celle de res extensa ou lorsqu'elle recourt à la notion de « chose ayant sa fin en soi » par opposition aux « choses qui ne sont que simples moyens », ou encore lorsqu'elle recourt à la notion de sujet par opposition à celle d'objet. En effet, il est aisé de voir que toutes ces oppositions à travers lesquelles la pensée moderne prétend cerner $<$ la $>$ spécificité du Dasein humain sont articulées autour d'un concept indifférencié de l'être dont la trame substantialiste et choséiste (res, Ding, subjet) montre à l'évidence qu'il n'est pas forgé d'après le mode d'être authentique de l'homme, mais, au contraire, d'après un mode d'être qui lui est impropre, puisqu'il provient de la réalité qu'il n'est pas. Ici aussi la conception de l'être est déjà confisquée par l'idée de présence comme Vorhandenheit et relève d'un parti pris dont les plus lointaines origines sont l'accaparement, l'absorption et finalement la fascination quotidiennes par ce monde des réalités tout autour de nous.

Il s'ensuit assez naturellement que c'est au dépassement des effets conceptuels de cette absorption quotidienne dans le monde que Heidegger consacre tous ses efforts dans son propre projet d'une ontologie fondamentale. Parce que ce projet requiert nécessairement la question de l'homme en tant qu'il est cet étant qui seul existe sur le mode de la compréhension et donc abrite en son être propre le sens de l'être en général, à son tour cette question requiert de l'homme lui-même la clairvoyance et la perspicacité que précisément lui interdit son éblouissement quotidien par la présence. Telle que l'entend Heidegger, cette clairvoyance n'est autre que la capacité de distinguer son propre mode d'être par rapport au mode d'être qui ne pourrait être le sien en propre. En d'autres termes, il s'agit de parvenir au discernement qui chaque fois nous fait défaut aussi longtemps que nous sommes absorbés par tout ce qui nous est proche, de telle façon que nous ne comprenons notre existence 
que par rattachement au monde, n'ayant d'autre conception de ce monde que celle de la présence environnante. Identiquement, ce discernement est aussi ce qui manque à l'ontologie traditionnelle, lorsque, dans le prolongement de la tendance quotidienne, elle souscrit d'emblée à une acception indifférenciée de l'être qui, pour tout étant, signifie être présent. À l'inverse d'une telle confusion, l'ontologie fondamentale de Heidegger exige d'abord que soit faite la différence du propre et de l'impropre. Elle demande donc de commencer par distinguer entre notre propre manière d'être-là et la manière d'être-là qui ne pourrait être la nôtre en propre. En d'autres mots encore, la tâche ontologique première est de tenter de conjurer le mauvais sort existential qui, quotidiennement, nous condamne à exister de manière inauthentique. Seule l'éviction de la déchéance quotidienne semble donc susceptible d'ouvrir la voie à la question de l'être-là propre de l'homme et, par là, à la question de l'être comme tel.

Or, une des thèses principales de Heidegger dans Sein und Zeit est de soutenir que c'est à travers le phénomène de l'angoisse que s'ouvre pour l'étant que nous sommes la possibilité de dépasser sa déchéance quotidienne. L'angoisse, écrit Heidegger, est ce sentiment de situation tout à fait particulier, car lorsqu'elle se produit :

Elle enlève au Dasein toute possibilité de se comprendre de manière déchéante à partir de son monde et de l'interprétation commune. Elle rejette le Dasein vers ce pourquoi il s'angoisse, vers son pouvoir-être-au-monde le plus propre (SZ, p. 187).

À partir de ces lignes, tâchons donc de cerner au plus près la charge phénoménale de l'angoisse avec laquelle, comme on peut l'entendre, semble s'annoncer le sens authentique de l'existence ou de l'être-au-monde de notre être-là.

Parmi tous les phénomènes qui sont susceptibles de nous mettre face à notre existence, l'analytique existentiale de Sein und Zeit réserve une place spéciale aux humeurs ou aux sentiments, c'est-à-dire au domaine de la Stimmung en général. Comme l'explique Heidegger au $§ 29$ de l'ouvrage, la raison en est que les humeurs ne sont pas de simples états d'esprit comme les autres, mais constituent plus spécialement la manière la plus simple et la plus primitive que nous avons d'éprouver notre situation dans le monde. En d'autres mots, les humeurs ont le pouvoir ontologique de nous mettre face à notre propre facticité ou à notre propre situation dans le monde et de nous révéler notre propre existence comme être au monde. Néanmoins, tel qu'il se produit quotidiennement, le sentiment de cette situation dans le monde est le 
plus souvent obnubilé par la présence des choses et des autres qui précisément nous mettent de telle ou telle humeur. Ainsi, plutôt qu'à partir de nous-mêmes, c'est à partir de tout ce que nous ne sommes pas qu'est ordinairement éprouvée et ressentie notre situation dans le monde. Ce qui veut dire que, ordinairement, nos affects et nos humeurs nous manifestent notre situation dans le monde sur un mode qui demeure un mode déchéant.

Mais, selon Heidegger, tel n'est pas le cas de l'angoisse qui, elle, ne connait justement pas la médiation des choses ou des autres. Aussi, montre Heidegger, pour comprendre ce qu'est l'angoisse, il faut d'abord clairement la distinguer de la peur. La peur est un sentiment qui ne se produit qu'à l'approche d'un étant dont la menace est telle qu'elle nous dévoile subitement notre existence sur le mode de l'être menacé. Dans l'angoisse, au contraire, il n'est absolument rien d'extérieur à nous-mêmes qui pourrait servir à expliquer pourquoi notre être-là s'angoisse. À la différence de la peur, l'angoisse ne se produit pas à l'approche d'un étant déterminé. Cette indétermination de ce qui nous angoisse ne provient pas d'une hésitation à en préciser l'origine, mais elle montre plutôt que rien de ce qui est dans le monde, aucun étant autour de nous, ne pourrait servir à dire ce face à quoi nous éprouvons de l'angoisse. Si celleci nous laisse sans voix, c'est que plus aucune signification ordinaire se référant aux choses dans le monde ne peut encore servir à exprimer l'origine de notre angoisse. Se dresse dès lors la question : qu'est-ce qui, à proprement parler nous prend par la gorge dans l'angoisse, si ce n'est rien de présent au sens que nous prêtons ordinairement à ce mot, s'il s'agit donc de quelque chose qui doit bien être là sans être nulle part? À cette question, la réponse de Heidegger est la suivante : ce qui nous tient dans les tenailles de l'angoisse, c'est le monde comme tel, mais un monde précisément expurgé de toute sa signification habituelle et commune. Il ne s'agit donc pas du monde quotidien de la présence, mais du monde de notre situation originaire, du monde qui appartient ontologiquement et essentiellement à la condition la plus propre de notre être-là en tant qu'être-au-monde. Comme le dit Heidegger :

Si c'est donc le «rien », c'est-à-dire le monde comme tel, qui se montre luimême comme le face à quoi de l'angoisse, cela signifie que ce devant quoi s'angoisse l'angoisse est l'être-au-monde lui-même (SZ, p. 187).

C'est donc pourquoi Heidegger nomme l'angoisse un sentiment fondamental de situation, une Grundstimmung. L'angoisse a précisément ceci de fondamental qu'elle est un sentiment que nous éprouvons non pas face aux choses du monde ambiant, mais bien plutôt face à notre propre situation dans le monde. C'est cette situation qui nous concerne en propre ou notre propre être-au- 
monde qui nous est ainsi révélé et cette révélation se produit toujours dans l'angoisse.

Le point important est ici que le monde familier n'est désormais plus d'aucun secours pour la compréhension de notre condition propre d'existence. Lorsque l'angoisse nous submerge, le monde environnant quotidien disparaît et, avec lui, les étants du monde s'effacent soudainement comme si tous désormais n'étaient plus d'aucun poids, mais au contraire totalement inconsistants et sans importance. Ainsi l'angoisse barre la voie à la propension habituelle que nous avons de comprendre notre existence en fonction de la présence des choses et des autres qui nous préoccupent et nous sollicitent dans la vie de tous les jours. Autrement dit, la façon quotidienne de se comprendre soi-même s'interrompt avec l'angoisse et, selon Heidegger, c'est précisément à la faveur de cette interruption que l'homme peut désormais faire face à sa propre existence comme à quelque chose qui ne se laisse plus comprendre à partir de ce qu'il n'est pas. Aussi faut-il dire que l'angoisse isole notre être-là et que cet isolement par rapport à tout ce dont est fait le monde de la vie quotidienne nous mène au-devant de notre condition d'existence la plus propre, face donc à nous-mêmes en tant qu'être-au-monde.

Aussi, comme nous l'avons déjà dit, l'angoisse est bien plus qu'un simple épisode de l'existence de notre être-là. Elle y occupe au contraire une place cardinale, puisque, lorsqu'elle survient, l'angoisse rompt tous les liens qui habituellement nous rattachent au monde de la présence. De cette façon, l'angoisse nous défait de la tendance quotidienne à nous comprendre nousmêmes à partir de ce qui est présent. Ce qui signifie aussi que l'angoisse prend place dans notre existence comme un événement qui, pour quelques instants, interrompt le sort qui habituellement nous condamne à la déchéance.

Aussi est-ce la déchéance elle-même, qui s'éclaire de façon nouvelle à la lumière du phénomène de l'angoisse. Comme nous l'avons vu, la déchéance n'est jamais qu'une fuite de soi-même en direction du monde commun des choses et des autres. Mais, justement, la raison d'une telle fuite n'est-elle pas que ce monde se présente toujours à notre existence comme un refuge où il semble apaisant de se sentir en intimité avec les choses et les autres personnes ? Adhérer au monde environnant est, pour nous, la meilleure façon d'éluder notre propre condition dont les signes inquiétants se manifestent dans l'angoisse. Se sentir solidaire du monde n'est que la façon dont <nous> esquivons la plupart du temps notre propre condition d'être-au-monde. Or lorsque l'angoisse nous retranche de tout dans l'isolement, elle nous révèle justement que notre condition d'être-au-monde est de n'avoir pas de chez-soi dans le monde. En d'autres mots, l'angoisse est angoisse parce qu'elle nous 
confronte à notre propre extranéité ou à ce que Heidegger nomme 1'Unheimlichkeit. Elle seule peut traduire le véritable statut de notre existence et c'est cette révélation inquiétante qui nous est faite et ne peut nous être faite que dans l'angoisse, puisque justement elle nous révèle de nous-mêmes que nous sommes des étrangers à ce monde et n'y avons donc pas notre chez-soi.

Le sans-chez-soi (das Un-zuhause) doit être conçu d'un point de vue ontologique et existential comme le phénomène plus originaire (SZ, p. 189).

Il apparaît finalement que c'est ce statut d'étranger sans véritable domicile en ce monde - ce que signifie très exactement l'Unheimlichkeit - que nous cherchons continuellement à esquiver et à nous cacher à nous-mêmes en tâchant au contraire de trouver dans le monde, auprès des choses et des autres, le réconfort d'un chez-soi. Tandis que nous adhérons quotidiennement au monde serein et apaisant de la présence, que nous fusionnons avec toutes les entités intramondaines et nous comprenons nous-mêmes à partir d'elles, nous ne cherchons qu'à fuir la condition angoissante de notre être-au-monde qui est d'être sans chez-soi en ce monde. À mieux y regarder, l'attraction quotidienne pour la présence au sens de la Vorhandenheit est toujours plus fondamentalement une volonté de refouler et d'occulter à soi-même son extranéité la plus propre. Tel est bien ce que semble vouloir dire Heidegger quand il écrit :

La fuite déchéante (Die verfallende Flucht) vers le chez-soi du commun est une fuite devant le sans-chez-soi (vor dem Unzuhause), c'est-à-dire devant l'extranéité (Unheimlichkeit) qui se trouve au cœur même de l'être-là en tant qu'êtreau-monde jeté et remis à lui-même en son être (SZ, p. 189).

Mais, au juste, comment comprendre que, telle qu'elle nous est révélée dans l'angoisse, notre l'existence est fondamentalement sans chez-soi dans le monde ? Je pense que Heidegger répond à cette question lorsqu'il détermine de façon plus précise ce qu'a justement d'angoissant la révélation du mode propre de notre être-au-monde. Il écrit à ce propos :

Ce devant quoi (Wofor) il y a l'angoisse c'est l'être-au-monde dans son être jeté (das geworfene In-der-Welt-sein), ce pour quoi (Worum) il y a angoisse est le pouvoir-être-au-monde (In-der-Welt-sein-können) (SZ, p. 191).

Que signifie cela ? Tout d'abord, comme le dit assez clairement le texte, ce qui se révèle de façon angoissante à propos de notre propre existence est sa déréliction ou son être jeté (Geworfenheit). Par ce terme, Heidegger veut exprimer la facticité (Faktizität) de notre existence (SZ, p. 135). Mais cette 
facticité est tout autre chose que la simple factualité sur laquelle la compréhension ordinaire et commune rabat la première. En effet, la compréhension ordinaire ne distingue pas la situation de l'homme dans le monde par rapport à tout ce qui s'y trouve présent de fait. Ce qui, au contraire, se révèle dans l'angoisse est que la situation de l'existence est d'avoir été jetée dans un monde à la présence duquel nous ne saurions rester attachés sans perdre de vue le seul projet, le seul pouvoir-être auquel nous sommes destinés du fait d'avoir été versé dans le monde. Mais quel est justement ce projet le plus propre de notre existence qui ne se révèle que dans l'angoisse ?

Pour répondre à cette question, il n'est que de considérer tout d'abord la manière par laquelle nous comprenons quotidiennement les projets et les potentialités de notre existence. Deux traits intimement liés caractérisent ce que signifient habituellement nos possibilités. Le premier de ces deux traits tient à la réalisation. En effet, dans l'ordre de nos préoccupations quotidiennes, toutes les possibilités sur lesquelles nous nous projetons sont toujours liées aux choses du monde qui nous procurent quelque chose à réaliser. De cette relativité à l'intramondain résulte donc que chacune de nos ouvertures à un possible consiste soit à le réaliser, soit à guetter le moment propice à sa réalisation. Dans un cas comme dans l'autre, nous faisons en sorte que le possible perde son caractère de possible pour être réalisé de façon à ce qu'ainsi quelque chose devienne présent qui ne l'était pas encore. Mais ce premier trait en engendre un second. Il est assez évident, en effet, que les possibilités qui tiennent quotidiennement notre existence en haleine s'ouvrent en un champ infini. Aussi, grande consommatrice de possibles, l'existence quotidienne ne se satisfait jamais d'aucune de leurs réalisations. Cette insatiabilité qui quotidiennement maintient l'être-là sur le mode du projet fait en sorte qu'il est un étant qui n'en a jamais fini ou qui plutôt n'est jamais fini. Or ce perpétuel inachèvement du projet existential a pour conséquence que, à aucun moment de l'expérience quotidienne, une possibilité ne pourrait se présenter qui coïncide avec la dé-finition de l'existence humaine et de son véritable projet. $\mathrm{Au}$ contraire, étant quotidiennement disséminé dans la multitude des possibilités qui s'offrent à lui, l'homme n'en maîtrise aucune dans laquelle il pourrait saisir le véritable sens de son propre pouvoir-être, le sens de son projet authentique dans le monde.

S'il doit donc y avoir une possibilité d'être avec laquelle pourrait être saisissable le sens unitaire de notre existence, il faut que ce soit une possibilité indépassable de notre existence, c'est-à-dire une possibilité dont le projet mène à nous comprendre nous-mêmes, de façon unitaire, dans notre propre définition, ce qui veut dire aussi notre propre achèvement ou notre propre fin. En outre, parce que cette possibilité doit donc être indépassable, elle ne saurait 
être non plus de l'ordre de toutes celles qui, quotidiennement, se prêtent à la réalisation. Au contraire, il doit s'agir d'une possibilité qui, ne donnant précisément rien à réaliser, se préserve elle-même dans sa pure possibilité. Cette pure possibilité, dont l'actualisation est toujours en retrait et dont le projet dévoile pourtant l'existence à elle-même en la ramassant en sa propre définition, ne peut avoir qu'un nom dans l'analytique existentiale de Heidegger : c'est la mort.

Bien sûr, il ne s'agit <pas > ici de cette mort anonyme qui est relative aux autres et qui, comme telle, appartient elle-même au champ de la présence quotidienne. Il s'agit plutôt de la mort qui est sans aucun rapport avec celle des autres mais qui, ne concernant donc que nous-mêmes, nous est à ce point certaine qu'elle constitue une vérité bien plus radicale que toutes les affirmations habituelles concernant notre être. Telle est, en définitive, l'unique certitude que nous sommes appelés à reconnaître dans la solitude de l'angoisse, tant il est vrai que la compréhension de notre existence comme être voué à la mort (Sein zum Tode) est essentiellement angoisse et que fondamentalement celle-ci ne peut donc être qu'angoisse face à l'imminence de la mort qui irrémédiablement promet notre possibilité d'être la plus propre à l'impossibilité pure et simple. Identiquement, c'est aussi avec notre mort propre que s'éclaire notre véritable facticité dans le monde : nous y avons été jetés dans le seul et unique projet de la mort :

Comme sentiment fondamental de l'être-là, écrit Heidegger, l'angoisse est la découverte de ce que l'être-là, en tant qu'être jeté, existe en vue de sa fin (SZ, p. 251).

Parce que la mort est elle-même présente dans le monde quotidien, elle ne nous est jamais totalement méconnue. Toutefois, montre Heidegger, cette appréhension est toujours aussi une façon de se cacher à soi-même la véritable signification de la mort en n'y voyant qu'un événement parmi d'autres. Cet événement ne semble pas nous toucher en propre et, même quand la mort de l'autre nous plonge dans un profond chagrin, ce sentiment n'est jamais assez profond pour résister à la conviction rassurante que la vie ne saurait tarder à reprendre le dessus. Cette minimisation de la mort et cette confiance éperdue dans la vie sont simplement le voile que nous jetons d'habitude sur la vocation de mort qui marque notre existence comme son caractère le plus propre et qui, du même coup, rend illusoire notre demeure en ce monde. À mieux y regarder, pourtant, notre engouement ordinaire pour les choses et le monde est lui-même déterminé par notre propre vocation de mort. Même jusque dans la fuite qui consiste à se divertir de notre être-pour-la-mort au gré des affairements de la 
vie quotidienne, la mort domine le tableau de notre existence. Elle se profile comme l'unique souci de notre être-là et s'éclaire même comme ce que nous cherchons à décliner dans l'insouciance des préoccupations quotidiennes.

Il est dès lors assez facile de comprendre pourquoi, d'après Heidegger, la différence entre le mode authentique et le mode inauthentique de notre existence ne dépend que de notre attitude à l'égard de la mort. Si l'inauthenticité est le principale caractéristique de l'attirance quotidienne pour le monde en tant qu'elle est la façon commune d'éluder notre être-pour-la-mort, en revanche l'authenticité ne saurait consister qu'en sa prise en charge. L'être-là n'existe donc authentiquement que dans l'assomption de sa propre mortalité. C'est seulement en faisant droit à la condition la plus propre de son existence comme être-au-monde que l'authenticité équivaut simplement à la résolution pour soi-même.

Mais, dans de telles conditions, on ne peut que se demander quelle place le mode authentique de notre être-au-monde peut encore accorder au monde lui-même. Comme nous l'avons vu, cette authenticité ne relève finalement que de notre entière réceptivité à l'annonce qui se produit dans l'angoisse. Comme le dit Heidegger, prendre résolution pour soi-même n'est qu'une affaire de capacité d'écoute à l'appel ou au rappel angoissant de l'existence. Dans Sein und Zeit, Heidegger identifie cet appel à « la voix de la conscience » (Stimme des Gewissens) (SZ, p. 268). Cet appel qui s'élève de nous-mêmes n'est que le rappel à nous-mêmes de la condition la plus propre de notre existence. Quant à la condition existentiale que nous nous intimons de reconnaitre en prêtant une écoute à cet appel, c'est d'avoir été jeté dans un monde auprès duquel nous ne saurions nous attarder parce que nous nous pressons vers la mort. D'où le sentiment d'angoisse et de solitude dont forcément se nimbe cette révélation, puisque, en assumant cette condition la plus propre de notre existence, nous nous découvrons comme en terre étrangère; une terre où nous ne pourrions donc trouver la quiétude d'un chez-soi sans précisément y perdre le sens authentique de notre existence. Dans ce monde, faut-il le dire, l'existence authentique est sans chez-soi, Un-zuhause. Sa vraie condition est d'être-aumonde sans avoir la possibilité d'habiter authentiquement en lui.

Pour qui ose donc l'épreuve de soi dans l'isolement de l'angoisse, et existe ainsi dans la résolution pour soi-même, il n'y a plus aucune lumière provenant de l'extérieur qui pourrait encore tant soit peu éclairer le sens véritable de l'existence. Avec le dévoilement de l'extranéité comme fondamentalement constitutive de notre existence, tous les signes à partir desquels nous nous comprenons d'ordinaire comme les habitants et les cohabitants du monde tombent en désuétude. En fait, en regard de notre être-au-monde authentique, c'est tout simplement le monde qui sombre dans la plus totale 
nullité. Il reste alors seulement à notre être-là d'être confronté à la nudité de sa propre existence dans l'inanité du monde. Aussi, lorsque l'être-là humain est réveillé de sa déchéance ordinaire par l'appel de sa conscience, il comprend que l'appelant n'est autre que son propre être-là, mais qu'il s'agit désormais de

l'être-là en son extranéité, il est l'être-au-monde originellement jeté en tant que sans-chez-soi, il est $<$ le « que »> nu dans le rien du monde (das nackte „Dass“ im Nichts der Welt) (SZ, p. 276-277).

Pour Heidegger, il n'y a donc de dévoilement de soi en son être propre qu'au sens d'un tel dénuement. Ce dévoilement est donc toujours essentiellement celui d'une existence que plus rien ne saurait voiler, car plus rien d'extérieur ne pourrait encore la déterminer en dehors de sa propriété la plus propre, à savoir son processus ou sa dynamique propres. Exprimée dans les termes de Sein und Zeit, cette dynamique consiste à avoir été jeté dans un monde où nous nous projetons vers notre mort, et ce qui nous rend cette dynamique existentiale conceptuellement concevable, ce qui en constitue donc le schème, c'est le temps. Non pas, bien sûr, le temps que la compréhension quotidienne ramène à la succession de maintenant et qui demeure la conception du temps de la philosophie traditionnelle depuis Aristote. Cette définition du temps comme temps des horloges ne nous concerne pas en ce que nous avons de propre, parce qu'elle est seulement relative au mouvement naturel des choses dans le monde. À l'inverse de cette conception déchue, le temps qui est ici en question est la temporalité existentiale authentique selon laquelle nous n'existons que tendus vers l'avenir de notre fin à partir du passé de notre avoirété-jété dans le monde.

Il apparaît très nettement désormais que, entre ce futur et ce passé authentiques, il ne pourrait y avoir de place pour le présent chez Heidegger. Le présent n'a tout simplement pas de consistance dans l'existence authentique. Seule au contraire lui correspond la déchéance, c'est-à-dire le mode inauthentique de notre être-là. Certes, Heidegger nous parle bien d'un présent authentique qu'il nomme l'Augenblick, c'est-à-dire l'instant de la vision concernant la vérité de notre existence. Comme tel, ce présent est donc seulement défini par la prise en vue instantanée que nous avons de notre temporalité la plus propre, de notre passe et de notre futur authentiques, au moment de la résolution pour soi-même (SZ, p. 338). Plutôt que d'un véritable présent, il s'agit donc de l'instant critique où saisissant d'un clin d'œil notre propre situation d'être-au-monde, nous sommes appelés à nous décider entre une attitude authentique et inauthentique à propos de notre véritable condition. 
Or cette décision est à ce point tranchante que l'on voit décidément mal comment une présence mondaine pourrait encore y jouer un rôle quelconque. À l'instant de la résolution pour soi-même, aucune intrusion du monde ou des étants mondains n'est possible, car la clairvoyance existentiale requiert précisément l'effacement du monde et, avec lui, la dissipation toutes les illusions de la vie quotidienne déchéante. Il est, en ce sens, légitime de dire que l'analytique existentiale de Sein und Zeit est une ontologie de l'existence sans présent. Cette ontologie nous mène dans l'isolement de ce que Heidegger appelle lui-même le «solipsisme existential» (SZ, p. 188). Celui-ci ne nous donne à considérer que la temporalité d'une existence projetée de son passé authentique en direction du futur authentique de sa mort. Entre ces deux pôles de la trajectoire temporelle de l'existence, la présence des choses et des autres n'a aucune consistance, si ce n'est de nous faire perdre de vue cette temporalité authentique et de susciter ainsi la compréhension inauthentique de notre êtreau-monde.

Tâchons dès lors de conclure à propos de Sein und Zeit. D'abord, rappelons-nous que, dans cet ouvrage, Heidegger conçoit la question du sens authentique de l'être-au-monde de l'homme comme la question première sur le fondement de laquelle il devient possible d'élucider la question essentielle et finale concernant le sens de l'être en général. C'est bien pourquoi l'ouvrage de 1927 se présente comme une ontologie fondamentale. Quels sont maintenant les résultats de l'analyse existentiale de l'être-là humain ? Sans doute peut-on répondre à cela de la manière suivante : lorsque notre être-là est résolu pour soi-même, la compréhension authentique de son existence le porte à considérer sa propre différence ontologique par rapport à l'ensemble des choses dans le monde environnant. Les choses, y compris les autres, sont simplement là comme présentes sous la main sur le mode de la Vorhandenheit. Mais l'homme qui est parvenu à atteindre la compréhension authentique de lui-même sait au contraire que son existence est marquée par une finitude radicale, car elle est ourlée par le néant. Cet homme sait qu'il n'a pas toujours été là, mais que, issu du néant, il a été jeté dans le monde. Il sait du reste qu'il ne sera pas toujours là, mais que son existence est promise au néant de sa propre mort. Certes, entre ces deux extrémités qui ensemble forment l'horizon temporel de son existence, l'homme peut s'attacher au présent, il peut commercer avec les choses et les autres, il peut s'investir dans le monde, et c'est d'ailleurs ce qu'il fait quotidiennement. Mais, au fond, cet engagement quotidien dans le monde de la présence ne saurait donner le change, ni le tromper à propos de ce qu'il a de plus propre. Si tel était le cas,, alors cet engagement serait le signe d'une fuite devant la véritable condition de l'existence. Cette condition est d'avoir été jeté dans un monde où le seul et 
véritable projet de notre être-là est d'y mourir. Aussi l'essence de l'être-aumonde de l'homme est-elle seulement constituée par une temporalité radicalement marquée par la finitude. Cette temporalité est aussi ce qui éclaire l'histoire du Dasein. Mais cette histoire n'est pas à comprendre comme la succession des expériences humaines dans le monde et c'est pourquoi Heidegger préfère parler de l'historialité de l'homme. La différence entre histoire et historialité est simplement que la seconde est déjà écrite, puisqu'elle équivaut au destin inéluctable de l'homme dans un monde où il a été jeté et où sa seule vocation est la mort.

Or, demandera-t-on, si tel est le sens de l'existence humaine sur le fondement de laquelle il s'agit donc de penser le sens de l'être - ainsi que l'indique bien le projet heideggérien d'une ontologie fondamentale - , qu'en est-il dès lors de l'être lui-même et de son sens ? À cette question on ne trouve pas de réponse dans Sein und Zeit. L'ouvrage conclut par la mise en évidence de la temporalité comme sens de l'existence humaine, mais n'envisage pas la question du sens de l'être à partir de cette temporalité. En ce sens, Sein und Zeit est bel et bien un ouvrage inachevé. Dans le plan originaire que l'on trouve à la fin de l'introduction, Heidegger avait conçu son livre en deux parties.

La première portait sur l'interprétation du Dasein à partir de sa temporalité et posait le temps comme horizon transcendantal de la question de l'être. Heidegger l'avait divisée en trois sections :

1. L'analyse fondamentale préparatoire du Dasein

2. Le Dasein et la temporalité

3. Temps et être

La seconde partie prévoyait ensuite une critique ou une déconstruction de la métaphysique à travers sa conception du temps. Elle aussi devait se diviser en trois sections :

1. Kant et le problème du temps

2. Descartes et l'héritage de la philosophie médiévale

3. Le traité d'Aristote sur le temps et les limites de l'ontologie antique

Seules les deux premières sections de la première partie furent publiées sous le titre de Sein und Zeit. La deuxième partie consacrée à une déconstruction de la tradition est très largement compensée aujourd'hui grâce à l'édition des Marburger Vorlesungen, c'est-à-dire des leçons que Heidegger donna entre les années 1924 <et> 1928. Mais ce qui manque absolument est la 
troisième section de la première partie : « Temps et être » dont rien ne nous est dit non plus dans les leçons de Marbourg.

Pourtant, comme le montre le projet même d'ontologie fondamentale de Heidegger, nous sommes induits à penser qu'il devait s'agir d'y montrer comment le caractère temporel de l'existence humaine signifie une certaine temporalité de l'être lui-même, même si de cette temporalité là, justement, rien n'est spécifié dans Sein und Zeit. En outre, l'idée d'une temporalité de l'être devait justifier aussi que, à l'inverse de la thèse ontologique traditionnelle, le sens de l'être n'est pas simplement réductible à la présence ou que « être » ne veut simplement pas dire "être présent». Mais cela semble indiquer alors qu'une certaine dimension d'absence doit aussi appartenir au sens de l'être. Or, si l'absence rentre dans l'acception de l'être, elle ne doit plus avoir seulement une connotation négative au sens où l'on se contente de dire : l'être n'est pas présent ou n'est rien parmi ce qui est présent. Comment dès lors penser positivement cette absence qui entre dans le sens temporel de l'être ? En d'autres termes, comment penser positivement la différence ontologique ?

Sans doute peut-on dire que toute la suite de l'itinéraire de pensée de Heidegger est guidée par cette unique question. Arrivé presque au terme de cet itinéraire, Heidegger prononcera le 31 janvier 1962 à l'université de Fribourg en Breisgau une conférence qui a précisément pour titre Zeit und Sein. Elle vient donc manifestement combler la lacune de Sein und Zeit, puisque son titre coïncide avec celui de la troisième partie de la première division seulement annoncée dans l'ouvrage de 1927.

\section{Le tournant linguistique de Heidegger}

De quoi est-il donc centralement question dans la conférence de 1962 ? Ce texte est assez difficile d'accès, si on ne dispose pas d'une maîtrise suffisante des concepts essentiels qui ont jalonné l'itinéraire de pensée de Heidegger depuis 1927 jusqu'à l'époque de cette conférence. On peut toutefois en résumer le propos essentiel de la manière suivante. La conférence s'ouvre par cette question : qu'est-ce qui autorise à nommer ensemble temps et être ? Être, stipule Heidegger, veut dire depuis toujours la même chose que ce que veut dire en allemand Anwesen. Comme nous l'avons vu dans les analyses faites à l'époque de Marbourg, dans le terme d'Anwesen parle essentiellement le présent. Aussi Anwesen est-il une des façons les plus primitives de dire ce qui est tout autour de nous. Mais, si l'on en élargit la signification, le présent n'est lui-même qu'une dimension qui, avec le futur et le passé, caractérise ce qu'on appelle le temps. L'être en tant qu'Anwesen est donc aussi déterminé par ce 
que veut dire le temps. Mais, en l'occurrence, que veut dire le temps ? Poser cette question revient à interroger plus le sens du terme Anwesen et donc ce que veut dire aussi présence. Le temps, répond Heidegger, n'est pas rien et s'il n'est pas rien c'est tout simplement qu'il y a le temps. Mais que veut dire cet « il y a », lorsqu'on dit qu'il y a le temps ? De toute évidence cet « il y a », ne veut pas dire que le temps est présent, comme lorsque nous disons qu'il y a une table ou qu'il y a une chaise, en voulant dire par là que la chaise et la table sont présentes. Il serait évidemment absurde de dire que le temps est présent de la même façon que le sont la chaise et la table. Autrement dit, le «il y a » en parlant du temps, signifie non pas une présence, mais tout autre chose qu'on peut appeler une « donation ». Précisément la traduction allemande pour « il y a » est « es gibt » qui peut littéralement être entendu comme « ça donne ». Dire donc "qu'il y a le temps» est une manière de dire que "le temps donne ». Mais, si, selon Heidegger, tel est le sens du « il y a » qui prévaut lorsque nous parlons du temps, alors se dresse aussitôt l'autre question de savoir «que donne le temps ? ». Quelle sorte de donné nous procure le temps ? À quoi la réponse de Heidegger est simplement celle-ci : le temps donne lieu, il laisse être ce qui est, il laisse s'ouvrir une présence, bref il donne lieu à ce qui est. Mais dans cette donation ou cette ouverture, le temps lui-même ne se confond donc pas avec la présence qu'il laisse être. Dans sa donation, le temps luimême se tient en retrait. Bien qu'il donne lieu à une présence, le temps luimême se refuse à la présence et ne saurait être confondu avec elle.

$\mathrm{Si}$, comme le dit Heidegger, le temps détermine l'être et si on peut donc les nommer ensemble, alors on voit que comprendre et traduire Anwesen simplement par présence n'est pas correct. Heidegger se propose alors de fournir à Anwesen une autre étymologie consistant à décomposer le terme. Nous obtenons alors " être » pour "Wesen » et un mouvement d'approche pour la particule allemande «an ». Le sens de l'être comme temps signifierait dès lors une approche ou une avancée qui consiste en une donation, qui donc prodigue, mais qui, en même temps, ne se donne pas elle-même dans ce qu'elle prodigue. En d'autres mots, le temps, comme l'être, est une donation qui se retient, se retire ou se dérobe en ce qu'elle donne. Elle se soustrait à ce qu'elle prodigue. Bref, l'être, comme le temps, se fait absent dans le donné ou la présence qu'elle ne fait qu'ouvrir.

Dire l'être comme temps, c'est donc essentiellement dire l'être comme donation et cette donation, on le voit, ne se confond pas avec ce qui est donné. La donation semble plutôt dire ici la vérité qui sous-tend ce qui est donné et contrairement à ce que la tradition a toujours enseigné cette vérité n'indique pas une présence absolue et pleinement dévoilée. Il s'agit au contraire d'une vérité pétrie de réserve ou de retenue. Selon la lecture de Heidegger, c'est bien 
ce que devait signifier originairement le terme grec servant à dire la vérité, à savoir le terme alètheia avant que la métaphysique platonicienne et aristotélicienne ne s'en empare, puisque, ce mot montre que la réserve ou la lèthè reste à l'œuvre dans le sens véritable de la vérité.

Pour désigner cette avance ou cette advenue de l'être qui fait sa vérité, son dévoilement ou, si l'on préfère, son mode de phénoménalisation, Heidegger va introduire, à partir du milieu des années 40 , le verbe sich ereignen (qui signifie arriver, se produire ou au sens transitif : produire, instituer). Plus que vraisemblablement, ce terme fait son entrée dans la Lettre sur l'Humanisme (1946). Par la suite, le substantif Ereignis sera retenu par Heidegger pour désigner le sens de l'être en tant que tel. C'est dans le texte publié en 1957 sous le titre Identité et différence, que l'on trouve tous les éclaircissement nécessaires à la compréhension de l'usage technique de ce terme dans la philosophie de Heidegger.

Dans ce texte, il est question de penser la coappartenance ou l'identité de l'être et de la pensée et d'en revenir donc à la fameuse indication de Parménide que nous avons déjà évoquée : to gar auto estin noein te kai einai. Or, explique Heidegger, cette coappartenance ne se laisse penser, aujourd'hui, qu'à partir du sens que revêt désormais pour nous le mot être. Ce sens est précisément l'Ereignis. À quoi Heidegger donne l'explication suivante : notre époque est celle de la technique, elle corrobore l'âge de la fin de la métaphysique et c'est à cet âge que l'être devient enfin compréhensible comme Ereignis. Pour déterminer l'âge de la technologie en sa véritable essence, Heidegger utilise le terme Gestell que l'on peut traduire, comme nous allons le préciser, par « encadrement » et il écrit : «Dans l'encadrement (Gestell), nous percevons un premier et insistant éclair de l'événement (Ereignis) » (Questions I, <Paris, Gallimard, 1968,> trad. <d'A. Préau> corrigée, p. 272).

Afin de comprendre cette phrase, sans doute est-il besoin d'abord d'éclairer le rapport que Heidegger établit entre notre âge technologique et ce qu'il appelle le Gestell. Il doit s'agir ensuite d'expliquer le lien qui peut exister entre le Gestell et l'être comme événement. Ainsi pourrons-nous enfin comprendre le sens de la coappartenance de la pensée et de l'être telle que Heidegger la conçoit.

Commençons par le premier point: pourquoi l'essence de notre âge technologique peut-elle être saisie à travers le Gestell ? Comme nous l'avons vu dans la première partie de ce cours, selon Heidegger, la fin de la métaphysique est la situation à laquelle conduit la philosophie de Nietzsche. Dans cette philosophie, tous les principes métaphysiques pour assurer et maintenir la présence des étants se dissolvent dans la seule volonté de puissance. Comprendre l'être en termes de volonté de puissance signifie que 
la vérité de l'étant réfère seulement à ce que la volonté de puissance entend faire de l'étant. Autrement dit, l'être de ce qui est en général se réduit à l'estimation qui est faite des choses; l'être n'est plus que l'appréciation ou l'évaluation qui dépend de la volonté de puissance, laquelle, en dehors d'ellemême, ne reconnaît aucune valeur en soi, aucun fondement, aucune vérité, aucun principe de permanence, aucune présence absolue. Si l'on y regarde de plus près, la philosophie de Nietzsche ne mène pas seulement la métaphysique au point le plus extrême de son nihilisme, mais elle est aussi cette pensée avec laquelle s'annonce la véritable essence de l'âge technologique contemporain.

Quelle est, en effet, l'essence de la technique dans laquelle Heidegger voit le trait le plus caractéristique du temps présent ? En 1953, Heidegger a prononcé une célèbre conférence à Munich précisément consacrée à $L a$ question de la technique et où il en discerne le caractère principal, ou l'essence, dans ce qu'il nomme le Gestell. Le terme n'est pas facile à traduire et, bien souvent, les commentateurs préfèrent d'ailleurs ne pas le traduire. En allemand courant, Gestell se dit de tout qui relève des montages ou des assemblages, par exemple une étagère, un tréteau ou un châssis. Manifestement, par-delà toutes ces significations du terme Gestell, Heidegger porte l'accent sur l'activité de rassemblement que toujours présuppose un assemblage quelconque et que marque précisément le préfixe "Ge » dans Gestell. Mais, outre l'action de rassemblement, parle aussi en ce terme l'action de poser, de mettre ou de commettre et qui, cette fois, est marquée par le radical «Stellen ». Sur la base de cette étymologie, ce qui caractérise la technologie serait donc de rassembler l'étant en général tout en le posant ou lui intimant de se présenter tel un fonds disponible pour les opérations de la technique elle-même. Pour le dire d'un seul mot, la technique aurait ainsi pour essence l'encadrement par lequel elle rassemble tout ce qui est en mettant cette totalité en demeure de se conformer au cadre même de la technique. L'effet de cet encadrement sur les choses est qu'elles ne semblent plus dès lors avoir d'essence particulière en dehors du fait d'être posées comme quelque chose d'exploitable, de disponible et d'utilisable, précisément dans le cadre de la technique. Autrement dit, par la technique et conformément à son essence, l'étant en général n'a plus d'autre consistance que de se conformer à l'encadrement auquel le soumet la technique qui affiche ainsi la puissance de sa mainmise sur l'ensemble de $<$ ce $>$ qui est. Ce qui est ne prend valeur qu'en regard des évaluations qui relèvent du plan ou du cadre technologique. Le véritable visage de la technique ressemble donc bien à ce que Nietzsche appelait la volonté de puissance, si ce n'est qu'il ne s'agit certainement pas d'y voir, avec Nietzsche, quelque chose comme la positivité même de l'être, mais d'y reconnaître au contraire une 
négativité ontologique radicale avec laquelle s'anéantissent une à une les différentes manières envisagées, jusque-là, par la tradition pour dire l'être.

Avec l'encadrement par la technique, s'effacent bien toutes les significations métaphysiques qui servaient à dire l'être de l'étant comme principe ou fondement de toute présence. L'époque de la technique est celle où, à propos de l'être de l'étant en général, il n'est plus rien qui puisse encore se laisser dire selon les catégories utilisées par la tradition philosophique. L'homme luimême n'apparaît plus comme le sujet ou le fondement rationnel qui pourrait servir de principe aux étants ou aux objets en général. Cette fonction ontologique, à travers laquelle la subjectivité humaine pouvait affirmer, dans le passé, sa propre liberté en même temps que sa primauté sur le reste des choses, a elle aussi disparu avec le règne de la technique et la généralisation de son encadrement. Ainsi que Heidegger l'explique dans sa conférence de 1953, quoi que les apparences puissent laisser croire, l'homme n'est pas le bénéficiaire de la technique, ni celle-ci un simple instrument de la volonté de puissance humaine. L'homme lui-même est assujetti à l'encadrement technicien ; il appartient à l'avènement de la technique et n'est ce qu'il est que pour autant qu'il s'inscrit dans son cadre en devenant ainsi l'instrument d'une volonté de puissance qui le dépasse. Mais, il reste vrai que, de son côté, l'encadrement technique a besoin de l'homme pour étendre et assurer sa mainmise sur l'ensemble de l'étant.

Pour Heidegger, deux choses essentielles doivent être retenues d'une analyse de ce qu'est l'essence de la technique et ce sont précisément ces deux choses qui, ensemble, forment le contenu de la notion d'Ereignis.

Tout d'abord, à l'époque de la technique, le sens de l'être ne se laisse plus dire à la façon de la métaphysique traditionnelle. Nous ne pouvons plus comprendre son sens en nous aidant des différents concepts qui servaient à exprimer la présence de ce qui est. Plus simplement dit, l'être ne se laisse plus penser comme présence à partir de ce qui est présent car, avec la technique, ce n'est plus la présence des choses et du monde en général qui s'impose, mais seulement un cadre identique où tout se rassemble jusqu'à se confondre dans l'uniformité d'un même fonds ou d'une même mise à disposition. L'être n'a donc plus pour nous le sens d'être présent. Et c'est pourquoi les mots venant à nous manquer pour signifier ce qui est en général, nous sommes plutôt enclin à comprendre l'être de façon beaucoup plus banale comme ce qui a lieu ou ce qui se produit, ou tout simplement comme événement. Événement, tel est justement le premier sens, le sens le plus littéral aussi, du terme Ereignis.

Mais la réflexion sur l'essence de la technique met aussi en lumière le lien de coappartenance existant entre l'homme et l'encadrement technicien. 
D'une part, en effet, en ce qu'il a de propre, l'homme dépend de l'être survenant aujourd'hui sous les traits de cet encadrement. Alors que, d'autre part, l'être que la technique réduit à l'événement, revendique l'homme comme son instrument. Dans l'essence de la technique comme encadrement apparaît donc clairement cette étrange interdépendance moyennant laquelle l'homme est revendiqué par l'encadrement technicien et sommé par lui de rassembler l'étant en un fonds sur lequel peut s'exercer l'emprise de la technique, tandis que l'être ne se produit sous les traits de l'encadrement qu'à travers l'homme.

Pour Heidegger, ici se trouve ce second sens du terme d'Ereignis. En lui parle aussi quelque chose comme le propre (eigen) au sens de l'appartenance ou, mieux encore quelque chose comme un acte d'appropriation (aneignen). On peut, en l'occurrence, comprendre ici une appropriation ou une revendication de l'homme par l'encadrement technicien, comme si la technique - et, sous son emprise, l'événement auquel désormais se réduit ce qui est en général - ne pouvait avoir lieu qu'à travers l'homme, à travers son propre faire, ses propres opérations et ses propres planifications. Mais, si l'être s'approprie ainsi l'homme comme son instrument, de manière réciproque, l'homme s'approprie en quelque sorte la technique, non pas au sens où il la tournerait complètement à son avantage, mais seulement au sens où l'encadrement technicien semble faire désormais le propre de son être-là. Ainsi, à l'âge de la technique, pour peu qu'il s'y applique en pensée, l'homme est en mesure de comprendre que le sens de son propre projet dans le monde lui vient de l'essence de la technique elle-même ou, ce qui revient au même, <qu'il $>$ lui est donné de saisir que son existence tient sa propre condition de ce <que> veut dire être en général sous le jour de l'encadrement.

Cela toutefois, ne devrait surtout pas induire à penser que cette compréhension du propre de l'existence humaine et de son projet représente un dépassement ou une rédemption de la déchéance de la compréhension ordinaire de soi. Au contraire, la déchéance et l'inauthenticité sont ici à leur comble, puisque la double appropriation de l'homme par la technique et de la technique par l'homme mène la compréhension de soi au désarroi le plus total où, tout autant que les choses qu'il n'est pas, l'homme se confond avec elle pour admettre qu'il n'y a plus aucune forme d'essence, de quiddité ou de qualité, et donc aussi de vérité, qui puisse le définir en propre en dehors de la volonté d'encadrement à laquelle il adhère par tout ce qu'il entreprend. En ce sens, l'âge de la technique reste, pour Heidegger, l'âge du péril par excellence, puisque, avec lui, l'être n'est plus dicible à travers aucune vérité et l'homme lui-même, englouti dans cet extrême oubli de l'être, semble perdre tout rapport à l'essence de la vérité en général. Mais, ajoute Heidegger : 
Nous regardons le danger et dans ce regard nous percevons la croissance de ce qui sauve (La question de la technique, trad. $<\mathrm{A}$. Préau, $>$ in Essais et conférences, $<$ Paris, Gallimard, 1958,> p. 45).

Et Heidegger de citer ensuite ces mots de Hölderlin comme pour justifier toute l'attention qu'il nous faut prêter à l'extrême péril que constitue l'âge de la technique :

Là où est le danger, croît aussi ce qui sauve (Wo aber Gefahr ist, wächst das Rettende auch) (Essais et conférences, p. 47).

Ce que Heidegger veut sans aucun doute laisser entendre ici est qu'en ce qu'il est l'ultime étape de la métaphysique qui pousse jusqu'à son extrême accomplissement l'oubli de l'être, l'âge de la technique n'est pas seulement celui du plus total désarroi, mais aussi celui où le désarroi laisse ouverte une fracture suffisante pour que nous parvienne une éclaircie qui, au lieu de l'oubli de l'être, laisse entrevoir au contraire la possibilité d'une mémoire de l'être. Comme le laisse donc entendre Heidegger dans un petit recueil publié en 1957 sous le titre Identität und Différenz, l'essence de la technique comme encadrement rend possible le " pas en arrière », en allemand le Schritt zurück, qui nous fait sortir de la métaphysique, pour penser ce qu'elle n'a jamais pensé : la différence entre l'être et l'étant ou entre l'être et la présence. Mais cette opportunité qui est offerte à la pensée nécessite encore de dépasser l'essence de la technique en ce qu'elle dissimule par son oubli, pour tâcher de penser plus originairement ce qu'elle annonce pourtant : le caractère événementiel de l'être et, conjointement à lui, l'appropriation de l'être-là humain par l'être, comme de l'être par l'être-là humain, soit l'Ereignis.

Mais que veut dire penser plus originairement l'Ereignis par-delà le Gestell, c'est-à-dire penser de façon plus originaire, d'une part, le caractère événementiel de l'être et, d'autre part, la co-appropriation de l'homme et de l'être?

Concernant le premier aspect de la question, ce que nous avons dit à propos de la conférence de 1962, nous a déjà indiqué la voie pour approfondir l'idée d'Ereignis. Dire que l'être est donation ou dire qu'il est événement peut être entendu comme voulant dire une même chose. Mais penser la donation et l'événement en même temps, cela veut plus précisément dire que l'être a sa propre histoire ou, mieux, qu'il est une histoire. En d'autres mots, la manifestation ou l'ouverture de l'être est historique ou historiale au sens où l'être a pour mode de donation d'ouvrir des époques qui précisément font l'histoire de l'être ou la Seinsgeschichte. 
Comme l'indique Heidegger lui-même dans un texte sur Anaximandre repris dans les Holzwege de 1950, le mot « époque », en parlant de l'être ou des époques de l'être, a exactement le sens que les premiers stoïciens donnaient au terme épokhè qui, pour eux, voulait dire le fait de faire halte, de se réserver et donc la retenue ou le retrait. Pour Heidegger qui reprend cet usage, parler du l'événement ou de l'avènement de l'être comme d'un « mode de donation époquale » veut dire que cet événement consiste dans l'ouverture d'une suite d'époques différentes par lesquelles l'être laisse émerger des mondes, tout en se contenant ou en se réservant lui-même dans cette ouverture. Ainsi, peut-on dire que l'être se tient en dehors de sa propre donation et que, se faisant ainsi oublier en ce qu'il donne, il se réserve lui-même en vue d'autres nouvelles donations. Le retrait de l'être peut être dès lors compris comme le moteur même de ses ouvertures successives en des époques qui font tout simplement ce qu'on appelle l'histoire du monde. Pour Heidegger, cette histoire ne saurait être comprise en dehors de la vérité de l'être qui est toujours faite autant de dévoilement que de voilement, comme si la nuit de l'oubli était nécessaire pour que puisse pointer l'aube d'un jour nouveau, la venue d'un nouveau monde ou d'une nouvelle époque.

De l'épokhè de l'être provient l'essence époquale de sa destination, en laquelle consiste la véritable Histoire mondiale. Chaque fois que l'être se contient en son destin, il se produit soudain et imprévisiblement un monde... L'essence époquale de l'être appartient au caractère secrètement temporel de l'être et caractérise ainsi l'essence du temps pensé à l'intérieur de l'être. Ce que l'on se représente d'ordinaire sous le nom de temps n'est que le vide d'une pure apparence de temps recueillie sur l'étant compris comme objet (Chemins qui ne mènent nulle part, p. 275).

C'est précisément cette dynamique du retrait ou de l'oubli de l'être lui-même que, selon Heidegger, l'âge de la technique rendrait enfin pensable après les âges de la métaphysique de la présence.

Mais ce qui importe tout autant, sinon davantage, c'est le second versant de la question de l'Ereignis qui, lui, concerne l'appropriation de l'homme. Or, justement, la thèse de l'ouverture et de la donation époquales de l'être permet une interprétation nouvelle de la facticité de l'homme comme être-au-monde. Dans les termes de Sein und Zeit, cette facticité était comprise comme l'êtrejeté ou la Geworfenheit de l'homme. La même idée se prête désormais à une interprétation plus approfondie, si l'on précise que l'homme est toujours jeté dans une modalité historiale de l'être et qu'ainsi il est toujours inscrit dans une époque de l'être. 
Or, si l'on tient compte du sens complet de l'Ereignis, alors il faut ajouter que l'homme ne se contente pas d'être situé dans une époque de l'être, mais qu'il participe lui-même, en quelque façon, de cette ouverture époquale à travers laquelle l'être ouvre un monde et, pour cette ouverture, requiert l'homme et se l'approprie. C'est dire donc que l'Ereignis ou l'événement époqual de l'être ne se passe pas sans que l'homme lui-même prenne part à cet événement. Plus exactement dit encore, l'homme n'est pas simplement jeté là comme le sont les choses, mas son être-jeté le plus propre le situe dans une époque de l'être où il est entièrement requis par l'être lui-même, de façon telle que son être authentique est d'être-là en tant que ce « là » signifie désormais le lieu de l'événement de l'être. Mais la question est dès lors de savoir par laquelle de ses activités l'homme pourrait ainsi manifester l'essence authentique que lui attribue Heidegger en en faisant le lieu ou le là, de l'Ereignis?

La réponse de Heidegger à cette question désigne clairement l'œuvre d'art. Parmi toutes les activités humaines, c'est dans l'œuvre d'art que Heidegger dit découvrir le produit d'une activité humaine qui n'est pas simplement ontique, ou concernée seulement par les étants, mais qui est à proprement parler ontologique, parce qu'elle a distinctement quelque chose de la nature de l'ouverture époquale de l'être en laquelle il est donné seulement aux étants d'apparaître.

C'est dans le texte de 1935 sur L'Origine de l'œuvre d'art que Heidegger a tenté de spécifier, pour la première fois, ce qui distingue l'œuvre d'art de toutes les autres productions humaines.

En voici la thèse. De façon générale, explique-t-il, les productions humaines consistent dans la fabrication d'objets qui seront intégrables au champ des étants disponibles et utilisables tout autour de nous dans le monde environnant. Pour dire ces choses intramondaines, utilisables, Heidegger se servait, dans Sein und Zeit, du terme allemand de Zeug qui veut simplement dire l'instrument ou l'outil, c'est-à-dire ce qui s'offre à une utilisation quelconque. Comme tel, l'outil n'attire pas l'attention sur lui, tant du moins qu'il fonctionne correctement. Ce n'est que lorsqu'il se casse, devient défectueux ou tout simplement absent qu'il s'impose à notre attention le temps qu'il faut pour le rendre réutilisable ou le retrouver. Quand cela sera fait, l'outil réintégrera le contexte intramondain pour se fondre en lui.

Au contraire, l'œuvre d'art attire toujours l'attention sur elle, parce que, contrairement à l'outil, elle ne se laisse pas rencontrer dans le monde comme un objet parmi d'autres. Autrement dit, l'œuvre d'art ne se fond pas dans le monde environnant des choses utilisables qui sont simplement présentes à portée de main. À l'inverse de ces étants qui sont tellement familiers que nous 
sommes rarement surpris par eux, l'œuvre d'art nous interpelle toujours par sa radicale nouveauté. C'est que, au lieu de se fondre dans le monde qui est déjà là, l'œuvre d'art porte plutôt son propre monde avec elle. Plus exactement, elle laisse apparaitre un monde nouveau en apportant un autre point de vue sur le monde que nous pensions si bien connaître. Comme l'explique Heidegger, c'est aussi pour cette raison que l'œuvre d'art n'est pas simplement une perspective nouvelle projetée sur un monde déjà constitué en dehors d'elle. Bien plutôt, l'œuvre d'art nous fait entrer en un monde dont, elle seule, est le projet, un monde qui n'est donc ouvert que par elle. Le caractère essentiel de l'œuvre d'art est donc d'instituer un monde à travers un ensemble de significations nouvelles.

Mais ce qu'il y a de vraiment remarquable dans cette institution de monde, est que, en même temps, l'œuvre d'art se fait toujours aussi comme une réserve de significations qui restent à découvrir. Dans l'œuvre d'art, du sens demeure toujours d'une certaine manière en retrait, en suspens, en entente, ou tout simplement enfoui dans le monde ouvert par l'œuvre d'art ellemême. Aussi est-ce précisément par ce phénomène que Heidegger cherche à faire ressortir le caractère proprement ontologique de l'œuvre d'art. Celle-ci ouvre un monde selon une modalité très similaire à la propre modalité époquale de l'être. Comme l'événement de l'être, l'institution réalisée par une œuvre d'art est toujours en même temps dévoilement et voilement, mise au jour et dissimulation.

C'est ce que, dans le texte de 1935, Heidegger tente d'exprimer à travers ce qu'il appelle le conflit du «monde et de la terre» dans lequel il voit l'essence même de l'œuvre d'art. Comme nous l'avons dit, un « monde » est ce qui émerge et se dresse telle une édification, grâce à l'œuvre d'art. Mais cette édification ou cette construction a un sol qui la supporte. La terre (Erde) est le nom exploité par Heidegger pour appeler ce sol. Mais en quel sens fautil dire que la terre entre aussi dans la véritable nature de l'œuvre d'art et qu'elle est donc nécessaire à la propre fonction artistique qui est d'ouvrir et d'instituer un monde? De nouveau, comparons avec la signification que revêt la terre dans l'acte ordinaire de manufacturer un objet de façon à le rendre présent et donc disponible. Dans ce simple produit de l'acte manufacturier, la terre n'est que le matériau de la construction et, d'une certaine façon, ce matériau disparaît dès lors que le produit est achevé et qu'il entre dans le champ de la disponibilité. Lorsqu'il est un produit fini et donc prêt à l'usage, le pot, par exemple, a définitivement pris la place de la glaise qui a permis de le fabriquer. Par contre, dans l'œuvre d'art, la terre demeure active tel un composant irréductible. Bien sûr, l'œuvre d'art est aussi fondée sur un matériau qui est transformé par elle: la pierre n'est plus seulement de la pierre dans la 
sculpture, les sons ne sont plus seulement des sons dans la symphonie, les couleurs ne sont plus seulement des couleurs sur le tableau, les mots ne sont plus seulement des mots dans le poème. L'œuvre d'art a fait en sorte que du sens s'empare du matériau pour le transformer de manière à ce que puisse advenir un étant. Mais, pourtant, la terre reste bien là comme un fond inépuisable. Le sculpteur utilise la pierre comme le maçon, mais contrairement à celui-ci il ne parvient pas à l'estomper au profit d'autre chose, par exemple le mur. C'est qui indique que, dans l'œuvre d'art, cette même pierre reste une réserve inépuisable pour d'autres significations qui pourront s'en emparer à leur tour et faire émerger ainsi d'autres mondes. La matière ou la terre sur laquelle se fonde l'œuvre d'art est donc aussi ce qui se tient toujours en réserve par rapport au monde ouvert par cette œuvre et se réserve justement pour l'ouverture d'autres mondes. En ce sens, l'œuvre d'art est donc faite du combat que se livrent le dévoilement et le voilement. Avec l'œuvre d'art et son apport de sens, un monde tend à l'ouverture et au dévoilement, mais en même temps persiste la tendance inverse par laquelle la terre résiste à l'ouverture en retenant le sens par une sorte d'aspiration au voilement. En raison de cette tension, même prise pour elle-même, l'œuvre d'art ne se laisse jamais pénétrer dans tout son contenu de sens : ce qu'elle ouvre comme un monde ne va pas sans tout ce qu'elle referme et qui est à sa propre réserve de sens. Quant à cette réserve elle appartient à la plénitude inépuisable de la terre qui se referme toujours sur soi. Dans le texte de 1935, Heidegger écrit :

\begin{abstract}
Monde et terre sont essentiellement différents l'un de l'autre, et cependant jamais séparés. Le monde se fonde sur la terre, et la terre surgit au travers du monde. Cependant, la relation entre monde et terre ne décrépit point en une vide unité d'opposés qui ne se concernent en rien. Reposant sur la terre, le monde aspire à la dominer. En tant que ce qui s'ouvre, il ne tolère pas d'occlus. La terre, au contraire, aspire, en tant que reprise sauvegardante, à faire enter le monde en elle, à l'y retenir (Chemins qui ne mènent nulle part, p. 37).
\end{abstract}

Du point de vue de Heidegger, l'important est ici de montrer que, si l'œuvre d'art est certes toujours un produit de l'homme, elle est néanmoins bien plus que cela. En effet, de par sa propre structure dévoilante et voilante, l'œuvre d'art est le produit de l'activité humaine où resplendit quelque chose de plus essentiel que l'homme lui-même et qui n'est autre que la vérité de l'être comme événement, avènement, ou Ereignis. Bien sûr, c'est aussi en ce sens que Heidegger peut définir l'œuvre d'art comme «la mise en œuvre de la vérité », das Ins-Werk-Setzen der Wahrheit (Chemins qui ne mènent nulle part, p. 30). 
Dans la conférence de 1935, Heidegger continue son analyse du caractère ontologique de l'œuvre d'art à propos plus particulièrement de la poésie, en définissant celle-ci comme l'essence de tous les arts : «Laissant advenir l'événement de la vérité de l'étant comme tel, tout art est essentiellement poésie » (Chemins qui ne mènent nulle part, p. 56).

Dichtung ou poésie, voilà donc le terme qui, selon Heidegger, dit au mieux la création ou l'institution. Ce qui est donc aussi une manière de rapprocher le concept de création du concept d'acte de parole et ou de langage. Mais, bien sûr, encore faut-il s'entendre sur ce que parole ou langage veut dire. Reprenant la même distinction que celle qu'il avait faite à propos des œuvres humaines afin de dégager la spécificité de l'œuvre d'art, Heidegger distingue la langage comme instrument et le langage comme véritable création. Le langage comme instrument est celui qui ne sert qu'à la communication ou à la conversation à l'intérieur d'un monde déjà constitué. Au contraire, le langage véritablement créatif est celui qui institue un monde, et fait advenir l'étant en général. Comme le dit Heidegger, « là où aucune langue ne se déploie, il n'y a aucune ouverture de l'étant $»$, et, faut-il ajouter, aucun monde ne pourrait advenir.

L'interprétation de l'œuvre d'art comme poésie ou Dichtung finit donc par l'affirmation que le langage est le lieu de l'avènement de l'être, le lieu de son Ereignis. Comme nous l'avons vu, d'une part, l'homme est jeté dans une ouverture époquale de l'être, mais, d'autre part, il est en même temps requis par l'être pour dire cette ouverture et par là-même faire en sorte qu'advienne un monde. En d'autres termes, le langage au sens propre de la Dichtung, n'est rien d'autre que la façon dont l'être advient, la façon dont il se fait événement, se fait Ereignis.

Rappelons-nous le plan de Heidegger dans Sein und Zeit. Son analytique existentiale s'était arrêtée sur la temporalité propre du Dasein en en faisant le fondement qui, pour la première fois, permettait de penser la vérité de l'être autrement que comme présence, mais de penser cette vérité comme temps. Nous pouvons voir maintenant que ce qui n'était encore qu'un projet dans Sein und Zeit va prendre la forme suivante dans les écrits postérieurs de Heidegger : si l'être n'est rien de présent comme le sont les étants tout autour de nous, c'est qu'il a une histoire. L'histoire de l'être est sa vérité. Ce qui veut dire que l'être n'est rien d'autre qu'une donation qui ouvre différentes époques, dans laquelle l'homme se trouve chaque fois engagé. Par là s'offre la possibilité de saisir un autre sens de la Geworfenheit ou de l'être-jeté de l'homme dont il était déjà question dans Sein und Zeit. La façon dont l'homme est jeté dans le monde, et donc ce qu'est sa situation dans le monde, n'est pas toujours la même, car le monde lui-même est chaque fois une ouverture historique de l'être lui-même, 
il est chaque fois une modalité de son avènement ou de son Ereignis. Mais, d'un autre côté, il appartient aussi au sens de l'Ereignis que l'homme qui est jeté dans une époque de l'être ne se contente pas d'y être situé, mais s'y trouve lui-même appelé à participer à l'ouverture du monde. Autrement dit, l'existence de l'homme, son propre être-au-monde, ne se limite pas à être dans une certaine ouverture d'un monde qui serait déjà réalisée, parce que l'homme est aussi appelé à contribuer à cette ouverture.

On se rappelle que, dans Sein und Zeit, Heidegger opposait radicalement l'existence inauthentique de l'homme cherchant à fuir sa condition la plus propre dans le monde ambiant et l'existence authentique seulement déterminée par l'assomption de la condition spécifique de notre être-là. Mais si l'authenticité voulait dire ainsi l'acception ou la résolution pour la temporalité et la finitude propres de notre être-là, l'authenticité n'était pas pour autant déjà pensée dans sa relation positive à l'être lui-même. Elle n'avait au contraire de relation négative à l'être tant que celui-ci était compris comme présence. Il en va désormais tout autrement avec la pensée de l'être comme Ereignis. En effet, l'idée d'avènement laisse ouverte une possibilité pour l'homme d'habiter son monde sans pour autant que cette habitation le mène inévitablement à la déchéance. Parce que la pensée de l'être comme avènement est aussi une façon pour l'homme de comprendre différemment sa propre relation au monde, cette pensée permet de prendre la mesure d'un habiter authentique que l'on ne trouvait pas dans Sein und Zeit. Dans l'ouvrage de 1927, Heidegger opposait partout à la déchéance de l'habitation dans le monde la reconnaissance de notre extranéité (Unheimlichkeit), c'est-à-dire de notre condition la plus propre qui est d'être sans chez-soi (Un-zuhause) dans le monde. Mais avec la pensée de l'être comme Ereignis, une forme d'habitation authentique dans le monde devient pensable et cette forme authentique, Heidegger la nomme désormais poésie (Dichtung). C'est là le véritable tournant ou la Kehre qui est opérée par l'écrit sur L'Origine de l'œuvre d'art. Dans un texte de la même époque intitulé Hölderlin et l'essence de la poésie (1936), Heidegger en exprimera l'idée centrale en reprenant ce vers tardif de Hölderlin : " Dichterisch wohnet der Mensch » : «L'homme habite en poète ».

Certes, y explique Heidegger, la poésie ne se fait pas à partir de rien, elle-même présuppose toujours un langage. Mais quelle en est l'essence ? Le langage n'est-il qu'un moyen qui ne servirait à d'autre fin que l'échange ou la communication? N'est-il qu'un instrument ou un outil, c'est-à-dire quelque chose que nous tiendrions à notre disposition ? Cette approche utilitaire du langage fait perdre de vue qu'il est bien plus que cela, car il a le pouvoir d'instituer un monde en nommant les choses. Cette nomination ne veut pas dire simplement la capacité de mettre un nom sur ce qui est, car c'est par la 
nomination que les choses deviennent ce qu'elle sont. Ici se trouve donc le véritable pouvoir créatif du langage qui, en donnant leur sens aux étants en général, fait qu'un monde s'ouvre. Le terme " poésie » n'a d'autre sens que d'exprimer ce pouvoir qui est le propre du langage. Comme le dit Heidegger :

Le poète nomme les dieux et nomme toutes choses en ce qu'elles sont. Cette nomination ne consiste pas à pourvoir simplement d'un nom une chose qui auparavant aurait été déjà bien connue ; mais le poète disant la parole essentielle, c'est alors seulement que l'étant se trouve par cette nomination nommé à ce qu'il est, et ainsi connu comme étant. La poésie est fondation de l'être par la parole... Mais en même temps que les dieux sont originellement nommés et que l'essence des choses passe à la parole, pour que les choses commencent alors de luire, en même temps que cela s'historialise, l'être-là de l'homme accède à une relation ferme et est assis sur une base. Le dire du poète est fondation non seulement au sens d'une libre donation, mais également en ce sens qu'il assied et assure sur sa base l'être-là de l'homme (Approche de Hölderlin, trad. $<$ H. Corbin, M. Deguy, F. Fédier, J. Launay, Paris, Gallimard $>$, p. 52-53).

Or, si un monde n'advient qu'avec un langage, cela veut dire que le monde s'historialise car le langage lui-même est historialisé. Mais que signifie cette autre caractéristique du langage ? Pour Heidegger, la réponse n'est pas à chercher ailleurs que dans ce vers de Hölderlin qui dit : "Nous sommes un dialogue (Gespräch) ». À moins de retomber dans la dimension du langage comme instrument que nous avons dû écarter pour en saisir le véritable pouvoir ontologique, le dialogue ne signifie pas ici notre capacité de communication, mais il désigne plutôt l'unité sur la base de laquelle il devient possible de s'unir dans la communication. Autrement dit, il n'y a de dialogue que parce que notre parole reste relative à cet un. Quant à cette unité ou $<$ à $>$ cet identique qui nous habilite à parler et à nous entendre à propos des mêmes choses, il ne peut concerner que le monde tel que le temps le laisse être, ou encore le monde tel qu'il s'historialise. D'où, comme le dit Heidegger :

Être-un-dialogue et être dans l'histoire, ces deux choses sont d'ancienneté égale... elles forment une seule et même chose (Approche de Hölderlin, trad., p. 50).

Être dans l'histoire dit bien ce qu'il en est de la situation de notre être-là en tant qu'il nomme les choses et, par là, fait advenir un monde par son langage en même temps que ce langage forme l'événement fondamental de notre l'êtrelà. Ce que Heidegger résume de la façon suivante : 
Le langage n'est pas seulement un instrument que l'homme possède à côté de beaucoup d'autres ; le langage est ce qui, en général et avant tout, garantit la possibilité de se trouver au milieu de l'ouverture de l'étant. Là seulement où il y a langage, il y a un monde, c'est-à-dire ce cercle continuellement changeant de décision et d'entreprise, d'action et de responsabilité, mais aussi d'arbitraire et de tumulte, de déchéance et d'égarement. Et là seulement où il y a un monde, il y a histoire. Le langage est un bien en un sens plus originel. Qu'il soit le bien, caution de ce monde et de cette histoire, cela veut dire qu'il garantit que l'homme puisse être en tant qu'historial. Le langage n'est pas un instrument disponible ; il est, tout au contraire, cet avènement (Ereignis) qui lui-même dispose de la suprême possibilité de l'être de l'homme. C'est de cette essence du langage que nous devons tout d'abord nous assurer, pour concevoir vraiment la sphère d'action de la poésie et la saisir ainsi vraiment elle-même (Approche de Hölderlin, trad., p. 47-48).

Le moment est venu de conclure en demandant comment, selon Heidegger, l'être humain peut être authentiquement être-là. Comment peut-il être en propre le Dasein ? Nous avons eu l'occasion de voir quelle est la réponse de Heidegger à cette question, à partir des années 30. L'homme est authentiquement son propre être-là pour autant qu'il se sait requis par l'être et répond à cette requête. Assumer la requête signifie, pour lui, habiter le monde d'une manière poétique. En d'autres mots, être authentiquement son être-là signifie, pour l'homme, être le lieu où l'être lui-même advient et cet événement ontologique ne peut se produire qu'à travers l'activité poétique; à savoir dans le langage créatif de l'homme. En effet, le propre de la poésie est d'ouvrir un monde en nommant et en signifiant tout ce qui est en ce monde. Mais, cette nomination ne consiste pas à donner un nom à des choses qui seraient déjà connues antérieurement au fait de les nommer. Nommer est plutôt la façon dont les étants en général deviennent ce qu'ils sont. La parole créative de l'homme projette elle-même un monde en l'instituant par un ensemble de significations qui sont nouvelles en comparaison de ce qu'était la conception du monde jusqu'alors.

Cependant, ce n'est pas seulement parce que la poésie est ouverture d'un monde qu'elle est la caractéristique ontologique de l'homme. L'ouverture d'un monde par la créativité du langage indique son pouvoir ontologique surtout par le fait que cette donation est toujours aussi retenue et retrait. Dans toute constitution d'un monde par la signification, se passe toujours quelque chose comme une mise en réserve du sens qui reste en arrière et, d'une certaine façon, se cache dans le monde ouvert par le langage créatif. En d'autres termes, par la production linguistique du sens, un monde tend à se manifester, tandis que le sens lui-même tend à se dérober et à rester en retrait du monde ouvert par le 
sens. Aussi le sens se réserve en lui-même et jamais n'est pleinement ouvert en ce qu'il ouvre. C'est précisément avec cette double tendance au voilement et au dévoilement que Heidegger veut faire ressortir la caractéristique spécifiquement ontologique du langage. Par son pouvoir créatif, le langage ouvre un monde selon cette modalité en laquelle il est assez facile de reconnaître la propre modalité époquale de l'être comme avènement ou Ereignis. Aussi, dans la mesure où il capable d'habiter de manière poétique, l'homme se révèle luimême comme cet étant qui est possédé par l'être en tant qu'avènement. D'un autre côté, l'avènement époqual de l'être comme histoire appelle le langage comme lieu de son propre phénomène à la fois en tant que dévoilement et recouvrement. En d'autres termes, l'avènement de l'être est toujours linguistique, tandis que le langage est la seule dimension ontologique de l'existence humaine.

C'est sur la base de cette thèse que, au début des années 50, Heidegger tentera de clarifier ce que signifie la philosophie. Selon lui, en tant que pensée concernant l'être, l'investigation philosophique doit être conçue comme une écoute du langage dans son pouvoir d'ouverture et de dévoilement. Sur ce point, Heidegger en revient à la notion d'herméneutique qu'il avait exploitée, pour la première fois au début des années 20, au cours des années de Fribourg qui précèdent son enseignement à Marbourg. À cette époque, avec l'herméneutique, la question n'était encore que de comprendre et d'interpréter la facticité de l'existence humaine. À présent, le problème est d'investiguer l'origine du langage. En d'autres mots, le problème est de comprendre l'appel d'où provient le langage. Ainsi, l'herméneutique du langage devient une élucidation du langage au sens littéral d'une Er-örterung, c'est-à-dire d'une tentative de remonter au lieu (Ort) d'où provient le langage pris au sens de son pouvoir authentique. Telle que la comprend donc Heidegger, l'herméneutique cherche à donner à entendre ce dont le langage lui-même est le message ou l'annonce. C'est précisément là le sens que Heidegger attribue à l'herméneutique dans son ouvrage de 1959 intitulé Acheminement vers la parole (Unterwegs zur Sprache). Le premier texte que reprend cet ouvrage est le résumé d'une discussion avec un étudiant japonais à qui Heidegger explique sa propre compréhension de la tâche herméneutique. À ses yeux, le terme « herméneutique » vient étymologiquement du grec "herméneuein » qui signifie : "la révélation qui survient du fait de prêter écoute à une annonce» (dans la mythologie grecque, Hermès lui-même est, comme on sait, le messager des dieux). Cette annonce est celle de l'être en son propre secret. Parce qu'elle ne peut être faite qu'à travers le langage, non dans son contenu, mais dans son essence, l'herméneutique se présente comme une ontologie et cette ontologie doit être comprise comme une herméneutique du langage. Suivant Heidegger, 
l'ontologie prend ainsi un tout nouveau visage par comparaison avec ce qu'elle était dans la conception traditionnelle de la métaphysique. Celle-ci a toujours tenté de saisir le sens de l'être en général à partir du fondement où ce sens semblait s'illuminer. Peu importe que ce fondement ait été compris comme ousia, Dieu ou le sujet humain, il désignait partout le sol à partir duquel devient compréhensible l'être présent des choses en général. Inversement, en se tournant vers l'essence poétique ou créative du langage et portant son attention sur le lieu d'où il provient, l'herméneutique montre que ce lieu n'est <pas> quelque chose de l'ordre du fondement ou du Grund, mais quelque chose comme un sans fond ou un Abgrund, puisque ce qui s'annonce à travers le langage est un fonds inépuisable dont, en ses propres ressources sémantiques, le langage lui-même porte la marque. Ce fonds sans fond est une autre façon d'exprimer la réserve de sens insondable qui sous-tend toute production de sens. Ici, peut-être, se trouve le véritable dénouement du tournant de l'ontologie en une herméneutique du langage.

Parmi tous les continuateurs de la philosophie de Heidegger, HansGeorg Gadamer est sans aucun doute celui qui a su tirer les principales conclusions du tournant à la fois ontologique et herméneutique initié par le premier. Ses conclusions sont clairement exprimées dans la thèse essentielle de son ouvrage majeur de 1960, Vérité et méthode (Wahrheit und Methode) : "L'être qui peut être compris est langage » (Sein, das verstanden werden kann, ist Sprache) ( $W<$ ahrheit und $>M<$ ethode. Grundzüge einer philosophischen Hermeneutik, J.C.B. Mohr (Paul Siebeck), $4^{\mathrm{e}}$ éd., 1975>, p. 450 ; trad. <É. Sacre, Vérité et méthode. Les grandes lignes d'une herméneutique philosophique, Paris, Seuil>, 1976, p. 330). Il s'agissait ici de montrer que cette thèse, qui revient donc à défendre l'idée d'une essence linguistique de l'être, vaut aussi comme conclusion logique de l'ontologie de Heidegger.

\section{Note de l'éditeur}

Robert Brisart (1953-2015) a dispensé le présent cours à l'Université du Luxembourg, où il occupa un poste de professeur associé de 2000 jusqu'à sa mort. Ce cours de vingt heures s'inscrivait dans le cadre d'un Master in European Philosophy. Bien que le manuscrit soit en français, il était exposé en anglais. Toutes mes adjonctions autres que purement orthographiques, grammaticales et typographiques sont entre chevrons simples $(<>)$. (Denis Seron) 